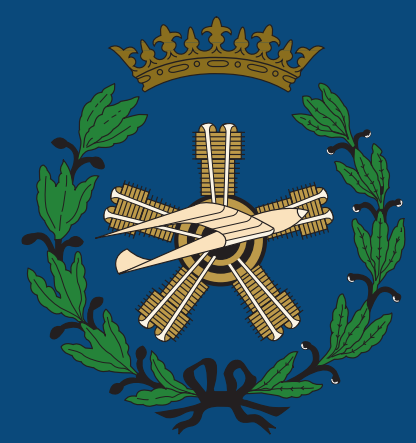

\title{
A fast IMPES multiphase flow solver in porous media for reservoir simulation
}

\author{
Doctoral Thesis \\ By \\ Carlos Redondo Calle \\ Aeronautical Engineer
}

June 2017 



\section{A fast IMPES multiphase flow solver in porous media for reservoir simulation}

by

Carlos Redondo Calle

Aeronautical Engineer

Advisors:

Eusebio Valero Sánchez

Ph.D. in Aeronautical Engineering

Gonzalo Rubio Calzado

Ph.D. in Aeronautical Engineering

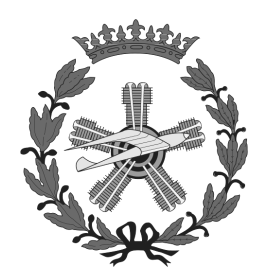

Madrid, June 2017 
This thesis has been partially supported by Repsol.S.A under the research grant P130120150.

Esta tesis ha sido cofinanciada por Repsol.S.A en el marco del proyecto de investigación P130120150. 


\section{POLITÉCNICA}

El tribunal nombrado por el Sr. Rector Magnífico de la Universidad Politécnica de Madrid, el día de de $20 \ldots . .$.

Presidente:

Vocal:

Vocal:

Vocal:

Secretario:

Suplente:

Suplente:

Realizado el acto de defensa y lectura de la Tesis el día de de $20 \ldots$. en la E.T.S.I./Facultad.

Calificación.

EL PRESIDENTE

LOS VOCALES

EL SECRETARIO 



\title{
A fast IMPES multiphase flow solver in porous media for reservoir simulation
}

\begin{abstract}
In this thesis, a methodology to efficiently solve the multiphase flow through a porous media in the near wellbore region is presented. The mathematical models, the numerical methods and practical implementation details for a functional 2D solver are presented.

The methods described in this thesis are the base of an industrial 3D code. Thus, many efforts have been made to validate all the new models to guarantee robustness and stability in the solution without penalising the efficiency.

The numerical discretisation is based on the IMplicit Pressure Explicit Saturation (IMPES) approach. While most of the works in the literature rely on Fully Implicit Methods (FIM) to simulate the reservoir scale, this is not suited, in general, for the near wellbore region, where smaller computational and physical times are required, therefore parametric history matching models are preferred to solve this region. However, parametric models present several uncertainties that can affect significantly the estimation of the pressure drop. An accurate and fast simulation of that region is therefore required.

Here, a novel and robust implementation of the IMPES scheme is presented, aiming to reduce the large computational cost of the (implicit) pressure equations and to increase the robustness and reliability of the scheme. The method takes advantage of the short physical time steps between iterations, imposed by the explicit saturation solver, to produce a new pressure field at the numerical cost of an explicit scheme using an iterative solver. One, two and three-dimensional numerical experiments are carried out to show the validity of the proposed discretisation. It is shown that the cost of the method is reduced an order of magnitude when compared to the classical IMPES, which outperforms, in a lot of scenarios, the state of the art of current implicit methods.
\end{abstract}

Keywords: Porous media, multiphase flow, reservoir simulation, IMPES, iterative methods. 


\section{A fast IMPES multiphase flow solver in porous media for reservoir simulation}

\section{Resumen}

En esta tesis se propone una nueva metodología para resolver, de forma eficiente, el flujo multifásico en medio poroso para la región próxima a las perforaciones en yacimientos de hidrocarburos (near-wellbore). En este trabajo se presentan los modelos matemáticos, los métodos numéricos y detalles prácticos de la implementación para un simulador 2D.

Los métodos descritos en esta tesis han servido como base para un simulador 3D industrial, por lo tanto, los principales esfuerzos se han dedicado a la validación de los nuevos modelos para garantizar la estabilidad y la robustez del método sin que la eficiencia se vea afectada.

La discretización numérica está basada en el método IMPES (Implicit Pressure Explicit Saturation), en el cual, el problema asociado a la presión se trata de forma implícita mientras que el problema asociado a la saturación se resuelve de forma explícita. La mayoría de códigos presentes en la literatura utilizan métodos totalmente implícitos o Fully Implicit Methods (FIM) para la simulación de yacimientos. Estos métodos no son, en general, apropiados para la región cercana al pozo donde se necesitan pasos temporales más reducidos. En esta región se usan modelos paramétricos basados en datos históricos. Estos métodos presentan incertidumbres que pueden afectar de forma significativa a la estimación de la distribución de presión. Por consiguiente, es necesario un método para simular de forma precisa y eficiente el flujo en esta región.

En esta tesis se presenta una implementación novedosa del esquema IMPES. Se reduce el coste computacional asociado a las ecuaciones de presión (parte implícita) y se mantiene la estabilidad del esquema. El método se aprovecha de los pasos temporales reducidos, impuestos por la ecuación de saturación, para actualizar el campo de presión por el coste numérico de una iteración explícita utilizanto un método iterativo. La discretización propuesta se ha validado con con experimentos numéricos en una, dos y tres dimensiones. Se demuestra, además, que el método propuesto reduce en un orden de magnitud el tiempo computacional de las implementaciones clásicas del IMPES y mejora, en numerosos escenarios, el rendimiento de los métodos implícitos.

Keywords: Medio poroso, flujo multifásico, simulación de yacimientos, IMPES, métodos iterativos. 


\section{Acknowledgements}

First, I would like to thank my advisors, Eusebio Valero and Gonzalo Rubio, for their support and encouragement during this thesis. I also would like to thank Ángel Rivero for for the coordination of the project that has led to this thesis and his help and interesting discussions during my time in the Centro de Tecnología de Repsol.

I would like to thank the Applied Mathematics Department for the incredible work environment during the stays at the University.

I also want to thank my family, my friends and especially Araceli, without whom this $\mathrm{PhD}$ would have never been possible.

Finally, I want to thank to Repsol that has funded this doctoral thesis under the research grant $\mathrm{P} 130120150$. 


\section{Agradecimientos}

Primero, me gustaría agradecer a mis tutores de tesis, Eusebio Valero y Gonzalo Rubio por su apoyo y ánimos durante el desarrollo de esta tesis. Me gustaría agradecer también a Ángel Rivero por la coordinación del proyecto que ha dado lugar a esta tesis y por su ayuda e interesantes discusiones durante mis estancias en el Centro de Tecnología de Repsol.

Quiero agradecer también al Departamento de Matemática Aplicada por el fabuloso ambiente de trabajo en mi estancia en la Universidad.

También quiero dar las gracias a mi familia y amigos, en especial a Araceli, sin los cuales este doctorado no habría sido posible.

Por último, agradecer a Repsol por la financiación de esta tesis, en el marco del proyecto de investigación P130120150. 
1 Introduction 1

1.1 The problem of reservoir simulation . . . . . . . . . . . . 1

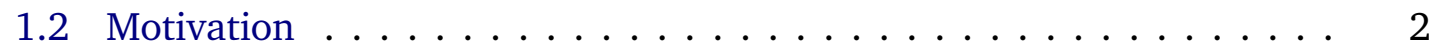

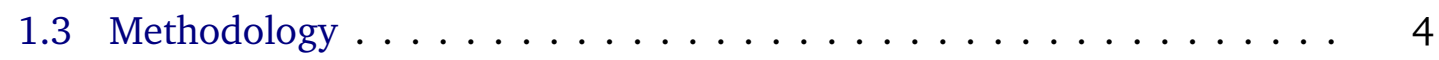

2 Mathematical Models 7

2.1 The porous media . . . . . . . . . . . . . 7

2.1 .1 Continuum approach $\ldots \ldots \ldots \ldots$

2.1 .2 Properties of porous media . . . . . . . . . . . . 9

2.2 Flow in porous media . . . . . . . . . . . . . . . . . . . . . . 11

2.2 .1 Darcy's law . . . . . . . . . . . . . . . . . 11

2.2 .2 Validity of Darcy's law . . . . . . . . . . . . . . . . . . . . . . . . . . . . 13

2.2 .3 Single-phase flow . . . . . . . . . . . . . . . 14

2.3 Multi-phase flow in porous media . . . . . . . . . . . . . . . 15

2.3.1 Immiscible two-phase flow . . . . . . . . . . . . . . 15

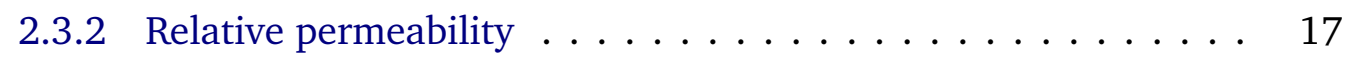

2.3 .3 Capillary pressure . . . . . . . . . . . . . 20

2.3.4 Immiscible incompressible two-phase flow problem . . . . . . 23

3 Numerical Methods $\quad 25$

3.1 Spatial discretisation . . . . . . . . . . . . . . . . . . . . 25

Scheme order . . . . . . . . . . . . . . . . . . . . . . . . . . . . . . . 29

3.1 .1 Boundary conditions . . . . . . . . . . . . . . . . . . . . . . 29

Pressure inflow $/$ outflow . . . . . . . . . . . . . 30

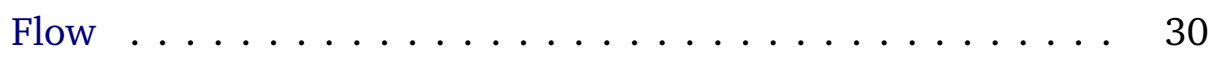

Wells ....................... 30

3.1 .2 Mesh refinement . . . . . . . . . . . . . . . . . . . . . . . . 32

3.2 Temporal discretisation $\ldots \ldots \ldots \ldots \ldots \ldots$

3.3 Iterative pressure solver $\ldots \ldots \ldots \ldots \ldots \ldots \ldots$ 


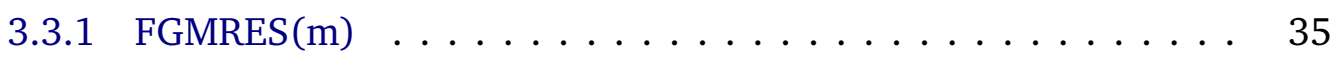

Preconditioners ...................... 36

3.3 .2 Iterative solver tolerance $\ldots \ldots \ldots \ldots \ldots$

4 Numerical Experiments and Validation $\quad 41$

4.1 Validation cases . . . . . . . . . . . . . . . . . . . . 41

4.1.1 Case 1. Buckley-Leverett problem. . . . . . . . . . . . . . . 42

4.1.2 Case 2. SPE10 dataset $1 \ldots \ldots \ldots$. . . . . . . . . . 44

4.1 .3 Case 3. Banded plug . . . . . . . . . . . . . . . 47

4.1.4 Case 4. Heterogeneous plug . . . . . . . . . . . . . . . 49

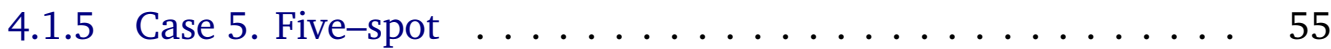

4.1.6 Case 6. SPE10 dataset $2 \ldots \ldots \ldots \ldots \ldots$

4.2 Analysis of iterative solver . . . . . . . . . . . . . . . . 61

4.2 .1 Impact of preconditioner . . . . . . . . . . . . . . . 61

4.2 .2 GMRES restart . . . . . . . . . . . . . . . 63

5 Multiphase 2D IMPES Solver $\quad 65$

5.1 Input/Output files . . . . . . . . . . . . . . . . . . . . . 66

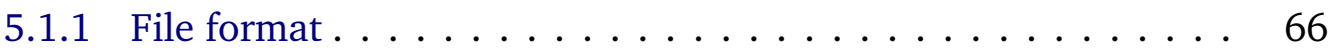

5.2 Implementation methodology $\ldots \ldots \ldots \ldots \ldots$. . . . . . . 67

5.2 .1 Sparse matrix storage . . . . . . . . . . . . . 70

5.2 .2 Parallelisation . . . . . . . . . . . . . . 71

$\begin{array}{ll}\text { Conclusions } & 75\end{array}$

$\begin{array}{ll}\text { Appendices } & 77\end{array}$

$\begin{array}{lll}\text { A Data structure. AOS vs SOA } & 79\end{array}$

$\begin{array}{llr}\text { B Fortran - Python plotting } & 87\end{array}$ 
Acronyms

bbl

CG

CSR Compressed Sparse Storage

EOS Enhaced Oil Recovery

FIM Fully Implicit Methods

GMRES Generalised Minimal Redisual

HPC High Performance Computing

ILU Incomplete LU factorization

IMEX Implicit Explicit Method

IMPES Implicit Pressure Explicit Saturation

LHS Left Hand Side

NZ Non-zero pattern

PV Pore Volume

PVI Pore Volume Injected

REV representative elementary volume

RHS

Right Hand Side
RK

SOR

SI

SSOR

SPE

TVD

VTK
Runge Kutta

Successive Overrelaxation

International System of

Units

Symmetric Successive Overrelaxation

Society of Petroleum Engineers

\section{Greek Symbols}

$\Delta t$

Time step

$\Delta S_{\max }$

Maximun saturation variation per time step

$\varphi$

$\gamma$

$\lambda$

Slope limiter function

Surface tension

Phase mobility

Porosity distribution index

$\mu \quad$ Dynamic viscosity

$v \quad$ Kinematic viscosity

$\phi \quad$ Porosity

$\rho \quad$ Density

$\theta \quad$ Contact angle 


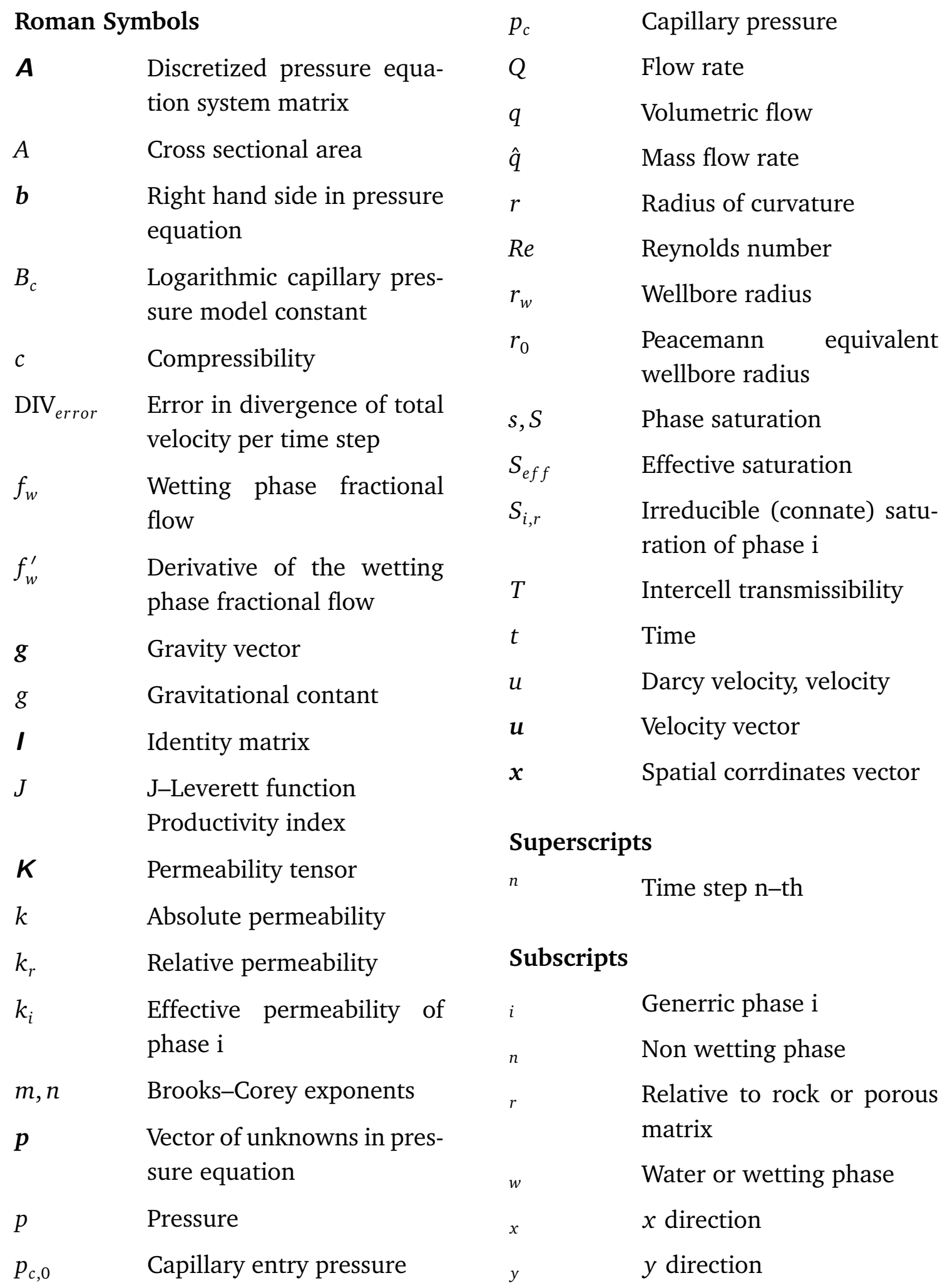




\section{LIST OF FIGURES}

2.1 Schematic of two immiscible phases on porous media at microscopic

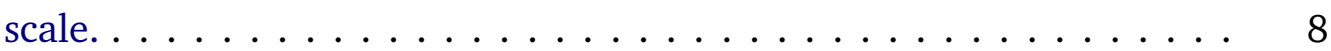

2.2 (a): Accurate rock structure representation using rock Micro-CT.

(b): Simplified porous representations.

(c) Continuum approach to porous media. . . . . . . . . . . . 9

2.4 Darcy's experiment . . . . . . . . . . . . . . . . . . . . . . . . 12

2.5 Schematic streamlines in porous media . . . . . . . . . . . . . 13

2.6 (a): Typical relative permeability curves for the oil-water system.

(b): Hysteresis on relative permeability curves. . . . . . . . . . . . 18

2.7 Comparative between Brook-Corey and Van Genuchten relative permeability correlations. . . . . . . . . . . . . . . . 20

2.8 Hysteresis in capillary pressure curves. . . . . . . . . . . . . . . . . 21

2.9 Comparative between Brooks-Corey and Van Genuchten models for capillary pressure correlation. . . . . . . . . . . . . . . 22

3.1 Five-point finite differences stencil on a staggered grid. . . . . . . . . . 26

3.2 TVD limiter functions. . . . . . . . . . . . . . . . . . . . . 28

3.3 Impact of the flux limiter in the solution of the Buckley-Leverett problem. 29

3.4 Solutions used for scheme order estimation using Richardson extrapolation. . . . . . . . . . . . . . . . . . . . 30

3.5 Stencil used in face and corner boundaries. . . . . . . . . . . . . . 31

3.6 Impact of mesh refinement in the solution. . . . . . . . . . . . . . . 32

3.7 Evolution of the GMRES tolerance, pressure error and total divergence error with the number of GMRES iterations. . . . . . . . . . . . . . . 39

3.8 Evolution of GMRES and divergente tolerance error. . . . . . . . . . 40

4.1 Case 1. Water saturation profiles for the Buckley-Leverett solution. . . 43

4.2 Case 2. Absolute permeability field and gas saturation distributions. . . 45

4.3 Case 2. Error in saturation distribution with gravity. . . . . . . . . . . . 45 
4.4 Case 2. Error in saturation distribution with no gravity. . . . . . . . . . . 46

4.5 Case 2. Gas production history. . . . . . . . . . . . . . . . . . 46

4.6 Case 2. Iterative solver efficiency. . . . . . . . . . . . . . . . . 47

4.7 Case 3. Absolute permeability and wetting phase saturation distribution. 48

4.8 Case 3. Error distribution for different tolerance values. . . . . . . . . . 49

4.9 Case 3. Iterative solver efficiency. . . . . . . . . . . . . . . . . . 50

4.10 Case 4a. Permeability and wetting phase distribution. . . . . . . . . 51

4.11 Case 4a. Error distribution for different values of solver tolerance. . . . 51

4.12 Case 4b. Permeability and wetting phase distribution. . . . . . . . . . 52

4.13 Case 4b. Error distribution for different values of solver tolerance. . . . 52

4.14 Case 4a. Permeability and wetting phase distribution. . . . . . . . . 53

4.15 Case 4c. Error distribution for different values of solver tolerance. . . . 54

4.16 Case 4. Efficiency of iterative solver for different tolerances. . . . . . . . 54

4.17 Case 5. Absolute permeability and water distribution. . . . . . . . . 56

4.18 Case 5. Finger structure for different solver tolerances. . . . . . . . . 57

4.19 Case 5. Impact of solver tolerance. . . . . . . . . . . . . . . . 58

4.20 Case 6. Permeability distribution for the SPE 10 dataset 2 . . . . . . 59

4.21 Case 6. Porosity distribution for the SPE 10 dataset 2 . . . . . . . . . 60

4.22 Case 6.Pressure distribution and water distribution. . . . . . . . . . 60

4.23 Case 6. Water cut history. . . . . . . . . . . . . . . . . . 61

4.24 Convergence history for the GMRES linear solver. . . . . . . . . . . . . 62

4.25 Impact of preconditioner on the GMRES iterative solver. . . . . . . . . . 62

4.26 Impact of GMRES restart iteration with ILU(0) preconditioner. . . . . . 63

4.27 Impact of GMRES restart iteration with ILUT preconditioner. . . . . . . 63

5.1 Rock file visualized with Paraview. . . . . . . . . . . . . . . . . . 68

5.2 Darcy2D code simplified diagram. . . . . . . . . . . . . . . . . . 69

5.3 2D solver multithread scalability. . . . . . . . . . . . . . 73

A.1 AOS and SOA scalability with low arithmetic load loop . . . . . . . . 83

A.2 AOS and SOA loop memory access profile. . . . . . . . . . . . . 84

A.3 AOS and SOA scalability with high arithmetic load loop . . . . . . . 85

B.1 Example of Fortran-Python plot $\ldots \ldots \ldots \ldots$ 


\section{chapter 1}

\section{INTRODUCTION}

\subsection{The problem of reservoir simulation}

A common misconception in oil and gas recovery processes is that the hydrocarbons are found in a kind of underground lakes and have to be pumped out of the reservoir in a similar way water is pumped out of a storage tank. This is absolutely not the case. Hydrocarbons are found impregnating the void space of porous or fractured rocks, such as sandstone, carbonates or dolomite. The pores of this kind of rocks are connected forming a highly irregular microscopic net. The volume of pores is typically small (1-20 \%) [Bear, 1972]. The oil or gas impregnating the rock can flow through these intricate paths under high pressure differentials. The ability of the rocks to permit the flow of fluids through the pore net varies hugely between different rock types, or even between zones of the same rock due to inhomogeneities or fractures. The typical size of the reservoirs, of the order of magnitude of kilometres, and the influence of rock inhomogeneities make a hard task to accurately characterize and model the porous media [Aarnes et al., 2007]. In addition to the complicated nature of the porous media, the oil or gas are a complex mixture of hydrocarbons, hard to characterize, with chemical and physical properties that vary widely between reservoirs. Initially, the crude oil is at reservoir conditions, at a temperature given by the geothermal gradient $\left(\sim 30^{\circ} \mathrm{C} / \mathrm{km}\right)$ and a typical pressure of hundreds of bar. Pressure and temperature of the crude oil vary from reservoir to storage conditions during the extraction and the physical properties of the hydrocarbon vary during this phase including, often, phase changes.

The pressures found in reservoirs are extremely high, normally these pressures are high enough to force the fluids to flow through the porous media and out of the wells without much pumping effort. This type of recovery, is named primary recovery. As the crude oil is extracted from the reservoir, the pressure drops and the production 
rate decreases as the gradient driving the flux gets lower. The amount of hydrocarbons extracted from the reservoir within this type of recovery is typically not greater than 30\% [Ewing, 1983]. After this phase, a fluid, typically water, is injected in some wells to increase the production rate in the remaining ones. This injection maintains the reservoir pressure gradients and the injected fluid displaces the crude towards the producers. This phase is known as secondary recovery. Water flooding is a complex phenomena, the interface between oil and water is unstable and fingers of the less viscous phase are formed connecting injectors and producers, reducing the efficiency of oil displacement. Typically, a $50 \%$ of the crude remains in the reservoir after this phase [Ewing, 1983]. Other techniques to increase the recovery, known as Enhanced Oil Recovery (EOR) are used in the tertiary recovery phase. The techniques used in this phase include additives addition to increase the displacement efficiency, gas injection to force miscible displacement, steam injection to reduce oil viscosity or air injection and in-situ combustion for increasing pressure and temperature in the reservoir or even microbial agents injection [Amarnath, 1999]. Physical and chemical processes involved in this tertiary phase are complex and extremely difficult to reproduce mathematically.

\subsection{Motivation}

Numerical reservoir simulators are tools widely used by the oil industry and have become the main instrument for evaluating recovery efficiency and economical viability of new oil-drilling. Simulation of multiphase fluid flow in porous media is currently a topic of interest in many areas, not only in reservoir simulation, but also in other fields like hydrology and groundwater flow or waste management.

In reservoir simulation, far-field areas are well-understood and fairly resolved with current simulation tools but near wellbore regions are normally under-resolved and become a source of inaccuracy in the resolution of the fluid physics in this zone [Ding, 2011; Dumkwu et al., 2012]. The under-resolved physics is modelled by including several parameters, adjusted using history matching, such as the well skin factor or the productivity index [Dumkwu et al., 2012]. However, the logarithmic pressure drop in the surrounding wellbore region, as well as the complex thermodynamic behaviour of oil often leads to sudden variations in flow properties. These changes modify the production rates in a determinant way and can be hardly predicted by parametric wellbore models. Moreover, new techniques in oil drilling, including crosswise and horizontal wellbores and enhanced oil recovery techniques, increase the uncertainties associated to these models [Leeftink et al., 2015] making very difficult to accurately predict the recovery efficiencies in the mid and long terms. In order to reduce these uncertainties, it is therefore crucial to accurately simulate the near wellbore region in 
the most efficient way.

In terms of numerical simulation, the Fully Implicit Method (FIM) [Collins and Nghiem, 1992; Dawson et al., 1997; Lee et al., 2011; Sun et al., 2009; Tan and Kalogerakis, 1991; Wu and Qin, 2009; Yang et al., 2016] and the IMplicit Pressure Explicit Saturation (IMPES) method [Chen et al., 2004; da Silva et al., 2016; Hurtado et al., 2006; Kou and Sun, 2010a,b; Sheldon et al., 1959; Stone and Garder Jr., 1961] are the main strategies to solve the system of partial differential equations arising from the discretisation of the mathematical model of the multiphase flow in porous media.

The FIM is regularly used in the simulation of the large far-field reservoir areas. Being unconditionally stable, it allows for selecting large time steps which are able to solve years of operation of complete reservoirs with dozens of producers and injectors in a few hours of computational time [Künze et al., 2013; Liu et al., 2016]. However, FIM is not well suited for detailed simulation, as required in the near wellbore region, where smaller time steps are needed to capture fast dynamic behaviour and coupling with pipe simulators. In this region and despite its limitations, the IMPES method is normally preferred. IMPES is an operator splitting technique [Kou and Sun, 2010b] based on physics, which solves only the pressure equation implicitly, but updates the saturation explicitly. The pressure equation in this approach is formed by substituting the saturation constraint and Darcy's law into the sum of the mass conservation laws. The computational cost (time and memory) required by the IMPES method is smaller than the FIM at each time step. This advantage is more pronounced for problems with a high number of degrees of freedom and high velocity fluctuations, which induce shorter time steps. The explicit treatment of the saturation equation results in a stability limitation for the time step, especially for highly heterogeneous permeable media. It should be noticed that this drawback is not so important if there is a physical constraint in the maximum time step, as it happens in the near wellbore region.

However, the IMPES method is not widely used in industrial problems due to the high computational cost of solving the implicit pressure equation, which roughly represents the 90 per cent of the solver cost [Hurtado et al., 2006]. Therefore, as already mentioned, modelling that region is the preferred option. To circumvent this problem, Chen, Huan an Li [Chen et al., 2004] have proposed an improved IMPES method where the implicit pressure equation is only solved after the variation in the saturation exceeds a certain threshold. The main drawback of this method is a lack of accuracy resulting from updating the saturations with incorrect pressure values which eventually gives rise to nonphysical numerical oscillations of the pressure. This idea has been further extended and improved in the last years [da Silva et al., 2016; Hurtado et al., 2006; Karlo et al., 2009].

In this work, a novel methodology is proposed to improve the performance of the 
IMPES method by using an iterative linear solver, which drastically reduces the computational cost of the pressure equation solver to times of the same order of magnitude of the saturation solver, eventually giving rise to a total reduction of the computational time in a factor of 10, compared to recent IMPES results in the literature. The developed method is referred here as iterative IMPES, while the IMPES using direct solver for pressure equation will be denoted as conventional IMPES.

In addition to the mathematical and numerical models, efforts have been made to obtain an efficient and modular code that can be the base for further development. For that reason, modern object-oriented Fortran and high performance computing (HPC) libraries have been used in the coding phase.

\subsection{Methodology}

Based on the work of Ewing [1983], there are five major stages in the modelling process for reservoir simulation:

1. A physical model of the flow processes is developed incorporating as much physics as is deemed necessary to describe the essential phenomena. On the one hand, a too detailed model will produce an excessively complex mathematical model and the need of data to feed the model that probably will not be available. On the other hand, a too simple model can neglect important effects and produce completely wrong results.

2. A mathematical formulation of the physical model is obtained, usually involving coupled systems of nonlinear partial differential equations.

3. Once the properties of the mathematical model, such as existence, uniqueness, and regularity of the solution, are sufficiently well understood and the properties seem compatible with the physical model, a discretised numerical model of the mathematical equations is produced. A numerical model is determined which has the required properties of accuracy and stability and which produces solutions representing the basic physical features as well as possible without introducing spurious phenomena associated with the specific numerical scheme.

4. A computer program capable of efficiently performing the necessary computations for the numerical model is sought.

5. The output of the computer program which gathers the results of the whole model needs to be validated against experimental data, analytical models or other available and validated results. 
The following chapters expand on the methodology summarised in this section. In Chapter 2 the first two points are expounded, the appropriate physical models and simplifications are chosen to describe the multiphase flow through porous media, these models have been expressed in mathematical form for the case of two immiscible fluid phases. In Chapter 3, the discretisation of partial differential equations is detailed and a new method to efficiently solve the discretised system will be analysed for the $2 \mathrm{D}$ case in a regular grid. In Chapter 4, the output of the computer program is validated against analytical solutions and published results. In Chapter 5, the implementation is detailed and computational efficiency is measured and compared with other published methods. 



\section{chapter 2}

\section{MATHEMATICAL MODELS}

\subsection{The porous media}

Porous materials are present everywhere in daily life, however a general definition of porous media is not easy to find. Depending on the application some properties are required to treat a system as porous media. In reservoir and hydrology science one can assume the definition of Bear [1972]:

"A portion of space occupied by heterogeneous or multiphase matter. At least one of the phases comprising this matter is not solid. They may be gaseous and/or liquid phases. The solid phase is called the solid matrix. That space within the porous medium domain that is not part of the solid matrix is referred to as void space or pore space.

The solid phase should be distributed throughout the porous media within the domain occupied by a porous medium; solid must be present inside each representative elementary volume. An essential characteristic of a porous media is that the specific surface of the solid matrix is relatively high. In many respects, this characteristic dictates the behaviour of fluids in porous media. Another basic feature of a porous medium is that the various openings comprising the void space are relatively narrow.

At least some of the pores comprising the void space should be connected. The interconnected space is sometimes termed the effective pore space. As far as flow through porous media is concerned, unconnected pores may be considered as part of the solid matrix. Certain portions of the interconnected pore space may, in fact, also be ineffective as far as flow through the medium is concerned. For example, pores may be dead-end-pores (or blind pores), i.e. pores or channels with no flow through them."

The definition of phase is taken from Bear and Bachmat [1990]. A phase is defined as a chemically homogeneous portion of a system under consideration that is separated 
from other such portions by a definite physical boundary (interface, or inter-phase boundary). There can be only one gaseous phase in a system, as all gaseous phases are completely miscible and do not maintain a distinct boundary between them. We may, however, have more than one liquid phase in a system. Such liquid phases are referred to as immiscible fluids. In Figure 2.1 a schematic representation, at microscopic scale, of two immiscible phases in porous media is outlined.

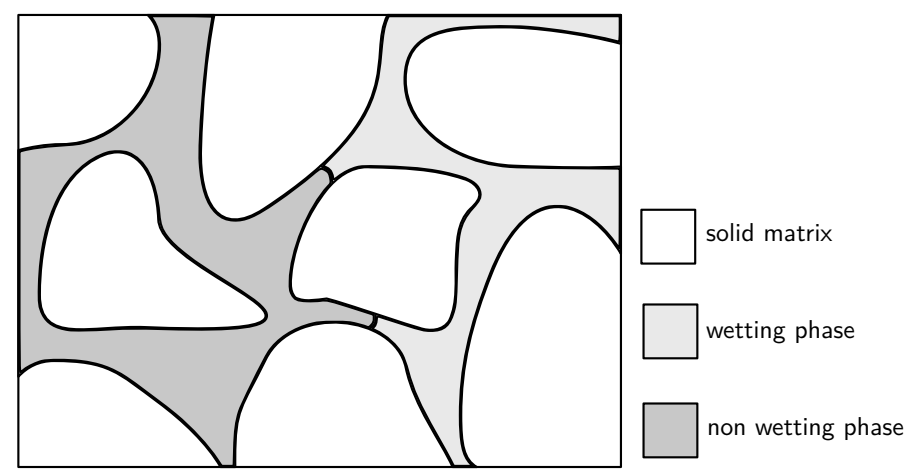

Figure 2.1: Schematic of two immiscible phases on porous media at microscopic scale.

\subsubsection{Continuum approach}

The flow in porous media can be studied at different scales. At a microscopic scale individual grains and channels are visible and fluid phases flood the void space. The huge resolution needed to define accurately the geometry, fluid behaviour and fluid rock interaction at this scale the lead to a extremely costly simulations and restricted to millimetre size domains. Lattice-Boltzmann method is usually chosen for this microscopic-scale simulations [Boek and Venturoli, 2010]. A detailed geometry at microscopic scale is shown in Figure 2.2(a).

There is an intermediate scale, in which porous media is simplified using simpler geometries. This simplified geometries maintain relevant microscopic mean values of properties such as pore diameters, grain size, channels tortuosity and others. Geometry is defined so that desired macroscopic properties are representative of the original geometry. An example of simplified geometries is shown in Figure 2.2(b) [Bowers and Katsube, 2002].

In this work porous media is studied at a macroscopic scale. At this scale we can define average macroscopic properties. It is important the concept of representative elementary volume ( $R E V$ ), this is the smallest volume over which the mean value of the properties (porosity, permeability...) are representative of the whole [Bear, 1972; Muccino et al., 1998]. If a REV can not be determined, the theory related to continuum 


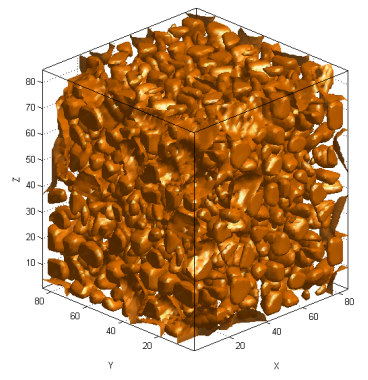

(a)

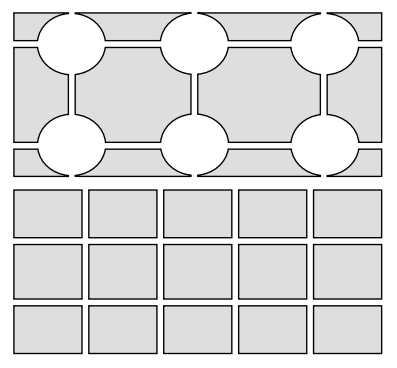

(b)

\begin{tabular}{|l|l|l|}
\hline$\phi_{1}$ & $\phi_{2}$ & $\phi_{3}$ \\
$K_{1}$ & $K_{2}$ & $K_{3}$ \\
\hline$\phi_{4}$ & $\phi_{5}$ & $\phi_{6}$ \\
$K_{4}$ & $K_{5}$ & $K_{6}$ \\
\hline$\phi_{7}$ & $\phi_{8}$ & $\phi_{9}$ \\
$K_{7}$ & $K_{8}$ & $K_{9}$ \\
\hline
\end{tabular}

(c)

Figure 2.2: (a): Accurate rock structure representation using rock Micro-CT. (b): Simplified porous media representations. (c) Continuum approach to porous media

approach of porous media can not be applied. The definition of REV based on porosity can be seen in Figure 2.3. In this work the continuum approach, Figure 2.2(c), is used so it is assumed that a REV exits and every property defined is assumed to be macroscopic.

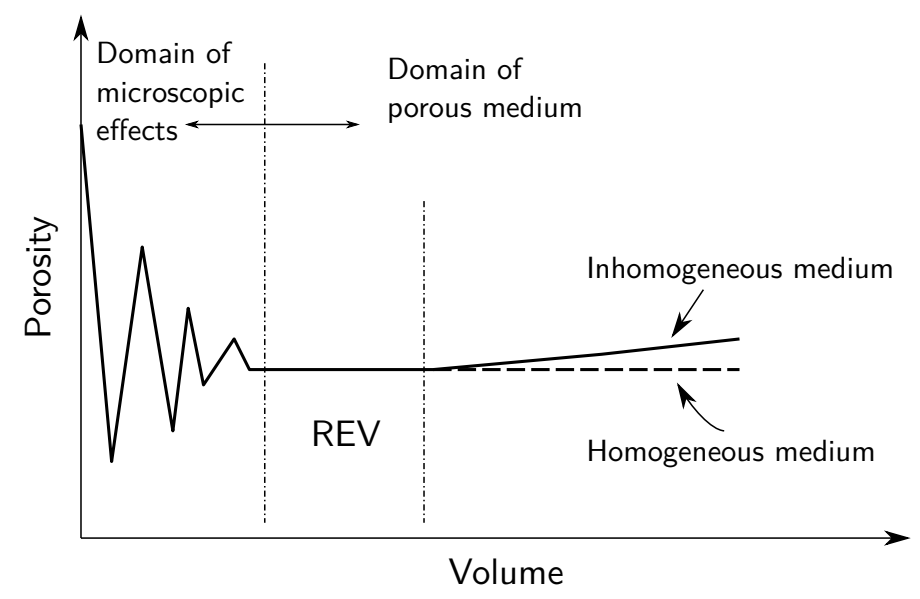

Figure 2.3: Averaged porosity function on averaging volume.

\subsubsection{Properties of porous media}

In this section some relevant properties of porous media are briefly described.

\section{Homogeneity}

An homogeneous material is one that has the same macroscopic properties in every point of the space. On the contrary, an inhomogeneous material will present spatial 
variation of its properties with position.

\section{Anisotropy}

Anisotropy means that some properties of the material will vary with the direction. Properties that can vary with direction at the same point are known as tensor magnitudes.

\section{Porosity}

Porosity is one of the most relevant properties of the porous media, representing the void space that can be filled with the fluid or fluids. Quantitatively, porosity, denoted by the Greek letter $\phi$, is the ratio of the pore volume to the total one:

$$
\phi=\frac{\text { pore volume }}{\text { total volume }} \text {. }
$$

In some porous media, a percentage of porous can be disconnected from the rest, in this work these porous are considered as solid matrix since fluid can not flow through those. Porosity that takes into account only connected porous is also known as effective porosity. Common porosity values of relevant sedimentary materials are listed in Table 2.1 .

Table 2.1: Typical porosity values from [Bear, 1972]

\begin{tabular}{lc}
\hline Sedimentary material & Porosity(\%) \\
\hline Peat soil & $60-80$ \\
Clay & $45-55$ \\
Uniform sand & $30-40$ \\
Gravel & $30-35$ \\
Sandstone & $10-20$ \\
Shale & $1-10$ \\
Limestone & $1-10$ \\
\hline
\end{tabular}

Porosity depends on the pressure if the rock is compressible. Rock compressibility $c_{r}$ is defined as:

$$
c_{r}=\frac{1}{\phi} \frac{\mathrm{d} \phi}{\mathrm{d} p}
$$

where $p$ is the reservoir pressure. Rock compressibility is normally neglected and porosity is assumed to vary only spatially. 


\section{Permeability}

Permeability is the property of the porous media that evaluates the capacity of the media to transmit fluids under a certain pressure gradient. The rock permeability is a tensor property and is denoted by $\boldsymbol{K}$. This property can be estimated using simplified models, Figure 2.2(b), or determined experimentally. The units of permeability are darcy (D) or millidarcy (mD). They are not SI units but are widely used in petrophysics and hydrology. Permeability has dimensional units of $[L]^{2}$. In SI units, 1 darcy $=9.869233 \cdot 10^{-13} \mathrm{~m}^{2}$. Permeability may vary rapidly several orders of magnitude in typical rock formations. Values of permeability in common rock formations are listed in Table 2.2.

Table 2.2: Typical permeability values from [Bear, 1972].

\begin{tabular}{lc}
\hline Soil / Rock & Permeability(mD) \\
\hline Clean sand & $10^{5}-10^{3}$ \\
Peat & $10^{4}-10^{2}$ \\
Very fine sand & $10^{2}-1$ \\
Stratified clay & $10^{2}-0.1$ \\
Sandstone & $10-0.1$ \\
Limestone & $10^{-1}-10^{-3}$ \\
Granite & $10^{-3}-10^{-5}$ \\
\hline
\end{tabular}

Usually, cross terms in $\boldsymbol{K}$ are neglected and the permeability tensor is assumed to be diagonal. This assumption is taken in this work so the permeability tensor has the form:

$$
\boldsymbol{K}=\left[\begin{array}{ccc}
k_{x} & 0 & 0 \\
0 & k_{y} & 0 \\
0 & 0 & k_{z}
\end{array}\right]
$$

\subsection{Flow in porous media}

\subsubsection{Darcy's law}

In 1856 Henry Darcy investigated the flow of water in sand filters in the city of Dijon, France [Simmons, 2008]. A scheme of the experimental set-up is shown in Figure 2.4.

He concluded that the flow rate of water $(Q)$ through the filters was proportional to the height difference $\left(h_{1}-h_{2}\right)$, proportional to the cross sectional area $(A)$ and 
inversely proportional to the filter length $(L)$. The conclusions of the experiment led to the Darcy's formula:

$$
Q=\frac{K A\left(h_{1}-h_{2}\right)}{L}
$$

were $K$ is a proportionality constant.

The $h$ terms can be interpreted as the water piezometric head, those terms describe the sum of pressure and potential energy of water. As detailed in [Bear, 1972], Darcy's formula can be generalised for three dimensional flows in homogeneous isotropic mediums. Darcy's equation under this assumption reads:

$$
\boldsymbol{u}=-\frac{k}{\mu}(\nabla p-\rho \mathbf{g})
$$

where $\boldsymbol{u}$ is the flow per unit area $(Q / A)$ (also known as specific discharge rate or Darcy velocity), $\mu$ is the fluid viscosity, $\nabla p$ is the pressure gradient, $\rho$ is the fluid density, $\boldsymbol{g}$ is the vector of gravity acceleration and $k$ is a positive coefficient of proportionality known as media permeability.

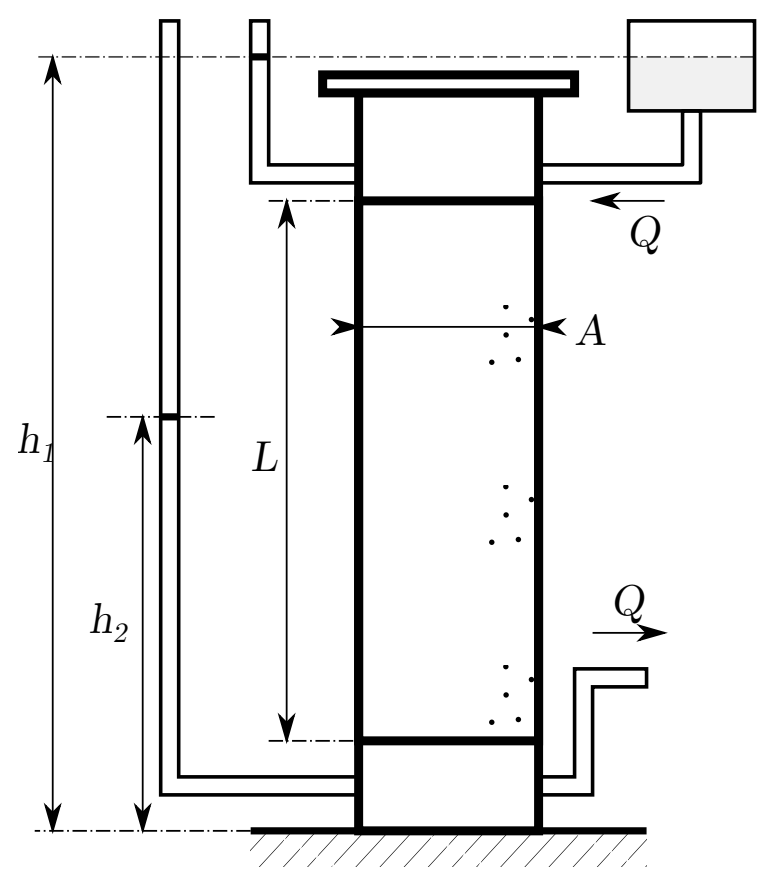

Figure 2.4: Darcy's experiment [Bear, 1972].

Darcy's law can also be derived from Navier-Stokes momentum equation under some assumptions as detailed in the work of Whitaker [1986]. 


\subsubsection{Validity of Darcy's law}

Linear relationship between hydraulic gradient and specific discharge rate fails at high rates. Therefore the range of validity of Darcy law has to be established. Bear [1972] determines the region of validity using the Reynolds number for the flow trough the porous media defined as:

$$
R e=\frac{u d}{v},
$$

where $v$ is the kinematic viscosity of the fluid $(v=\mu / \rho), u$ the specific discharge rate and $d$ some characteristic dimension of the rock grains (often grain mean diameter). In practically all cases, Darcy's law is valid as long as the Reynolds number based on average grain diameter does not exceed some value between 1 and 10 i.e., when the fluid flows at Stokes regime (Figure 2.5(a)). For higher Reynolds numbers, the deviation from Darcy's law is caused by inertial forces that are negligible at low Reynolds numbers. Inertial forces are proportional to the square of the velocity and independent of the viscosity and induce secondary structures and detached flow regions (Figure 2.5(b)). Between $R e=1$ and $R e=10$ the validity of Darcy's law is determined by the behaviour of the flow through the porous media, mainly dependent on the porous structure. The flow in this region, however, remains laminar.

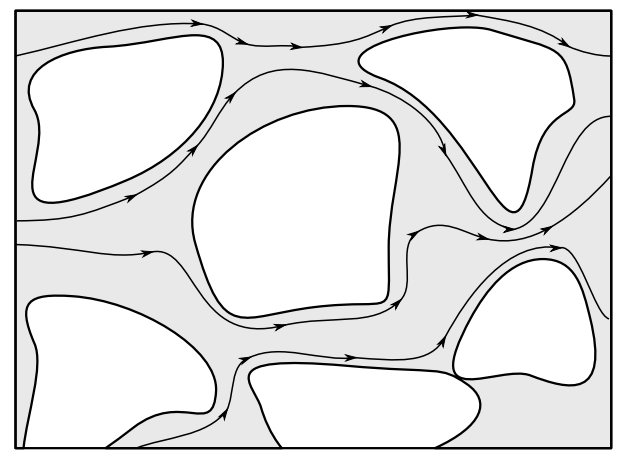

(a)

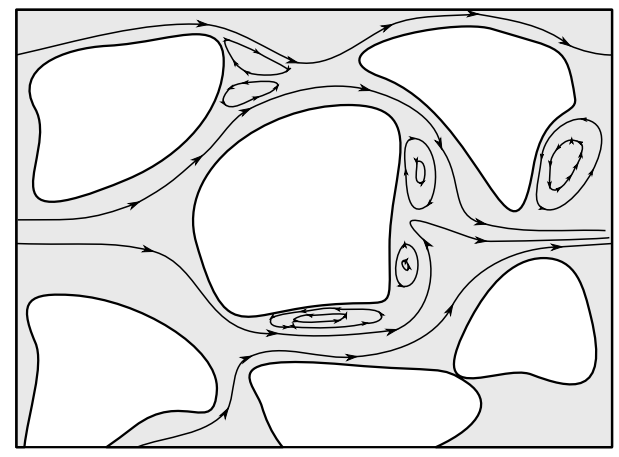

(b)

Figure 2.5: Schematic streamlines of fluid through porous media. (a): Stokes flow at $R e<1$. (b): Detached flow zones at $R e>10$ caused by inertial forces.

At higher Reynods numbers ( $R e>60 \sim 150$ ) flow inside the porous media becomes turbulent and the linear relation between pressure gradient and flow rate is no longer applicable.

Darcy's law presents a lower limit too. There exist a minimum gradient below which the flow is negligible. This pressure threshold is attributed by some authors to non-Newtonian liquid viscosity, to the interaction of the fluid with the solid surface or crosscurrents caused by the streaming potential. In gases, when the mean free path of 
the molecules approaches the dimension of the porous channel, a movement known as Knudsen flow may appear [Webb, 2006].

In reservoir simulation the flow will be in the region of validity of Darcy's law with exception of zones of high speed such as perforations or fractures where Darcy's law may be not applicable. In these regions, non linear velocity correlations, such as the Forchheimer equation,

$$
-\frac{\mathrm{d} p}{\mathrm{~d} x}=\frac{\mu u}{k}+\beta \rho u^{2},
$$

where $u$ is the velocity and $\beta$ is the non-Darcy coefficient, may be required [Zeng and Grigg, 2006].

In this work, the Darcy equation with no corrections is used.

\subsubsection{Single-phase flow}

The flow in porous media at macro scale is governed by volume averaged equations. The basic principle describing one phase flow is the mass conservation (or continuity), which expressed in differential form reads:

$$
\frac{\partial(\phi \rho)}{\partial t}+\nabla \cdot(\rho \mathbf{u})=\hat{q}
$$

In (2.8), $\phi$ is the porosity (effective porosity) as defined in (2.1). The density of the fluid $\rho$ is assumed to be constant in this work, i.e., incompressible flow. $\boldsymbol{u}$ is the macroscopic velocity (Darcy velocity). At microscopic level velocity takes a mean value of $\boldsymbol{u}_{m}=\mathbf{u} / \phi . \hat{q}$ represents the mass source terms.

Inserting Darcy's equation, (2.5), into mass conservation equation, (2.8), yields the equation for the pressure,

$$
\frac{\partial(\phi \rho)}{\partial t}+\nabla \cdot\left[\rho \frac{K}{\mu}(\nabla p-\rho \mathbf{g})\right]=\hat{q}
$$

Assuming incompressibility both for the fluid and for the rock the temporal derivative vanishes and the equation (2.9) reduces to:

$$
\nabla \cdot\left[\frac{\boldsymbol{K}}{\mu}(\nabla p-\rho \mathbf{g})\right]=q,
$$

where the source terms $q$ are now volumetric.

The elliptic partial differential equation (2.10) needs to be completed with boundary conditions for the pressure. The pressure field can be computed by solving equation (2.10) then, using (2.5), the velocity field can be recovered. 


\subsection{Multi-phase flow in porous media}

Similar to single-phase flow the fundamental model behind flow equations is the mass conservation. In this case, the mass conservation for a generic number of phases can be expressed as:

$$
\frac{\partial}{\partial t}\left(\phi \rho_{i} s_{i}\right)+\nabla \cdot\left(\rho_{i} \mathbf{u}_{i}\right)=q_{i}, \quad i=\text { phase } 1 \ldots \text { phase } \mathrm{n}
$$

In equation (2.11), $s_{i}$ represents the saturation of phase $i$ that is the portion of void space occupied by the phase $i$ in a control volume. From the definition of porous media, all the void space is filled with fluid phase, which in mathematical form is expressed as:

$$
\sum_{i} s_{i}=1, \quad i=\text { phase } 1 \ldots \text { phase } \mathrm{n} .
$$

In multi-phase flow, velocities can be modelled, as well as in single-phase, using Darcy's law [Bear, 1972; Coats et al., 1969], which now relates the velocity of each phase $\mathbf{u}_{i}$ with the phase pressure field $p_{i}$,

$$
\boldsymbol{u}_{i}=-\frac{\boldsymbol{K} k_{r i}}{\mu_{i}}\left(\nabla p_{i}-\rho_{i} \boldsymbol{g}\right)
$$

In (2.13), $k_{r i}$ is the relative permeability, a factor that takes into account the presence of other phases in the phase permeability and is discussed in section 2.3.2.

\subsubsection{Immiscible two-phase flow}

There are multitude of problems of interest in reservoir simulation that can be reduced to a two-phase immiscible flow. Gas phase and liquid hydrocarbon phase with immobile water (connate water saturation) can be treated as a pure two-phase flow and is important in primary oil recovery. Hydrocarbon liquid phase (black oil) and water is a typical scenario in secondary recovery simulations.

In this work, where two phases are present, each phase is defined as wetting or non-wetting phase. In the case of water and oil, water is the wetting and oil the nonwetting phase. In the case of oil and gas, oil is the wetting and gas the non wetting phase. 
For two phases, the system (2.11) has the following form:

$$
\begin{gathered}
\frac{\partial\left(\phi \rho_{w} s_{w}\right)}{\partial t}+\nabla \cdot\left(\rho_{w} \boldsymbol{u}_{w}\right)=q_{w} \\
\frac{\partial\left(\phi \rho_{n} s_{n}\right)}{\partial t}+\nabla \cdot\left(\rho_{n} \boldsymbol{u}_{n}\right)=q_{n}
\end{gathered}
$$

where the subscripts $w$ and $n$ refer to the wetting and non-wetting phase respectively. Conservation equations (2.14) can be expanded in order to ease further simplifications:

$$
\begin{gathered}
\frac{\partial \phi}{\partial t} s_{w}+\phi \frac{\partial s_{w}}{\partial t}+\phi \frac{s_{w}}{\rho_{w}} \frac{\partial \rho_{w}}{\partial t}+\nabla \cdot \boldsymbol{u}_{w}+\frac{\boldsymbol{u}_{w} \cdot \nabla \rho_{w}}{\rho_{w}}=\frac{q_{w}}{\rho_{w}} \\
\frac{\partial \phi}{\partial t} s_{n}+\phi \frac{\partial s_{n}}{\partial t}+\phi \frac{s_{n}}{\rho_{n}} \frac{\partial \rho_{n}}{\partial t}+\nabla \cdot \boldsymbol{u}_{n}+\frac{\boldsymbol{u}_{n} \cdot \nabla \rho_{n}}{\rho_{n}}=\frac{q_{n}}{\rho_{n}} .
\end{gathered}
$$

A pressure equation similar to the one in single-phase flow (2.10) can be deduced by adding continuity equations and using (2.12). The resulting equation reads:

$$
\nabla \cdot\left(\boldsymbol{u}_{w}+\boldsymbol{u}_{n}\right)+\frac{\partial \phi}{\partial t}+\phi \frac{s_{w}}{\rho_{w}} \frac{\partial \rho_{w}}{\partial t}+\phi \frac{s_{n}}{\rho_{n}} \frac{\partial \rho_{n}}{\partial t}+\frac{\boldsymbol{u}_{w} \cdot \nabla \rho_{w}}{\rho_{w}}+\frac{\boldsymbol{u}_{n} \cdot \nabla \rho_{n}}{\rho_{n}}=q .
$$

Multiphase expression of Darcy's law (2.13) can be introduced in (2.16) in order to get one equation for the pressures. To simplify the resulting expression some variables are introduced. The mobility of the phase $i$ is defined as,

$$
\lambda_{i}=\frac{k_{r i}}{\mu_{i}}
$$

and the phase compressibility is defined as:

$$
c_{i}=\frac{1}{\rho_{i}} \frac{\mathrm{d} \rho_{i}}{\mathrm{~d} p_{i}}
$$

Using equations (2.13), (2.17) and (2.18), all the derivatives in (2.16) can be written in terms of derivatives of the pressure: 


$$
\begin{aligned}
\nabla \cdot\left(\boldsymbol{u}_{w}+\boldsymbol{u}_{n}\right) & =-\nabla \cdot\left[\boldsymbol{K} \lambda_{w}\left(\nabla p_{w}-\rho_{w} \boldsymbol{g}\right)+\boldsymbol{K} \lambda_{n}\left(\nabla p_{n}-\rho_{n} \boldsymbol{g}\right)\right] \\
\frac{\partial \phi}{\partial t} & =\frac{\phi}{\phi} \frac{\partial \phi}{\partial p} \frac{\partial p}{\partial t}=c_{r} \phi \frac{\partial p}{\partial t}, \\
\phi \frac{s_{i}}{\rho_{i}} \frac{\partial \rho_{i}}{\partial t} & =\phi s_{i} \frac{1}{\rho_{i}} \frac{\partial \rho_{i}}{\partial p_{i}} \frac{\partial p_{i}}{\partial t}=c_{i} \phi s_{i} \frac{\partial p_{i}}{\partial t}, \\
\frac{\boldsymbol{u}_{i} \cdot \nabla \rho_{i}}{\rho_{i}} & =-\boldsymbol{K} \lambda_{i}\left(\nabla p_{i}-\rho_{i} \boldsymbol{g}\right) \cdot \nabla p_{i} \frac{1}{\rho_{i}} \frac{\mathrm{d} \rho_{i}}{\mathrm{~d} p_{i}}=-c_{i} \boldsymbol{K} \boldsymbol{\lambda}_{i}\left(\nabla p_{i}-\rho_{w} \boldsymbol{g}\right) \cdot \nabla p_{i},
\end{aligned}
$$

with $i=w, n$.

Gathering all the terms, the pressure equation for two phases reads:

$$
\begin{gathered}
-\nabla \cdot\left[\boldsymbol{K} \lambda_{w}\left(\nabla p_{w}-\rho_{w} \boldsymbol{g}\right)+\boldsymbol{K} \lambda_{n}\left(\nabla p_{n}-\rho_{n} \boldsymbol{g}\right)\right]+ \\
c_{r} \phi \frac{\partial p}{\partial t}+c_{w}\left[\phi s_{w} \frac{\partial p_{w}}{\partial t}-\boldsymbol{K} \lambda_{w}\left(\nabla p_{w}-\rho_{w} \boldsymbol{g}\right) \cdot \nabla p_{w}\right]+ \\
c_{n}\left[\phi s_{n} \frac{\partial p_{n}}{\partial t}-\boldsymbol{K} \lambda_{n}\left(\nabla p_{n}-\rho_{n} \boldsymbol{g}\right) \cdot \nabla p_{n}\right]=q .
\end{gathered}
$$

\subsubsection{Relative permeability}

When two or more fluid phases are flowing through a porous media, the void space is occupied for both phases simultaneously and the effective permeability for each phase is reduced. The concept of relative permeability of a phase $i$, or $k_{r i}$, quantifies this permeability reduction. Mathematically this parameter is defined as:

$$
k_{i}=k k_{r i},
$$

where $k_{i}$ is the effective permeability for each phase $i$ regarding to Darcy's law and $k$ is the permeability of the media or absolute permeability.

Relative permeabilities depend on the nature of the fluids (wettability, viscosity) and the porous media. A typical set of relative permeability curves for a given material and fluids is shown in Figure 2.6.

Derivation of these curves is difficult and normally they have to be obtained experimentally for each fluid pair and material.

Another important effect of the coexistence of two phases is the existence of a minimal saturation for each phase below that the phase remains immobile. This minimal saturation is know as residual saturation: $S_{w, r}$ for the wetting phase and $S_{n, r}$ for the non-wetting one. These points can be observed in figure 2.6(a) where typical relative permeability curves are plotted. In the oil-water system, the irreducible water saturation is also known as connate water saturation. 
Relative permeabilities are nonlinear functions of the saturation and the sum of the relative permeabilities at certain saturation is normally smaller than one $\left(k_{w}+k_{n}<k\right)$.

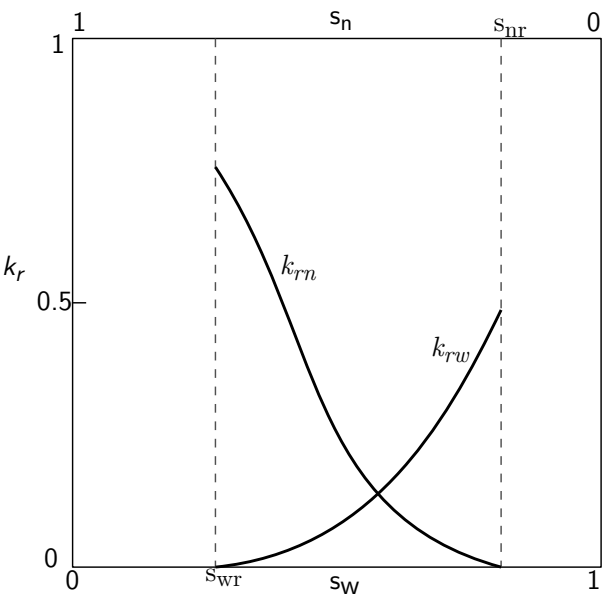

(a)

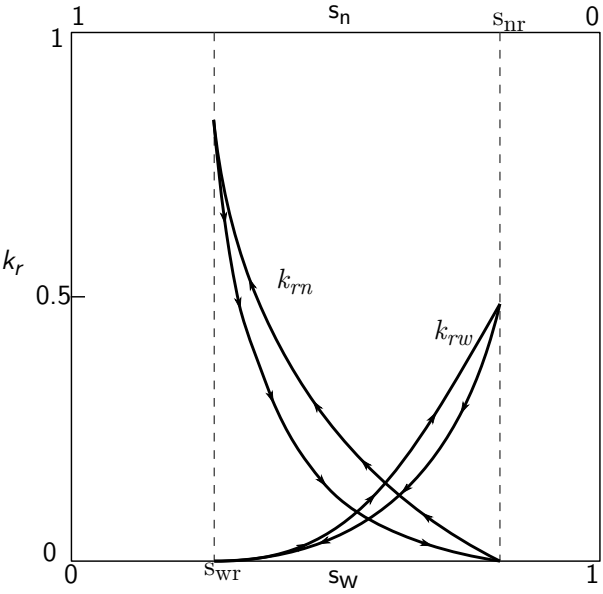

(b)

Figure 2.6: (a): Typical relative permeability curves for the oil-water system. (b): Effect of hysteresis on relative permeability.

Other noticeable behaviour in relative permeability curves is the hysteresis phenomenon. Relative permeability curves are slightly different when the wetting phase is displacing the non-wetting fluid (imbibition) than when it is the non-wetting phase which displaces the wetting fluid (drainage), this effect can be shown in Figure 2.6(b). This phenomenon is highly dependent on the nature of the porous media and is normally neglected in semi-empirical models.

In order to ease the resolution of the equations, several analytical models have been developed. These models correlate the saturation with relative permeability and depend on parameters which have to be determined fitting experimental data or detailed simulations. The models used in the following chapters are presented next.

\section{Brooks and Corey model}

Based on experimental data of several rock formations Brooks and Corey [1964] presented a model based on the idea of effective saturation of the wetting phase, which is defined as:

$$
S_{w, e f f}=S^{*}=\frac{S_{w}-S_{w, r}}{1-S_{n, r}-S_{w, r}} .
$$


The expressions for the relative permeabilities, valid for the system oil-gas, are:

$$
\begin{aligned}
& k_{r n}\left(S^{*}\right)= \begin{cases}k_{r n, \max }\left(1-S_{w, e f f}\right)^{2}\left(1-S_{w, e f f}^{\frac{2+\lambda}{\lambda}}\right) & , 0<S_{w, e f f}<1 \\
0 & , S_{w, e f f} \geq 1 \\
k_{r n, \max } & , S_{w, \text { eff }} \leq 0\end{cases} \\
& k_{r w}\left(S^{*}\right)= \begin{cases}k_{r w, \max } S_{w, \text { eff }}^{\frac{2+3 \lambda}{\lambda}} & , 0<S_{w, \text { eff }}<1 \\
0 & , S_{w, \text { eff }} \leq 0 \\
k_{r w, \max } & , S_{w, \text { eff }} \geq 1\end{cases}
\end{aligned}
$$

The parameter $\lambda$ is called the pore-size distribution index of the media. Typical values for this parameter, reported in [Brooks and Corey, 1964], are $\lambda=1.8 \sim 3.7$ for sand and $\lambda \approx 4.2$ for consolidated sandstone (curves for this last case are plotted in Figure 2.7). A more general expression valid for a generic wetting non-wetting phase system is often used. This correlation depends on two exponents, $n$ and $m$, in the range 1 to 6 ,

$$
\begin{aligned}
& k_{r n}\left(S^{*}\right)= \begin{cases}k_{r n, \max }\left(1-S_{w, \text { eff }}\right)^{n} & , 0<S_{w, \text { eff }}<1 \\
0 & , S_{w, \text { eff }} \geq 1 \\
k_{r n, \max } & , S_{w, \text { eff }} \leq 0\end{cases} \\
& k_{r w}\left(S^{*}\right)= \begin{cases}k_{r w, \max } S_{w, \text { eff }}^{m} & , 0<S_{w, \text { eff }}<1 \\
0 & , S_{w, \text { eff }} \leq 0 \\
k_{r w, \max } & , S_{w, e f f} \geq 1\end{cases}
\end{aligned}
$$

Correlations (2.23) are often referred as modified Brooks-Corey relations.

\section{Van Genuchten model}

This correlation was presented by Van Genuchten [1980] and it is based on the effective saturation of the wetting phase (2.21) and depends only on one parameter, the Van Genuchten exponent $m$ :

$$
\begin{aligned}
& k_{r n}\left(S^{*}\right)= \begin{cases}k_{r n, \max }\left(1-S_{w, e f f}\right)^{\frac{1}{2}}\left(1-S_{w, e f f}^{\frac{1}{m}}\right)^{2 m} & , 0<S_{w, \text { eff }}<1 \\
0 & , S_{w, \text { eff }} \geq 1 \\
k_{r n, \max } & , S_{w, \text { eff }} \leq 0\end{cases} \\
& k_{r w}\left(S^{*}\right)= \begin{cases}k_{r n, \max } S_{w, \text { eff }}^{\frac{1}{2}}\left(1-\left(1-S_{w, \text { eff }}^{\frac{1}{m}}\right)^{m}\right)^{2} & , 0<S_{w, \text { eff }}<1 \\
0 & , S_{w, \text { eff }} \leq 0 \\
k_{r w, \max } & , S_{w, \text { eff }} \geq 1\end{cases}
\end{aligned}
$$




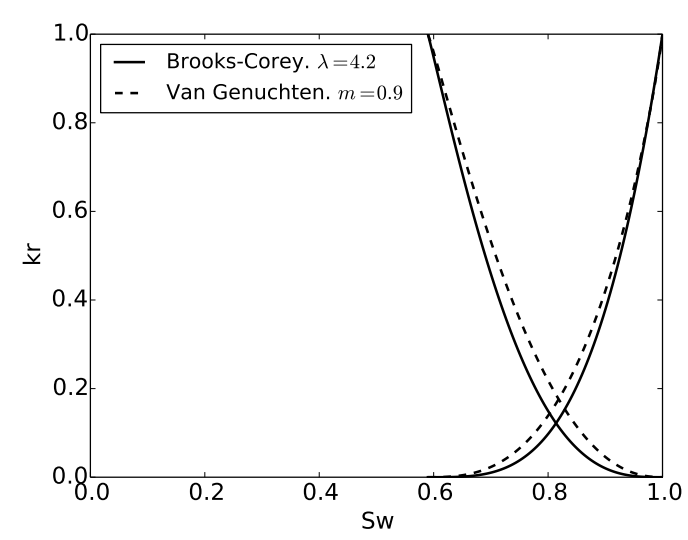

Figure 2.7: Comparative between Brook-Corey and Van Genuchten correlations. Relative permeability curves for hygiene sandstone in the system water-air. Brooks and Corey [1964] and Van Genuchten [1980].

Permeability curves for a typical consolidated sandstone, $m=0.9$, are plotted in figure 2.7.

In Chapter 4, the 2 exponents Brooks and Corey model, (2.23) is used in the validation simulations.

\subsubsection{Capillary pressure}

At microscale, capillary pressure is the pressure jump across an interface between two immiscible fluids,

$$
p_{c}=p_{n}-p_{w} .
$$

The discontinuity magnitude depends on the interface curvature. This dependence is modelled with the Young-Laplace equation,

$$
p_{c}=2 \gamma \frac{1}{r}
$$

where $\gamma$ is the surface tension and $2 / r$ is the radii of curvature.

When considering the continuum approach of the porous media, physical interfaces between phases are not modelled and terms in equations (2.25) and (2.26) have the meaning of statistical average over the void space in a considered region or REV.

In the literature, several empirical and semiempirical expressions are available which attempt to relate the capillary pressure to the media, fluid properties and saturation [Cense and Berg, 2009]. Most of them are based on simplified porous representations (Figure 2.2(b)). For example, for a capillary tube of radius $r$, the capillary 
pressure reads,

$$
p_{c}=\frac{2 \gamma}{r} \cos \theta
$$

In a porous media the value $2 / r$ is replaced by $1 / r^{*}$, where $r^{*}$ is called the hydraulic radius of the capillary [Bear, 1972] and it is a representative value for the media a that point.

In 1941, Leverett [1941] showed that the function,

$$
J\left(S_{w}\right)=\frac{p_{c}\left(S_{w}\right) \sqrt{k / \phi}}{\gamma \cos \theta},
$$

depending on capillary pressure $p_{c}$, surface tension $\gamma$, permeability $k$, porosity $\phi$ and contact angle $\theta$, reduces to a common curve for some different formations. This expression, called J-Leverett function, is the base of the majority of capillary pressure models in porous media.

Similar to relative permeability, capillary pressure shows hysteresis effect, as can be observed in Figure 2.8. The hysteresis is caused mainly due to two effects [Muccino et al., 1998]. Contact angle $\theta$ may have different values if equilibrium is approached by advancing or receding over a surface (raindrop effect) or the presence of many bottlenecks (ink-bottle effect). Hysteresis phenomenon makes the relationship between capillary pressure (or J-Function) and $S_{w}$ not unique.

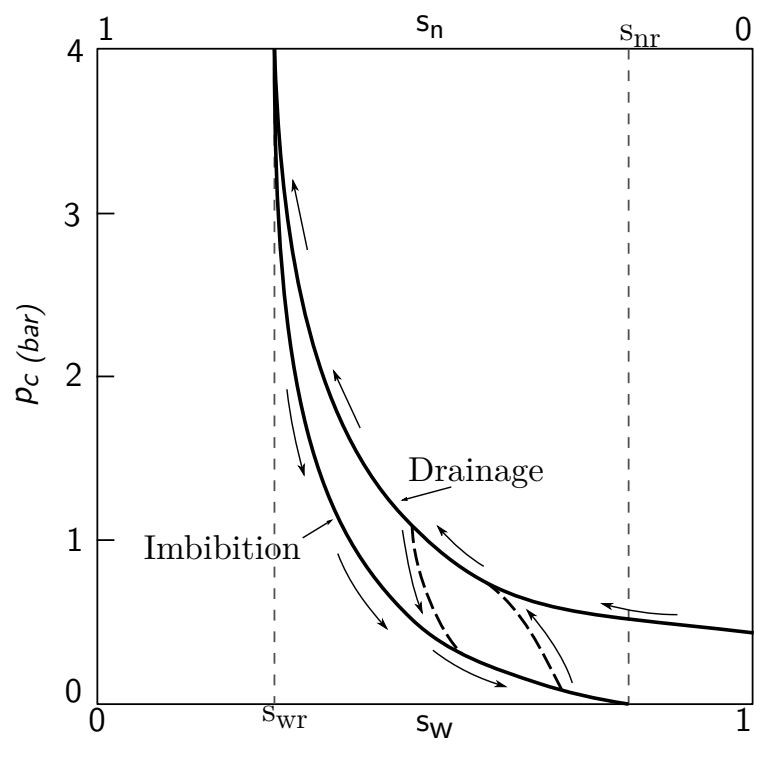

Figure 2.8: Typical hysteresis in capillary pressure curves.

Various authors have proposed analytical expressions for describing the general shape of these curves. Coefficients of these expressions have to be determined by fit- 
ting with experimental data, analytical data from simplified models or detailed multiphase simulations.

The models used in the following chapters of this work are presented below.

\section{Brooks and Corey model}

Presented in the same work [Brooks and Corey, 1964] as the relative permeability model, the capillary pressure depends on the effective saturation of the wetting phase (2.21). The equation reads:

$$
p_{c}\left(S_{e f f}\right)=p_{c, 0} S_{e f f}^{-1 / \lambda}
$$

where $\lambda$ is the pore size distribution index an $p_{c, 0}$ is the entry capillary pressure, corresponding with the capillary pressure at residual non-wetting phase during drainage. A typical capillary pressure curve for a consolidate sandstone, $\lambda=4.2$, is plotted in Figure 2.9.

\section{Van Genuchten model}

This correlation is presented in [Van Genuchten, 1980]. The equation reads:

$$
p_{c}\left(S_{e f f}\right)=p_{c, 0}\left(S_{e f f}^{-1 / m}-1\right)^{1-m}
$$

where $p_{c, 0}$ is the entry pressure and $m$ is the Van Genuchten coefficient used in the relative permeability correlation. The resulting capillary curve for $m=0.9$ (consolidated sandstone) is shown in Figure 2.9.

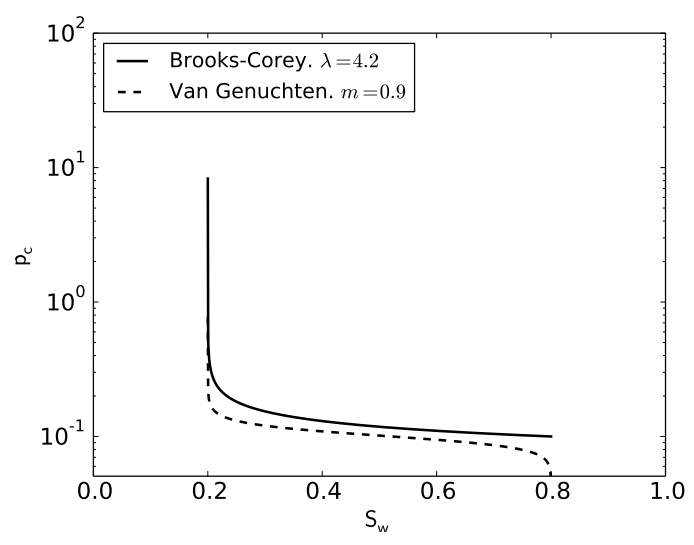

Figure 2.9: Comparative between Brooks-Corey and Van Genuchten models for capillary pressure correlation. 


\section{Logarithmic model with permeability correlation}

This model, proposed by Hoteit and Firoozabadi [2008], is used for validation purpose in $2 \mathrm{D}$ problems. The equation reads:

$$
p_{c}\left(S_{e f f}, k\right)=-\frac{B_{c}}{\sqrt{k}} \log _{10}\left(S_{e f f}\right)
$$

where $k=\sqrt{k_{x} k_{y}}$ is the equivalent permeability and $B_{c}$ is a model parameter. It should be noticed that this model has a dependency with the absolute permeability. This dependency causes discontinuities in the saturation distribution, this effect is shown in the validation cases 3 and 4 (see 4.1.3 and 4.1.4).

\subsubsection{Immiscible incompressible two-phase flow problem}

Equation (2.19) along with mass conservation for each phase (2.8) and permeability and capillary pressure models constitute a closed system of equations. This system, however, can be simplified assuming incompressibility of both phases and rock matrix (i.e., constant porosity). In addition, one of the saturation equations can be substituted by the trivial equation (2.12). Moreover, both phase pressures are correlated through the capillary pressure equation (2.25).

Assuming incompressibility and using the capillary pressure, saturation equations (2.14) and pressure equation (2.19) can be simplified to:

$$
\begin{gathered}
-\nabla \cdot\left[\boldsymbol{K}\left(\lambda_{w}+\lambda_{n}\right) \nabla p_{n}\right]=\nabla \cdot\left[\boldsymbol{K}\left(\lambda_{w} \rho_{w}+\lambda_{n} \rho_{n}\right) \boldsymbol{g}\right]+\nabla \cdot\left(\boldsymbol{K} \lambda_{w} \nabla p_{c}\right)-q, \\
\phi \frac{\partial S_{w}}{\partial t}+\nabla \cdot \boldsymbol{u}_{w}=q_{w} .
\end{gathered}
$$

The system (2.32) - (2.33) is highly coupled. The pressure equation depends on the saturation through mobilities $\lambda$, and saturation depends on pressure through Darcy's law (2.5) for the phase velocity.

The system (2.32) - (2.33), along with the trivial equations,

$$
\begin{aligned}
& S_{n}=1-S_{w}, \\
& p_{w}=p_{n}-p_{c},
\end{aligned}
$$

and proper initial and boundary conditions is closed and has a unique solution [Chen, 2001].

The following chapters address the strategies and methods used to solve the system (2.32) - (2.35) numerically. 



\section{chapter 3}

\section{NUMERICAL METHODS}

In this work, the system of equations (2.32) - (2.35) will be integrated with an implicit explicit (IMEX) method usually known in the literature as IMPES. A second order Total Variation Diminishing (TVD) scheme will be used for the spatial discretisation while a second order Runge Kutta (RK2) method will be used for the temporal integration. For the shake of simplicity and without lack of generality, numerical discretisation is presented in two spatial dimensions.

\subsection{Spatial discretisation}

In this section, the spatial discretisation used in this work will be detailed. A second order finite differences approach on a cartesian staggered grid is used. Scalar properties such as pressure or saturation are evaluated in cell centres, while vectorial magnitudes such as velocities or pressure gradients are evaluated in the midpoints between cell centres. In Figure 3.1, the complete stencil for the $2 \mathrm{D}$ case is shown. In $x$-faces, $\mathrm{x}$-component of vector magnitudes are evaluated while $\mathrm{y}$-components are evaluated at $y$-faces.

Equations in system (2.32) - (2.35) are expressed in divergence form. To numerically solve the system, we define the discretised divergence operator on a mesh point $\boldsymbol{x}_{i, j}=\left(x_{i, j}, y_{i, j}\right)$, at a given time $t^{n}$ as follows:

$$
(\nabla \cdot \boldsymbol{F})_{i, j}^{n}=\frac{F_{i+1 / 2, j}^{n}-F_{i-1 / 2, j}^{n}}{\left(x_{i+1, j}-x_{i-1, j}\right) / 2}+\frac{F_{i, j+1 / 2}^{n}-F_{i, j-1 / 2}^{n}}{\left(y_{i, j+1}-y_{i, j-1}\right) / 2},
$$

where $F^{n}$ are magnitudes evaluated at the faces. For example, $F_{i+1 / 2, j}^{n}$ corresponds to the x-component of the vectorial magnitude $\boldsymbol{F}$ evaluated on the x-face between $\boldsymbol{x}_{i, j}$ and $\boldsymbol{x}_{i+1, j}$. 


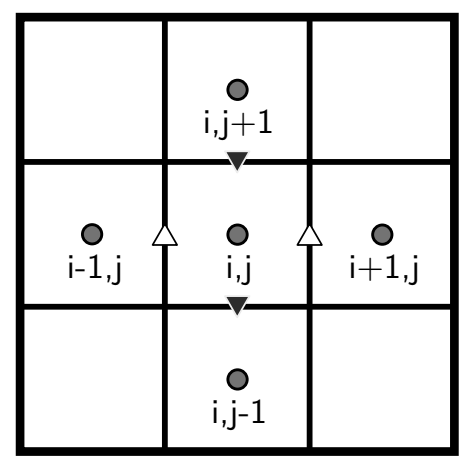

Figure 3.1: 5-point cell centered finite differences stencil. Midpoints or faces are marked with triangles, white for $\mathrm{x}$-faces and black for $\mathrm{y}$-faces.

In order to apply the discretisation in (3.1), involved magnitudes are computed at grid faces. The expressions for the computation of the required magnitudes in the face between $\boldsymbol{x}_{i, j}$ and $\boldsymbol{x}_{i+1, j}$ are detailed below, where $j$ subscripts are dropped for clarity.

Absolute permeability tensor is assumed to be diagonal $\boldsymbol{K}_{i, j}=\left[k_{x}, k_{y}\right]_{i, j} \boldsymbol{l}$. The evaluation of this magnitude in a face between two points, also known as hydraulic conductivity, is computed using the harmonic mean:

$$
k_{x, i+1 / 2}=\frac{2 k_{x, i} k_{x, i+1}}{k_{x, i}+k_{x, i+1}} .
$$

Pressure gradients are computed at the faces using finite differences:

$$
\begin{aligned}
& \left.\nabla p_{n}\right|_{i+1 / 2}=\frac{\left.p_{n}\right|_{i+1}-\left.p_{n}\right|_{i}}{x_{i+1}-x_{i}}, \\
& \left.\nabla p_{c}\right|_{i+1 / 2}=\frac{\left.p_{c}\right|_{i+1}-\left.p_{c}\right|_{i}}{x_{i+1}-x_{i}} .
\end{aligned}
$$

Mobilities, that depend on the advected saturation, are computed using a TVD upwind scheme:

$$
\begin{array}{ll}
\left.\lambda_{\alpha}\right|_{i+1 / 2}=\left.\lambda_{\alpha}\right|_{i}-\varphi\left(\left.\lambda_{\alpha}\right|_{i}-\frac{\left.\lambda_{\alpha}\right|_{i}+\left.\lambda_{\alpha}\right|_{i+1}}{2}\right) & \text {,if } \boldsymbol{u}_{\alpha}>0 \\
\left.\lambda_{\alpha}\right|_{i+1 / 2}=\left.\lambda_{\alpha}\right|_{i+1}-\varphi\left(\left.\lambda_{\alpha}\right|_{i+1}-\frac{\left.\lambda_{\alpha}\right|_{i}+\left.\lambda_{\alpha}\right|_{i+1}}{2}\right) & \text {,if } \boldsymbol{u}_{\alpha}<0 \\
\left.\lambda_{\alpha}\right|_{i+1 / 2}=\frac{\left.\lambda_{\alpha}\right|_{i}+\left.\lambda_{\alpha}\right|_{i+1}}{2} & \text {,if } \boldsymbol{u}_{\alpha}=0
\end{array}
$$

where $\varphi$ is a second order limiter based on the saturation. 
Finally, velocities are computed using the variables previously evaluated at the faces:

$$
\begin{aligned}
& \left.u_{w}\right|_{i+1 / 2}=-\left.\lambda_{w}\right|_{i+1 / 2}\left(\left.\nabla p_{n}\right|_{i+1 / 2}-g_{x} \rho_{w}-\left.\nabla p_{c}\right|_{i+1 / 2}\right) \\
& \left.u_{n}\right|_{i+1 / 2}=-\left.\lambda_{n}\right|_{i+1 / 2}\left(\left.\nabla p_{n}\right|_{i+1 / 2}-g_{x} \rho_{n}\right) .
\end{aligned}
$$

where $g_{x}$ is the x-component of the gravity vector $\boldsymbol{g}$.

This formulation in conservative form, guarantee the conservation of the volume of each phase, this property is crucial on long runs in reservoir simulation.

Introducing discretisation (3.1)-(3.6) into the pressure equation (2.32), we obtain a system of linear equations, with the non-wetting pressure values on each point as unknowns. The system can be expressed in matrix form:

$$
A p_{n}=b
$$

where $A$ is the system matrix, that contains the total mobilities and permeabilities (LHS of equation (2.32)), $\boldsymbol{p}_{\boldsymbol{n}}$ is the vector with the values of non-wetting pressure $\left.p_{n}\right|_{i, j}$ in all the points of the domain, and $\boldsymbol{b}$ contains the terms related to flux sources, gravity and capillary pressure (RHS of equation (2.32)).

\section{Limiters}

Slope limiters are functions that weight first order and a higher order discretisations to avoid oscillations near sharp gradients, reducing the order locally, while maintaining high order in smooth regions [Sweby, 1984]. In this work, limiters are defined as functions of the ratio between consecutive saturation gradients, $r$, evaluated at the faces. $r_{i+1 / 2}$ is defined as:

$$
\begin{aligned}
& r_{i+1 / 2}=\frac{S_{w, i}-S_{w, i-1}}{S_{w, i+1}-S_{w, i}} \quad \text { if } \boldsymbol{u}_{w}>0 \\
& r_{i+1 / 2}=\frac{S_{w, i+2}-S_{w, i+1}}{S_{w, i+1}-S_{w, i}} \quad \text { if } \boldsymbol{u}_{w}<0 \\
& r_{w, i+1 / 2}=1 \quad \text {,if } \boldsymbol{u}_{w}=0
\end{aligned}
$$

There is not a general limiter function that works well for every problem and some popular TVD limiters: MINMOD, Van Leer, Koren and Superbee have been tested. The limiting functions have the following expressions: 


$$
\begin{aligned}
& \text { Minmod: } & \varphi_{m m} & =\max [0, \min (1, r)], \\
& \text { VanLeer: } & \varphi_{v l} & =\frac{r+|r|}{1+r}, \\
& \text { Koren: } & \varphi_{k} & =\max [0, \min (2 r,(2+r) / 3,2)], \\
& \text { Superbee: } & \varphi_{s b} & =\max [0, \min (2 r, 1, \min (r, 2))] .
\end{aligned}
$$

In Figure 3.2, the shape of the limiter function is shown. Minmod limiter is the most diffusive of the second order TVD limiters while Superbee is the least diffusive one.

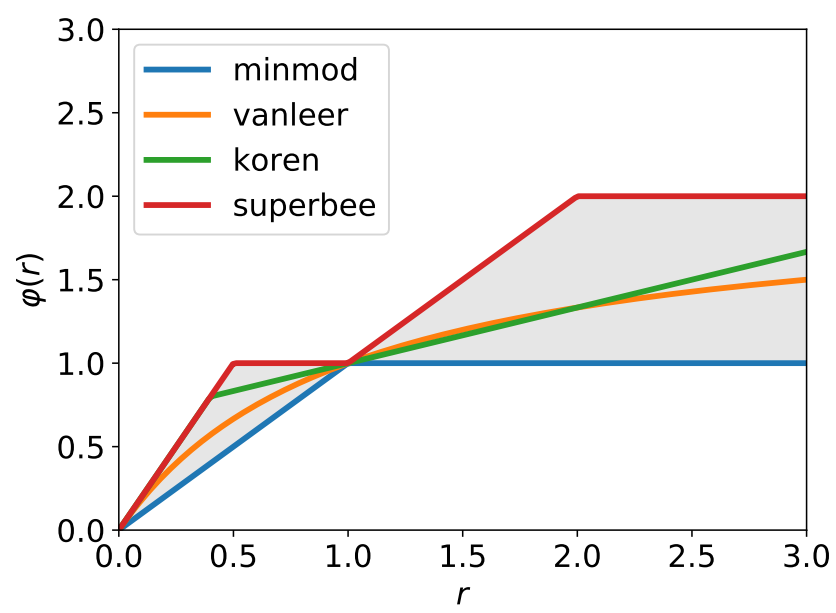

Figure 3.2: Limiter functions. Shaded region corresponds to second order TVD limiters.

In Figure 3.3 the impact of the limiter for the Buckley Leverett problem [Buckley and Leverett, 1942] is shown. All the limiters capture accurately sharp discontinuities, as shown in Figure 3.3-left. It can be observed that less diffusive limiters (Superbee and Koren) tend to square the jump as reported by Kuzmin [2006] and others. In soft jumps, as can be observed in Figure 3.3-right, less diffusive limiters reproduce more accurately the analytical solution.

Among the tested slope limiters, Van Leer has shown the best behaviour in sharp fronts. Koren and Superbee have shown convergence problems in complex scenarios with capillary effects and sharp permeability variations. Thus Van leer limiter has been used in all the validation and analysis cases.

A detailed review of TVD limiters can be found in the work of Sweby [1984]. 

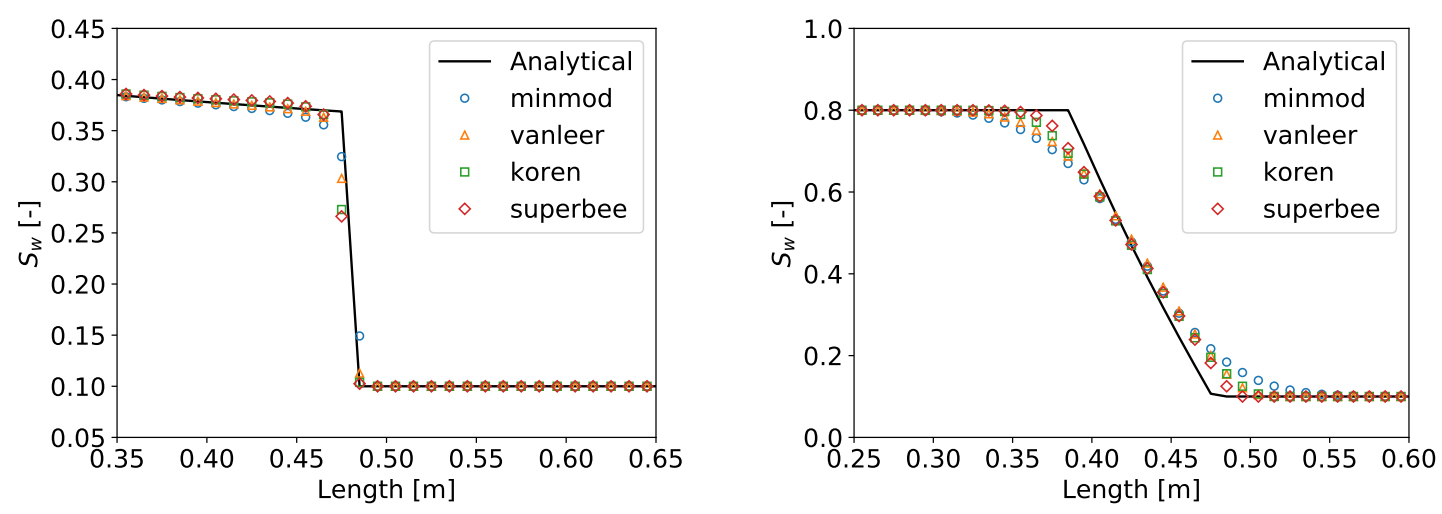

Figure 3.3: Impact of the flux limiter in the solution of the Buckley-Leverett problem. Right: case 1-a, left: case 1-b

\section{Scheme order}

Formally, the spatial discretisation accuracy described in this section is second order but the use of limiters implies that the latter is only true for sufficiently smooth solutions. Richardson extrapolation has been used to estimate the effective scheme order. This method uses the solution computed on three different refined grids with constant refinement factor $r$ [Oberkampf and Roy, 2010]. The order $\hat{p}$ can be estimated with the following formula:

$$
\hat{p}=\frac{\log \left(\frac{\left\|f_{2}-f_{1}\right\|}{\left\|f_{3}-f_{2}\right\|}\right)}{\log (r)},
$$

where $f_{i}$ is the the solution in the coarsest grid points for the $i$ refinement level being $i=1$ the coarsest level and $i=3$ the finest one.

The test has been carried out in 1D problems using 9, 81 and 729 grid points. Refinement factor $r$ for the test is $r=9$. This factor assures the fine grids contain the points of the coarser levels to avoid interpolation. Two different tests based on the Buckey-Leverett solution have been carried out. One with a sharp advancing front and the other with a smooth solution. Solutions are plotted in Figure 3.4 In the smooth case, second order is measured while the scheme order is reduced to one in the presence of a sharp front.

\subsubsection{Boundary conditions}

A wide range of cases of interest in reservoir simulation can be modelled with only 2 boundary conditions: flow (or velocity) and pressure. Walls or impermeable bound- 

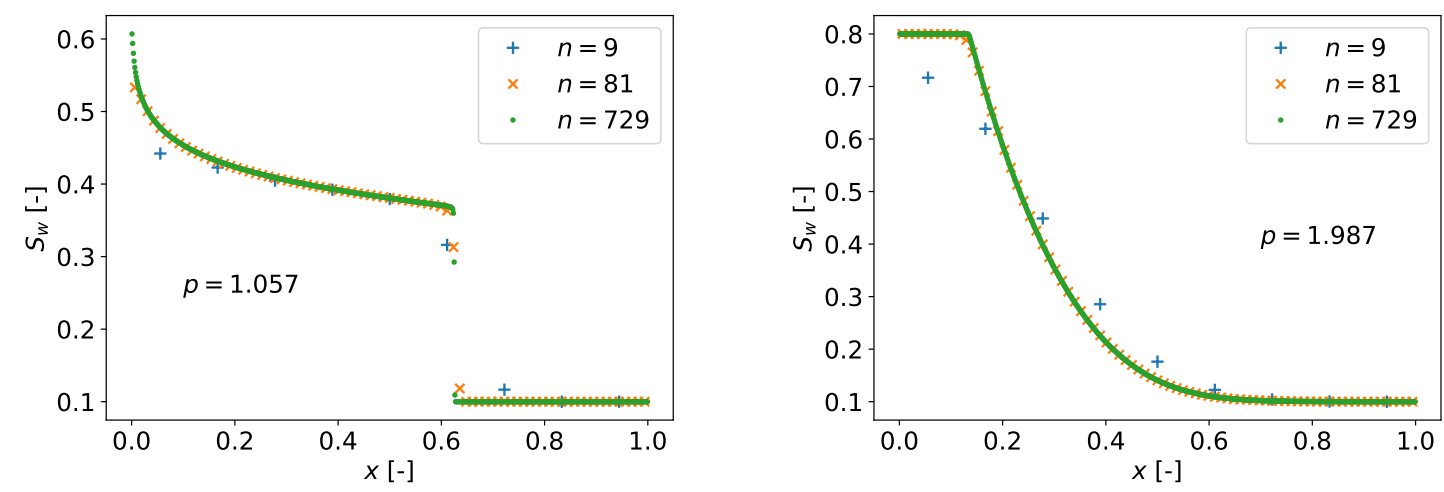

Figure 3.4: Solutions used for scheme order estimation using Richardson extrapolation for a sharp (left) and smooth (right) saturation distribution.

aries can be seen as a velocity condition with zero flow. In addition, a model for including wells is presented.

\section{Pressure inflow / outflow}

Dirichlet boundary condition is imposed to the discretised pressure equation and saturation equations. The row in matrix $A$ in (3.9) is zeroed, 1 added in the main diagonal and the corresponding pressure is imposed in the appropriate location in vector $\boldsymbol{b}$. If the boundary condition corresponds to an inflow boundary, a value for saturation is imposed at the boundary point. If the boundary corresponds to a an outflow, saturation is taken the same as the adjacent cell in normal direction to the boundary.

\section{Flow}

At flow boundary points, the stencil for the pressure discretisation is modified as indicated in Figure 3.5. The total flow is added to the RHS of the system (3.9). In the saturation equation, the wetting phase flux of the boundary points is added to the flux terms in (2.33).

\section{Wells}

In this work, wells are modelled using Peaceman correction [Brekke et al., 1993; Dumkwu et al., 2012; Peaceman, 1978]. This model relates well pressure, cell pressure, and flow. In cells where a well exists, one of those variables is given, the other is known and the third can be calculated using the model. 

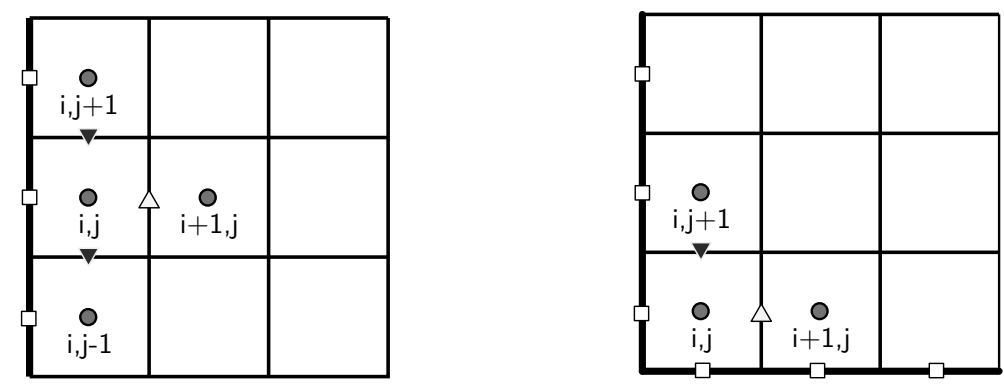

Figure 3.5: 2D stencil used in face boundaries (left) and corners(right). Boundary faces are marked with white squares.

Peaceman model uses the concept of productivity index, $J$, which for a well normal to the domain, is defined as:

$$
J=\frac{2 \pi \sqrt{k_{x} k_{y}}}{\ln \left(\frac{r_{0}}{r_{w}}\right)},
$$

where $k_{x}$ and $k_{y}$ are the absolute permeabilities in $x$ and $y$ direction, $r_{w}$ is the well radius and $r_{0}$ is the equivalent cell radius, defined as:

$$
r_{0}=\frac{0.28 \sqrt{\frac{k_{x}}{k_{y}}(\Delta x)^{2}+\frac{k_{y}}{k_{x}}(\Delta y)^{2}}}{\left(\frac{k_{y}}{k_{x}}\right)^{1 / 4}+\left(\frac{k_{x}}{k_{y}}\right)^{1 / 4}} .
$$

If well pressure is given, injection or production rate for each phase is given by:

$$
q_{i}=J \frac{k_{r, i}}{\mu_{i}}\left(p_{i}-p_{w b}\right), \quad i=w, n,
$$

where $p_{i}$ is the phase pressure in the cell and $p_{w b}$ is the given wellbore pressure.

For a detailed mathematical elaboration for the productivity index $J$ and the equivalent cell radius $r_{0}$, the reader is referred to Peaceman [1978].

On the one hand, if the flow is given for a well, the flow of each phase $q_{\alpha}$ is added to the flow term in equations (2.32) and (2.33), wellbore pressure is computed from equation (3.20) after solving the pressure equation.

On the other hand, if the pressure is given for a well, equation (3.20) is added to the flow terms in (2.32). Once this flux is discretised, a term to the main diagonal of $\boldsymbol{A}$ and another one to the RHS in (3.9) are added. After solving the pressure equation, the flux of each phase in computed with (3.20) and added to the flux terms in the saturation equation (2.33). 


\subsubsection{Mesh refinement}

The numerical discretisation supports the use of non regular cartesian grids. Variable spacing allows local mesh refinement for detailed simulation reducing the size of the problem compared with the use of uniform refinement.
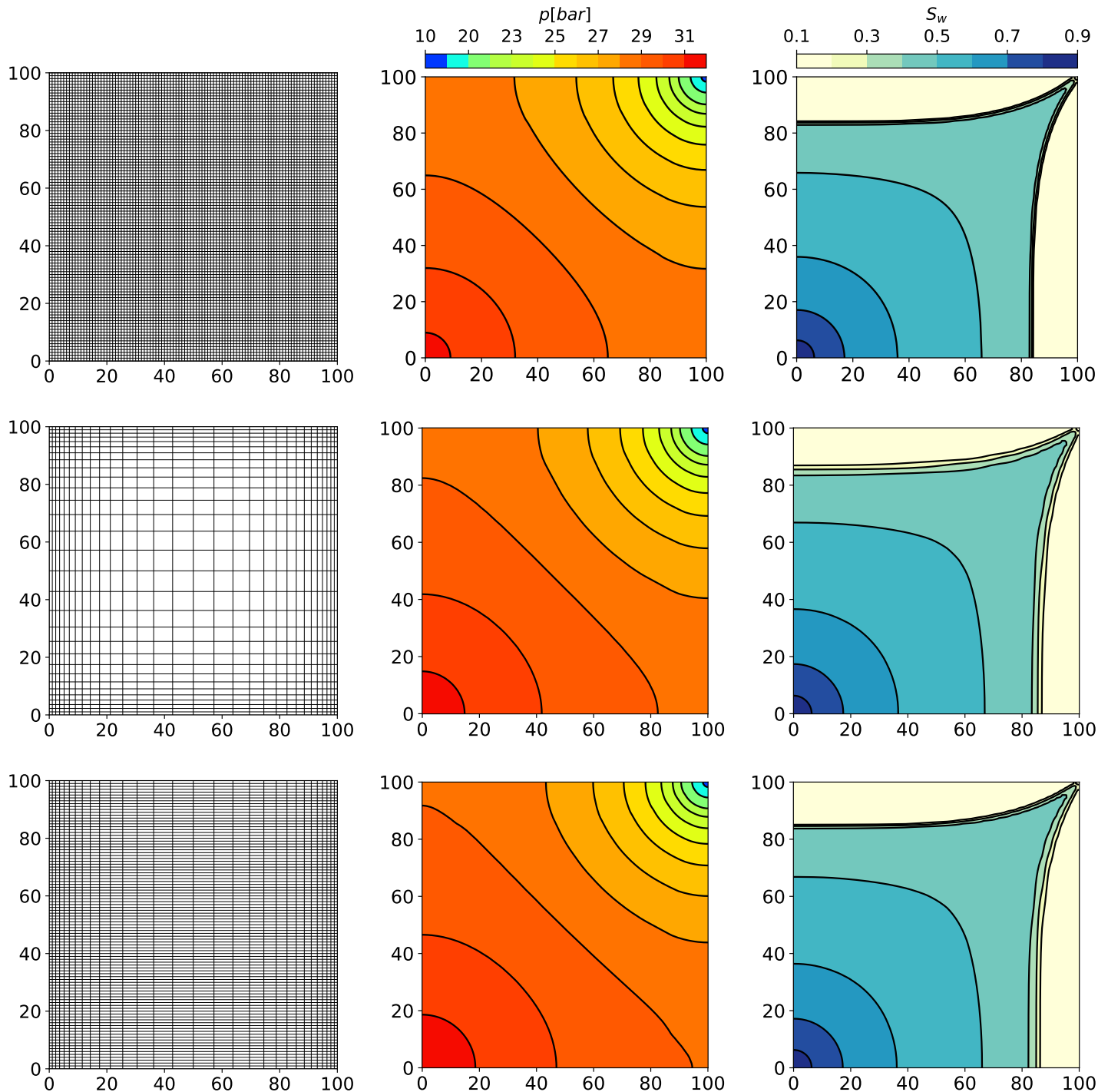

Figure 3.6: Impact of mesh refinement in the solution. Computational mesh (left), pressure (centre) and saturation distribution (right). Grid sizes, from top to bottom: $100 \times 100,30 \times 30$ and $30 \times 100$.

In Figure 3.6 the impact of mesh refinement in the solution is shown. No mesh effect in the solution associated to the refinement, beyond the error associated to spatial resolution, is appreciated. In the case shown in Figure 3.6-bottom the pressure 
and saturation distributions maintain symmetry even with different $x$ and $y$ mesh refinement.

\subsection{Temporal discretisation}

Saturation equation (2.33) is solved using the IMPES method with a RK2 scheme. The discretised algorithm, after introducing (3.7) in (2.33), is listed below:

1. Solve $A^{n} \boldsymbol{p}_{w}^{n}=\boldsymbol{b}^{n}$ and compute $\boldsymbol{u}_{w}^{n}$

2. Compute $\Delta t$

3. $S_{w ; i, j}^{n+1 / 2}=S_{w ; i, j}^{n}+\frac{\Delta t}{2 \phi}\left(q_{w ; i, j}^{n}-\left(\frac{u_{w ; i+1 / 2, j}^{n}-u_{w ; i-1 / 2, j}^{n}}{\left(x_{i+1, j}-x_{i-1, j}\right) / 2}+\frac{u_{w ; i, j+1 / 2}^{n}-u_{w ; i, j-1 / 2}^{n}}{\left(y_{i, j+1}-y_{i, j-1}\right) / 2}\right)\right)$

4. Solve $A^{n+1 / 2} \boldsymbol{p}_{w}^{n+1 / 2}=\boldsymbol{b}^{n+1 / 2}$ and compute $\boldsymbol{u}_{w}^{n+1 / 2}$

5. $S_{w ; i, j}^{n+1}=S_{w ; i, j}^{n}+\frac{\Delta t}{\phi}\left(q_{w ; i, j}^{n}-\left(\frac{u_{w i ; 1 / 2, j}^{n+1 / 2}-u_{w ; i-1 / 2, j}^{n+1 / 2}}{\left(x_{i+1, j}-x_{i-1, j}\right) / 2}+\frac{u_{w ; i, j+1 / 2}^{n+1 / 2}-u_{w, i, j-1 / 2}^{n+1 / 2}}{\left(y_{i, j+1}-y_{i, j-1}\right) / 2}\right)\right)$

Pressure equation (2.32) has to be solved twice each time step. The system of linear equations resulting from the spatial discretisation is solved using an iterative preconditioned GMRES(m) solver [Saad and Schultz, 1986]. This strategy reduces drastically the computational time needed for the implicit part of the IMPES algorithm compared to direct solvers. Iterative pressure solver is discussed in Section 3.3.

Time step computation is based on the stability criteria from Todd et al. [1972] and Coats [2003], adapting it to the staggered grid according to Nessyahu and Tadmor [1990]. Besides, a maximum variation in saturation $\Delta S_{\max }$ per time step is imposed:

$$
\Delta t_{\mathrm{CFL}}=\mathrm{CFL} \cdot \frac{\phi \Delta x \Delta y}{2 \Psi p_{c}^{\prime}\left(T_{x}+T_{y}\right)+2\left(\left|f_{w}^{\prime} \hat{q}_{x}\right|+\left|f_{w}^{\prime} \hat{q}_{y}\right|\right) \Delta x \Delta y},
$$

where

$$
\begin{gathered}
\Psi=\frac{\lambda_{w} \lambda_{n}}{\lambda_{n}+\lambda_{w}}, \\
p_{c}^{\prime}=\frac{\mathrm{d} p_{c}}{\mathrm{~d} S_{w}},
\end{gathered}
$$

$T$ are the transmissibilities defined as:

$$
\begin{aligned}
T_{x, i j} & =\left(\frac{k_{x, i-1 / 2, j}}{x_{i}-x_{i-1, j}}+\frac{k_{x, i+1 / 2, j}}{x_{i+1}-x_{i}}\right) \Delta y, \\
T_{y, i j} & =\left(\frac{k_{y, i, j-1 / 2}}{y_{j}-y_{j-1}}+\frac{k_{y, i, j+1 / 2}}{y_{j+1}-y_{j}}\right) \Delta x,
\end{aligned}
$$


$f_{w}^{\prime}=\mathrm{d} f_{w} / \mathrm{d} S_{w}$ is the derivative of the fractional flow $f_{w}=\lambda_{w} /\left(\lambda_{w}+\lambda_{n}\right)$ and $\hat{q}_{i}$ is the maximum total flow on each direction. The term $f_{w}^{\prime} \hat{q}_{x}$ is computed from face variables and is defined as:

$$
\begin{aligned}
f_{w}^{\prime} \hat{q}_{x, i j} & =\max \left(\frac{f_{w, i+1, j}^{\prime}+f_{w, i, j}^{\prime}}{2}\left|u_{w, i+1 / 2, j}+u_{n, i+1 / 2, j}\right|,\right. \\
& \left.\frac{f_{w, i-1, j}^{\prime}+f_{w, i, j}^{\prime}}{2}\left|u_{w, i-1 / 2, j}+u_{n, i-1 / 2, j}\right|\right) .
\end{aligned}
$$

The solution is stable and free of oscillations for CFL $<1$ and stable for CFL $<2$ [Coats, 2003]. The additional time step restriction is defined:

$$
\Delta t_{\Delta S_{\max }}=\frac{\Delta S_{\max } \phi}{q_{w}-\nabla \cdot \boldsymbol{u}_{w}}
$$

The time step restriction associated to maximum saturation change is needed to avoid sudden changes in cell saturation that can affect the convergence of the iterative solver, for example, in the presence of wellbores. After several tests, value of $\Delta S_{\max }=$ 0.1 has been chosen. This criteria is more restrictive than the CFL only for the first iterations in wellbore simulations or highly inhomogeneous domains.

Iteration time step is the minimum value among all the points in the domain for both criteria:

$$
\Delta t=\min \left(\Delta t_{\mathrm{CFL}}, \Delta t_{\Delta S_{\max }}\right)
$$

\subsection{Iterative pressure solver}

As it was stated in the previous section, IMPES strategy for solving system (2.32) -(2.33) requires two resolutions of a sparse linear system associated to the discretisation of pressure equation each iteration. In classical IMPES implementations, this phase takes the major part of the computational time. Thus the overall performance of the method relies on the efficiency of the pressure equation resolution. This system can be solved using direct methods, which are robust and compute the solution up to machine precision. However they are expensive from the point of view of computational time and present high memory requirements.

Iterative solvers are the alternative to direct ones. One of the advantages of these methods is that the solution can be approximated up to a desired tolerance. Iterative methods are much less robust than direct ones and the efficiency relies on the initial guess and the spectrum of the coefficients matrix (which are problem dependent) and 
the desired tolerance of the solution. For a thorough review of iterative methods refer to Saad [2003] and Barrett et al. [1994].

The task of the pressure solver can be seen as a correction of the pressure field between iterations up to a desired tolerance. The explicit time step is restricted by the stability criteria so small pressure changes are expected between consecutive time steps. This fact can be exploited by iterative methods.

The term iterative method refers to a wide range of techniques that use successive approximations to obtain more accurate solutions to a linear system at each step. Stationary methods are older, simpler to understand and implement, but usually not as effective. Some of the more used stationary methods are the Jacobi , the Gauss-Seidel, the Successive Overrelaxation (SOR) and the Symmetric Successive Overrelaxation (SSOR) method. Nonstationary methods are harder to understand and implement, but they can be highly effective. The nonstationary methods are based on the idea of sequences of orthogonal vectors. Some of the most used are the Conjugate Gradient (CG), the BiConjugate Gradient Stabilised (Bi-CGSTAB) and the Generalised Minimal Redisual (GMRES). The rate at which an iterative method converges depends greatly on the spectrum of the coefficient matrix [Barrett et al., 1994]. Hence, iterative methods usually involve a second matrix that transforms the coefficient matrix into one with a more favorable spectrum. The transformation matrix is called preconditioner. A preconditioner has to sufficiently improve the convergence to overcome the extra cost of computing and applying it.

Coefficient matrix of system (3.29) is structurally symmetric but numerically unsymmetric and can be extremely ill conditioned, the matrix is nearly numerically singular when a wide range of permeabilities is present. In this context GMRES can converge safely to the solution (or the least-squares solution if the matrix is singular) if an appropriate initial condition is used [Brown and Walker, 1997]. Other methods for unsymmetric matrices such as stationary or Bi-CGSTAB can stagnate or crash for (nearly) numerically singular coefficient matrices. For this reason, in this work a GMRES based method named restarted Flexible Generalized Minimum Residual (FGM$\operatorname{RES}(\mathrm{m})$ ) is used.

\subsubsection{FGMRES(m)}

Flexible GMRES (FGMRES) is a variation of GMRES where the preconditioner may vary between iterations. This flexibility allows the use of iterative techniques as preconditioners in addition to fixed matrices [Saad, 2003]. The pseudocode of the method is listed in Algorithm 1.

GMRES iteration is restarted, using the last approximation of the solution as initial guess every $m$ iterations. The reason is that the computational cost and memory 


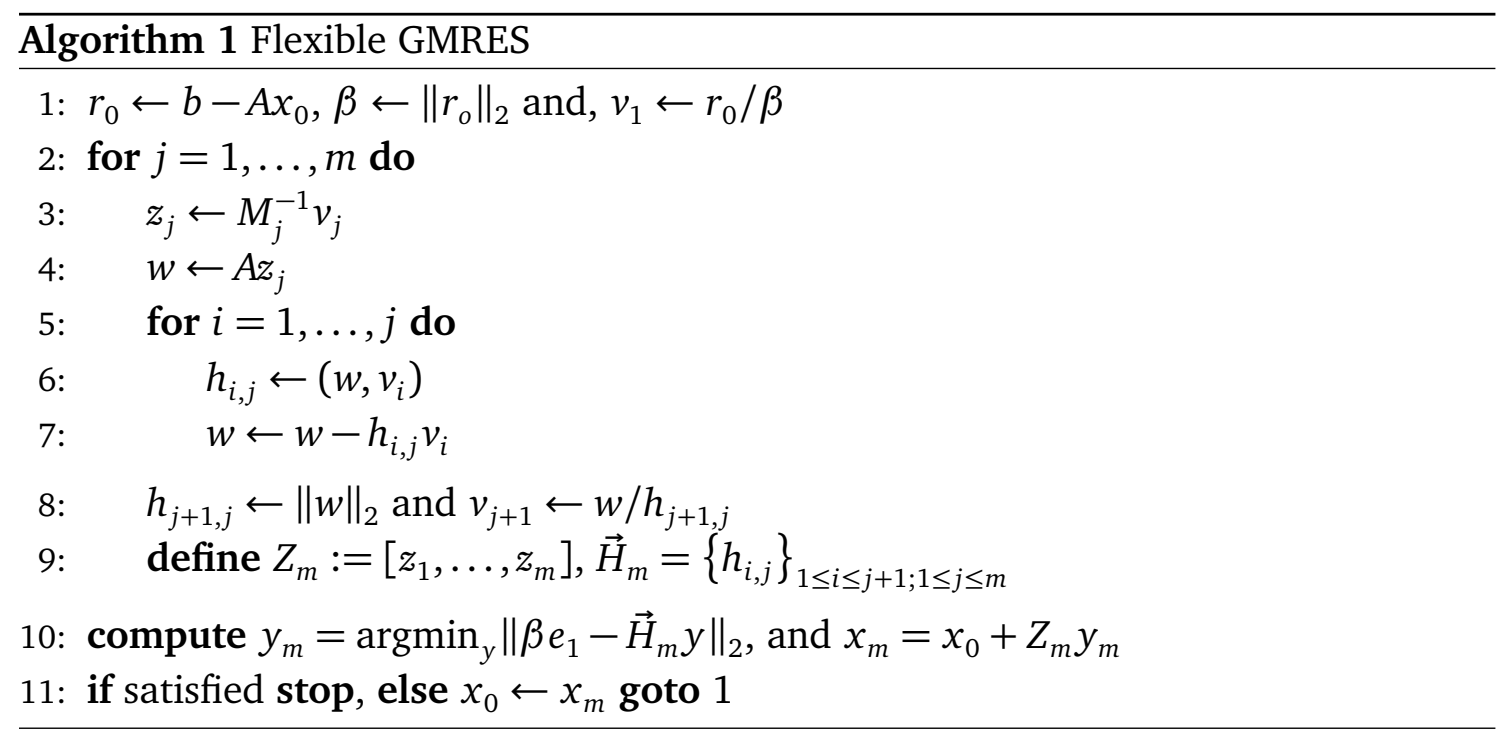

requirements grow fast as the number of iterations increases. The choice of a small value of $m$ increases the speed of the iterations but can stagnate the convergence thus, a proper value of $m$ has to be chosen carefully as a compromise solution between performance and robustness. In Section 4.2.2, it is shown that small values of $m$ can easily break the convergence for the matrices arising from the pressure equation (2.19).

The explicit time step restriction imposed by the stability criteria is exploited to improve the performance of the iterative solver. Proper initial guess affects the convergence of iterative solvers, especially if ill-conditioned matrices are involved. The pressure calculated in the previous time steps can be used to compute an efficient estimation for the initial guess.

\section{Preconditioners}

Preconditioners are used with iterative solvers to transform an ill-conditioned linear system,

$$
A x=b,
$$

into the equivalent,

$$
M^{-1} A x=M^{-1} b
$$

$M$ is chosen such that the matrix $\hat{A}=M^{-1} A$ is better conditioned for the iterative solver than the original one. For the case of GMRES, $A$ can be effectively precondicioned, clustering the eigenvalues around the origin, using an approximation to the inverse of the matrix [Saad, 2003]. In Algorithm 1, the preconditioning operation is performed in line 3 . 
In this work, three different preconditioners are tested: ILU(0), ILUT and SSOR. The three preconditioners are computed in LU form:

$$
M=L U,
$$

where $L$ is lower triangular and $U$ upper triangular. With this factorisation, the preconditioning operation:

$$
z=M^{-1} v
$$

can be implemented by decomposing it in two triangular systems:

$$
\begin{aligned}
& L x=v, \\
& U z=x,
\end{aligned}
$$

that can be solved efficiently by substitution.

ILU(0) This preconditioner belongs to the category of incomplete LU factorization, which is derived from Gauss elimination, dropping elements out of the main diagonal [Saad, 2003]. ILU(0) takes the same non-zero pattern $(N Z(A))$ as the original matrix. A common algorithm for computing the ILU(0) matrix reads:

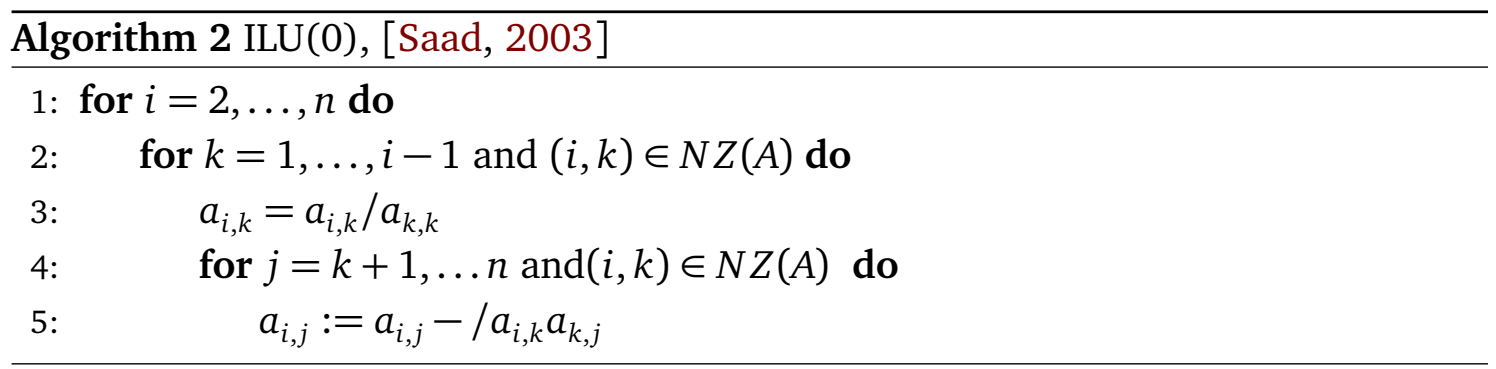

The general matrix form form for the ILU(0) preconditioner is:

$$
M_{I L U 0}=\left(I-E D^{-1}\right)(D-F),
$$

where $E$ is the strictly lower part of $A, F$ is the strictly upper part of $A$ and $D$ is a diagonal matrix.

ILUT This variation of incomplete LU factorization does not conserve elements by position but for magnitude. The method, known as $\operatorname{ILUT}(p, \tau)$, conserves the $p$ values with higher magnitude in a row, for the upper and lower matrices, that are above a certain threshold $\tau$. The algorithm to compute this factorization is listed below: 


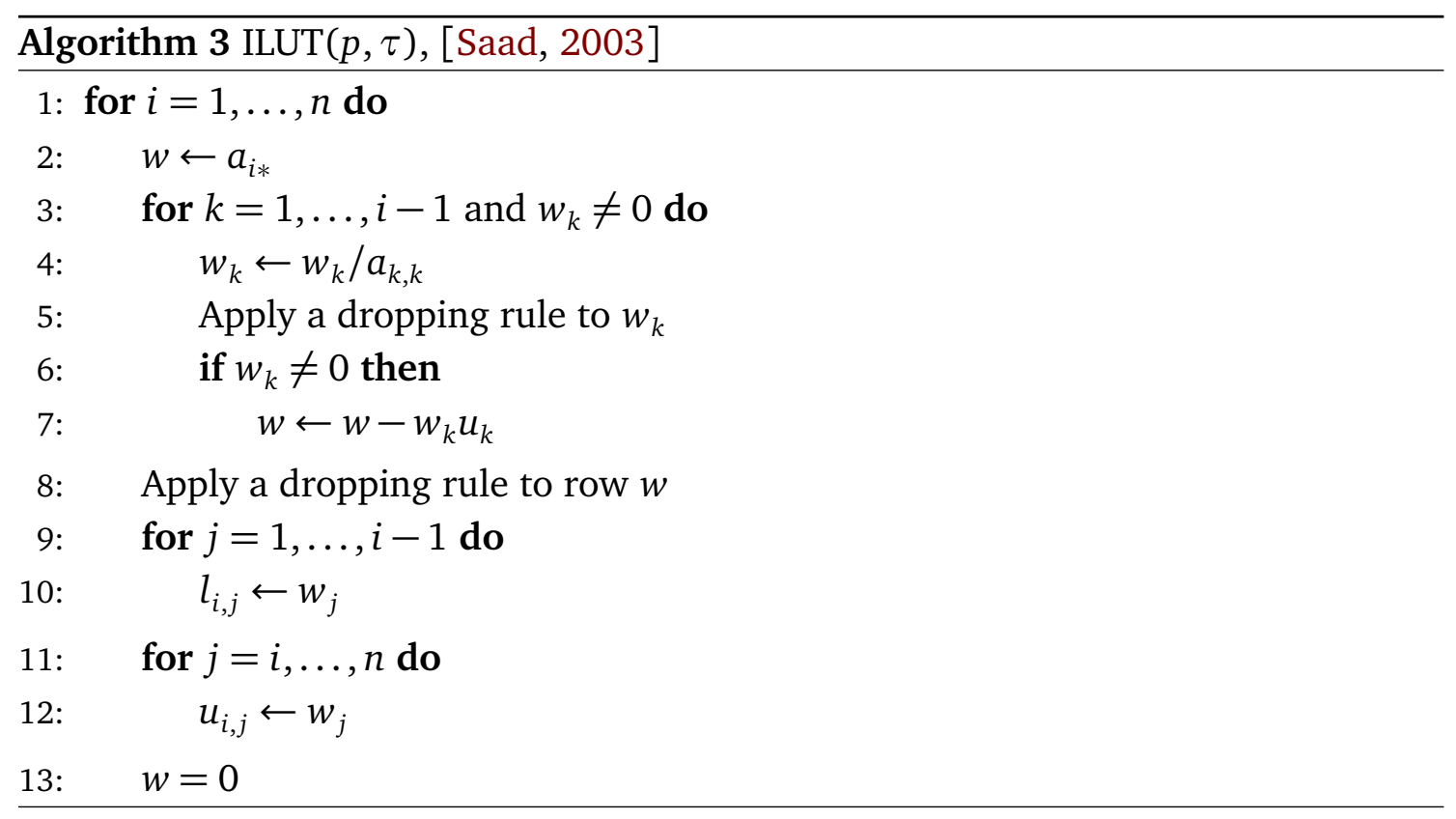

In line 5 , an element $w_{k}$ is replaced with zero if it is below the relative tolerance $\tau$ multiplied by the norm of the $i$-th row. In line 8 only the $p$ largest elements in the $\mathrm{L}$ and the $\mathrm{U}$ part of the row are kept.

SSOR This preconditioner is derived from the iterative method symmetric successive over relaxation, a variant of Gauss-Seidel algorithm, for solving linear systems. The matrix form of the preconditioner is:

$$
M_{S S O R}=(D-\omega E) D^{-1}(D-\omega F)=\left(I-\omega E D^{-1}\right)(D-\omega F)=L U,
$$

where $\omega$ is a parameter between 1 and 2. $E$ is the strictly lover part of $A, F$ the strictly upper part and $D$ the main diagonal of $A$. According to Saad [2003] the parameter $w$ has little influence on the efficiency of the preconditioner.

In Section 4.2.1 the efficiency of the three preconditioners is compared for different simulations.

\subsubsection{Iterative solver tolerance}

GMRES method needs a stopping criteria to skip the iterative process when a desired tolerance in the error of the solution has been reached. The norm of the residual of (3.9) can be used,

$$
r=\left\|\boldsymbol{A} \hat{\boldsymbol{p}}^{k}-\boldsymbol{b}\right\|
$$


where $\hat{\boldsymbol{p}}^{k}$ is the approximation of the solution in the $k$-th GMRES iteration. This norm requires an extra matrix product each iteration. Besides, the norm is related to the pressure field and the relation between the error in the pressure and saturation distributions is problem dependent. The GMRES residual $h_{j+1, i}$ (line 8 Algorithm 1 ) can be used to monitor the GMRES convergence but the relation with the error in pressure or saturation is unknown a priori and is problem dependent. The error in the saturation field associated with the error in the iterative solver can be estimated using the error in divergence of total velocity per time step:

$$
\operatorname{DIV}_{\text {error }}=\max \left[\nabla \cdot\left(\left.\boldsymbol{u}_{w}\right|_{i, j}+\left.\boldsymbol{u}_{n}\right|_{i, j}\right)+\left.q_{w}\right|_{i, j}+\left.q_{n}\right|_{i, j}\right] \Delta t
$$

Equation (3.38) is zero when the pressure equation is solved exactly. It should be mentioned that the computation of this control variable involves a negligible cost because all the involved variables are calculated during the saturation update phase.

In Figure 3.7 the convergence history for GMRES residual $h_{j+1, i}$, pressure residual $r$ from (3.37) and divergence tolerance DIV $_{\text {tol }}$ against the number of GMRES iterations are plotted. These figures have been computed solving the same time step with different number of GMRES iterations and saving GMRES tolerance, the error in pressure and the error in total velocity divergence after each GMRES loop.
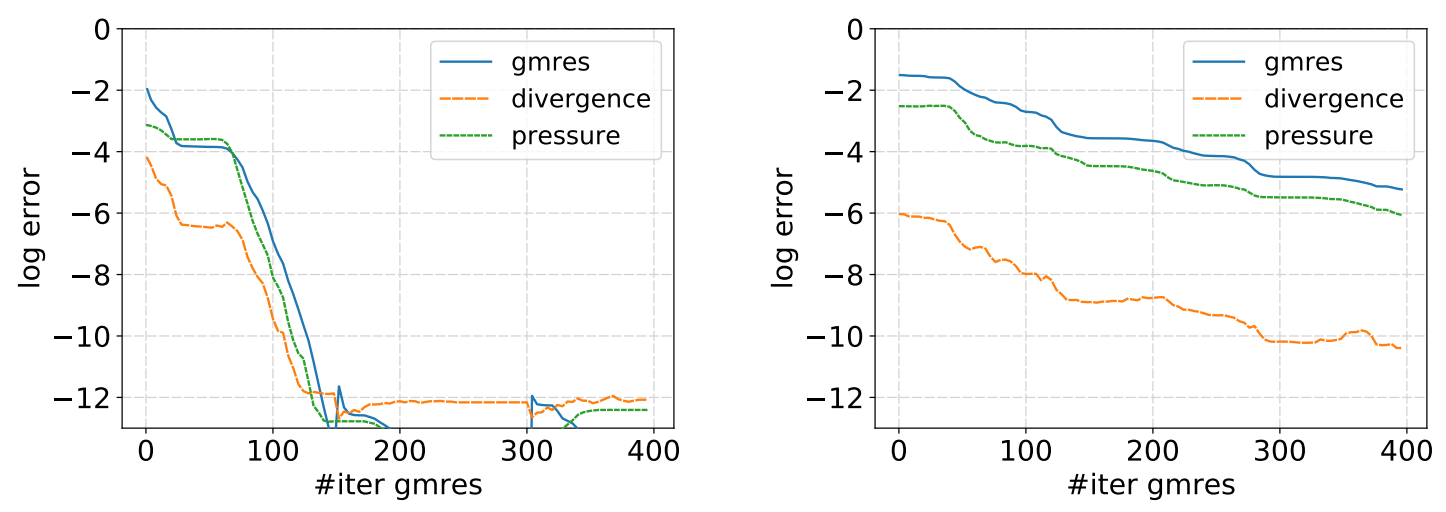

Figure 3.7: Evolution of the GMRES tolerance, pressure error and total divergence error with the number of GMRES iterations for a front advancing (left) and five-spot problem.

The relation between DIV $_{\text {tol }}$ and GMRES tolerance is not known and is problem dependent but, as can be drawn from Figure 3.7 both tolerances have the same behaviour and, as a result, the error in saturation can be controlled by adapting the error in GMRES. For that purpose, a simple linear control algorithm is implemented to correct the 
GMRES tolerance every time step to obtain a desired error in total divergence:

$$
\text { GMRES-TOL }^{k+1}=\text { GMRES-TOL }^{k} \frac{\tau}{\mathrm{DIV}_{\text {error }}^{k}},
$$

where $\tau$ is the desired tolerance in total velocity divergence per time step and $\mathrm{DIV}_{\text {error }}^{k}$ is the divergence error in the previous iteration $k$.

As an example, in Figure 3.8, the error in total divergence and the GMRES tolerance are plotted for the five-spot validation case (see 4.1.5). In the plot can be observed that the GMRES tolerance is modified during the simulation to maintain the desired tolerance in the total velocity divergence. Despite showing some oscillations, the simple control algorithm is able to track the desired divergence tolerance accurately. GMRES tolerance can vary several order of magnitude during a simulation due to changes in the pressure distribution and variations in the time step size.

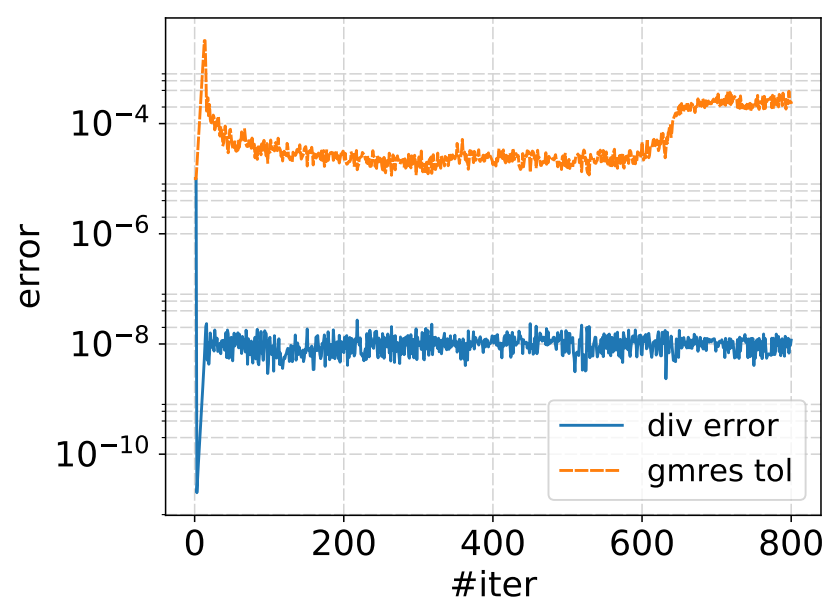

Figure 3.8: Evolution of GMRES tolerance and error in total velocity divergence per time step for test case 5 (see 4.1.5). Target divergence error is set to $10^{-8}$.

In the following chapter the impact of the linear solver tolerance in both the efficiency of the iterative solver strategy and the error in the solution is widely analysed. 


\section{chapter 4}

\section{NUMERICAL EXPERIMENTS AND VALIDATION}

\subsection{Validation cases}

In this work, six simulations have been used for validation and to show the accuracy, robustness and efficiency of the discretisation presented in Chapter 3. These test cases have been selected because they represent a wide spectrum of physical problems of interest. In particular, special attention is paid to gravity, capillary pressure and localised high flow velocity effects (both geometrically and permeability induced). All the cases presented in this section have been extracted from published works for validation purpose. In all the 2D cases, the impact of the GMRES preconditioner in the efficiency of the method is studied as well as the impact of the solver tolerance both in efficiency and solution accuracy. Solutions with different tolerances are compared with a reference solution computed with the IMPES method and an exact linear solver.

Case 1 is the solution of the Buckley-Leverett equation [Buckley and Leverett, 1942]. This case is useful for validation since an analytical solution exits for the equation, allowing to compare numerical results with the analytical solution. This case is the one used to analyse the effect of different flux limiters in the numerical results in Chapter 3.

Case 2 is extracted from the 2001 SPE Comparative Solution Project [Christie and Blunt, 2001], organised by the Society of Petroleum Engineers. The purpose of the project was to provide benchmark datasets which can be used to compare the performance of different simulators or algorithms. The selected case is the dataset 1 , a $2 \mathrm{D}$ configuration with gravity effects and a highly heterogeneous permeability distribution. Results are compared with the work of Franc et al. [2016].

Case 3 is a banded domain from [Hoteit and Firoozabadi, 2008; Yang et al., 2016] 
with capillary effects. This case is used to test and validate the capillary pressure model. Also the efficiency of the method presented in this work is compared to the results from Hoteit and Firoozabadi [2008] and Yang et al. [2016].

Case 4 is a front advancing problem in a domain with different permeability zones with no gravity and capillary pressure. In particular, 3 different permeability distribution are simulated to validate the results with [Yang et al., 2016].

Case 5 is a $2 \mathrm{D}$ five-spot simulation on a random domain, in this case the effect of well production oscillation associated to IMPES method is analysed as well as the effect of solver tolerance in the accuracy of the results and computational time.

Case 6 is a 3D configuration from 2001 SPE-10 Comparative Solution Project [Christie and Blunt, 2001] dataset 2. The model is defined on a grid of $60 \times 220 \times 85$, approximately 1.1 million cells. The model is a five-spot configuration with one injector and four producer wells. Permeability and porosity are highly heterogeneous, including death cells with null permeability. This case is used to test the accuracy and robustness of the the algorithm and the 3D implementation on a long run simulation.

All the results involving timing or efficiencies have been computed on a 2x Intel(R) Xeon(R) CPU E5-2630 @ 2.30GHz workstation with a total of 12 physical cores using 4 threads.

\subsubsection{Case 1. Buckley-Leverett problem.}

In this case, the wetting phase is injected from the left into an uniform domain saturated with nonwetting phase. In the right side of the domain, a fixed pressure condition is imposed. The flow is 1D and can be described using the Buckley-Leverett solution [Buckley and Leverett, 1942]. Three variations of the case have been tested with different parameters using the modified (2 exponents) Brooks-Corey model for the relative permeability. Parameters are listed in Table 4.1. Both capillary and gravity effects are neglected.

Table 4.1: Brooks-Corey relative permeability parameters for the case 1.

\begin{tabular}{lccc}
\hline & Case 1a & Case 1b & Case 1c \\
\hline$n$ & 6 & 1 & 1 \\
$m$ & 3 & 1 & 1 \\
$S_{r, 1}$ & 0.2 & 0.2 & 0.2 \\
$S_{r, 2}$ & 0.1 & 0.1 & 0.1 \\
$k_{r, 1}$ & 0.9 & 0.9 & 0.9 \\
$k_{r, 2}$ & 0.5 & 0.5 & 0.5 \\
$\mu_{1} / \mu_{2}$ & 10 & 2 & 1 \\
\hline
\end{tabular}


Analytical and numerical solutions are compared in Figure 4.1. Volume of water injected divided by the total pore volume or Pore Volume Injected (PVI) is used as non dimensional time. The second order TVD scheme used captures accurately sharp fronts, associated to non linear permeabilities (Case 1a) or uniform displacement (Case 1c), and shows low numerical diffusion on smooth fronts associated to linear permeabilities (Case 1b). Results are plotted in Figure 4.1. As can be seen in the figures, position and height of the fronts are accurately captured with low diffusion effects. This feature is important for predicting instabilities like viscous fingering [Mcdowell et al., 2016; Pavone, 1992; Riaz and Meiburg, 2003] or other physical models like phase changes [Shi et al., 2015], chemical reactions [Hornung and Jäger, 1991] or compositional simulations [Pourafshary et al., 2009; Panfilov et al., 2012].
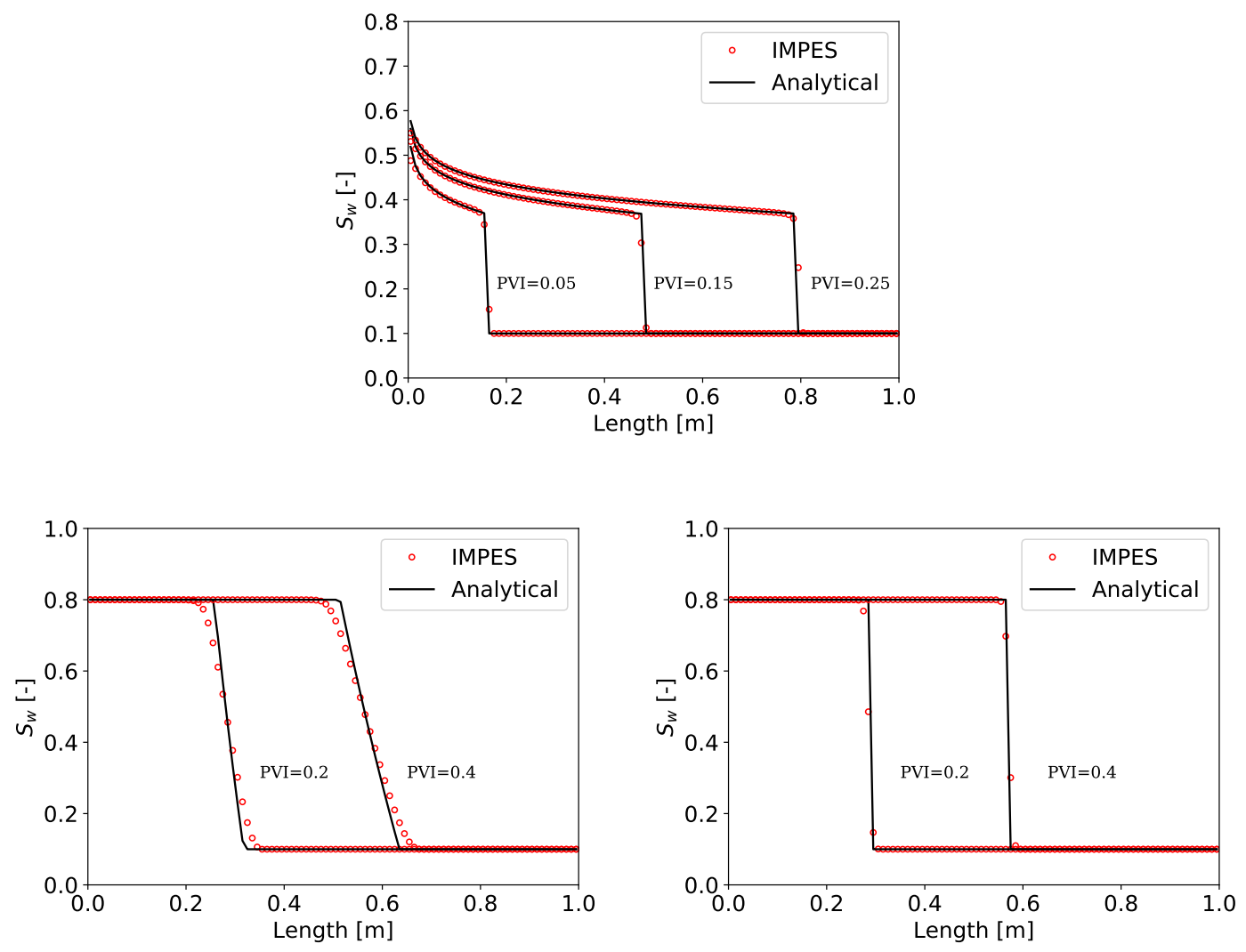

Figure 4.1: Water saturation profiles for the Buckley-Leverett solution for different pore volume injected for the cases $1 \mathrm{a}$ (top), 1b (left), 1c (right) 


\subsubsection{Case 2. SPE10 dataset 1}

This case is part of the SPE10 Comparative Solution Project, the dataset 1 consist of a 2D two phase (oil-gas) model with a simple 2D vertical section geometry [Franc et al., 2016; Christie and Blunt, 2001]. Gas is injected from an injector located on the left of the model and dead oil is produced from a well on the right of the model. Relative permeability parameters and fluid properties are taken from [Franc et al., 2016] and listed in Table 4.2 .

Table 4.2: Case 2 parameters.

\begin{tabular}{ccccccccc}
\hline$n$ & $m$ & $S_{r, 1}$ & $S_{r, 2}$ & $k_{r, 1}$ & $k_{r, 2}$ & $\rho_{1}\left[\mathrm{~kg} / \mathrm{m}^{3}\right]$ & $\rho_{2}\left[\mathrm{~kg} / \mathrm{m}^{3}\right]$ & $\boldsymbol{g}\left[\mathrm{m} / \mathrm{s}^{2}\right]$ \\
\hline 5 & 5 & 0.1 & 0.25 & 1. & 1. & 1. & 700. & $(0 .,-9.8)$ \\
\hline
\end{tabular}

Domain size is $762 \times 15.24 \mathrm{~m}^{2}$ and the mesh resolution is $100 \times 20$. Porosity is uniform with a value $\phi=0.2$. Permeability is isotropic, the distribution is shown in Figure 4.2. The domain presents horizontal bands of high permeability. The ratio between the maximal and the minimal absolute permeability is $10^{6}$. For the simulations shown in Figure 4.2, IMPES with direct (exact) solver has been used. Solutions are in good agreement with the results of Franc et al. [2016].

Residual saturation for gas phase is $S_{n, r}=0.1$ and for oil $S_{w, r}=0.25$. Initially, the media is fully saturated with oil.

The impact of the tolerance in the saturation distribution is shown in Figure 4.3 for the simulation with gravity effects. As can be observed, tolerance values of $10^{-3}$ and $10^{-4}$ lead to unphysical values in saturation with errors of the same order of magnitude of the solution. Tolerance values of the order of $10^{-6}$ are required in this case to assure physical values in the saturation distribution.

Gravity has an accumulation effect in the error in zones with gravity driven flow thus a lower tolerance is required to obtain accurate results. This effect can be noticed after comparing the error plots in the simulation with gravity in Figure 4.3 and the simulation with no gravity in Figure 4.4.

In Figure 4.5 the gas cut (gas flow referenced to the total flow) produced in the producer well, on the right boundary of the domain, is used to measure the impact of the tolerance value. Associated with the error in saturation distribution, tolerance of $10^{-4}$ leads to high error in flow predictions while a tolerance of $10^{-5}$ produce a close approximation to the distribution computed with the direct linear solver. Tolerances of $10^{-6}$ and below predict distributions indistinguishable from the one computed with direct linear solver.

Increasing the tolerance has a negative impact in the solution accuracy, although it can decrease the computational time required by the linear solver and thus the overall 


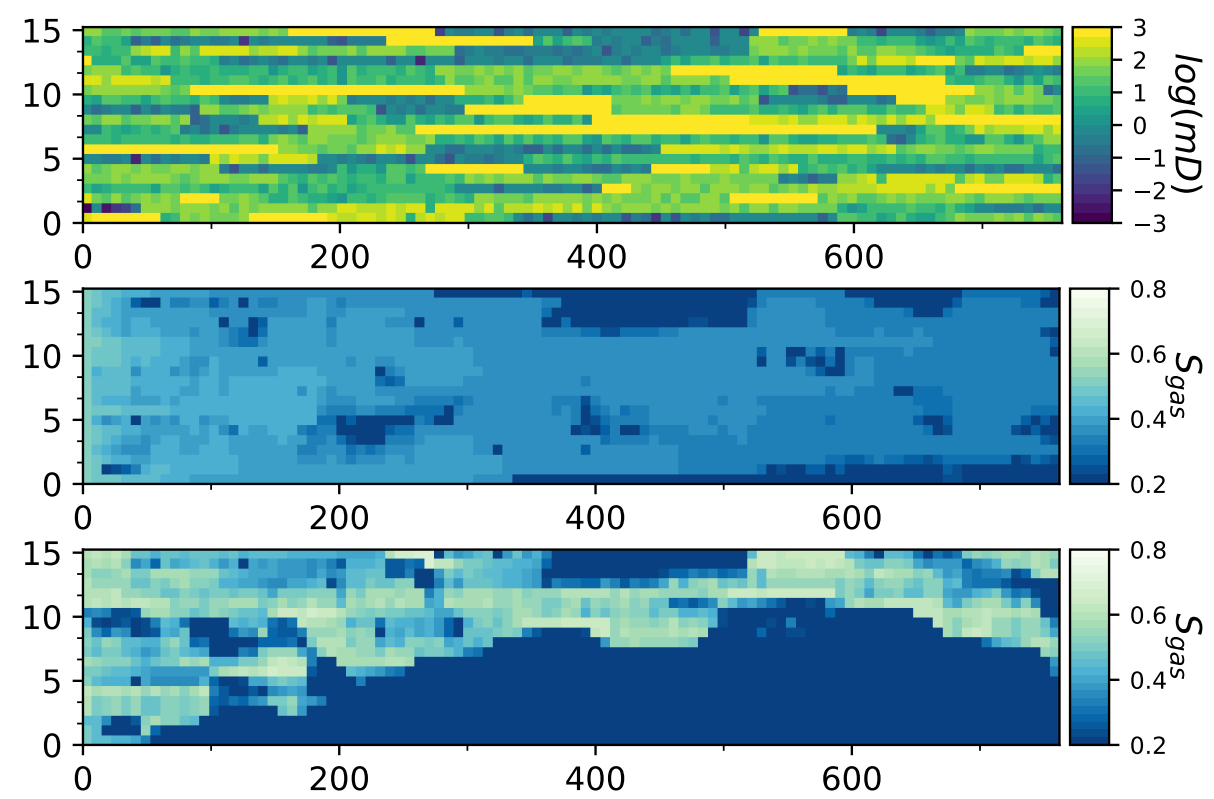

Figure 4.2: Case 2. Top: Absolute permeability field. Centre: gas saturation distribution with no gravity effects. Bottom: gas saturation distribution with gravity effects. Solution after 3 years with uniform gas injection rate at $6.6 \cdot 10^{-7} \mathrm{~m} / \mathrm{s}$ on the left boundary. Domain dimensions in meters ( $y$ dimension scaled 1:10 for visualization).
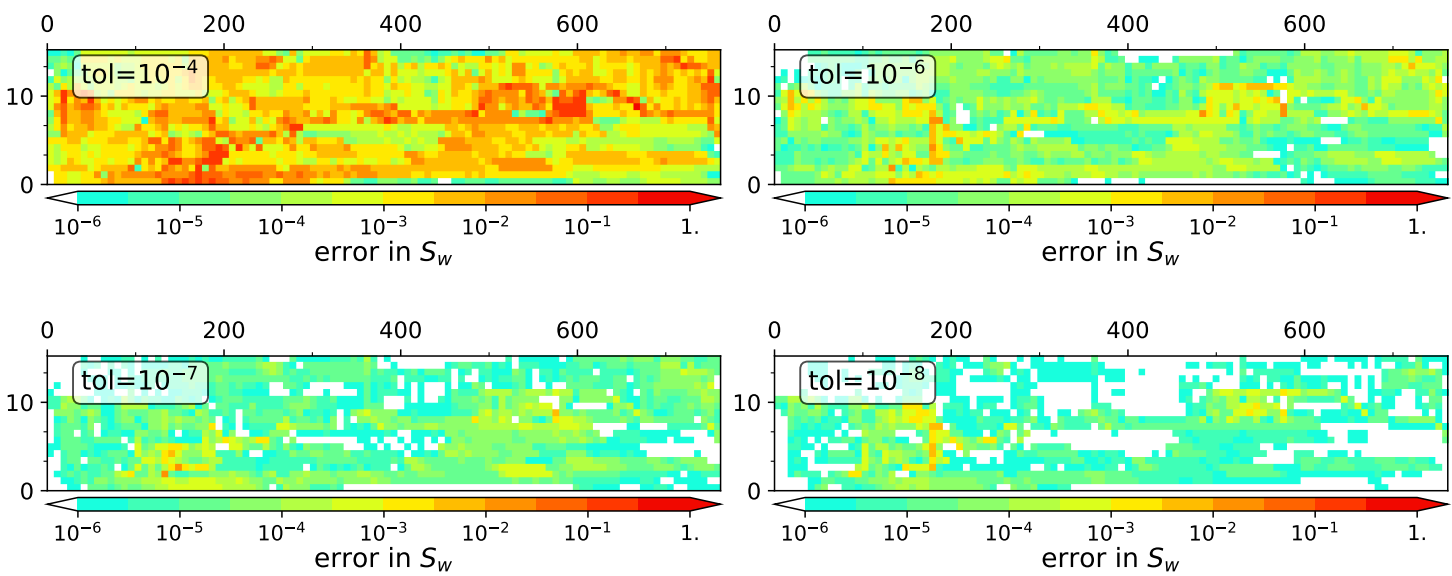

Figure 4.3: Error in saturation distribution after 3 years for case 2 with gravity effects for different tolerance values.

computational time. In Figure 4.6, the reduction of the computational cost with the tolerance is shown. The efficiency of the solver, defined as the inverse of simulation time with a certain tolerance referenced to the simulation time with the exact solver, is plotted using ILU(0) as preconditioner. In the simulation with no gravity, efficiency values of 10 have been measured, for a tolerance of $10^{-5}$ where the results were close 

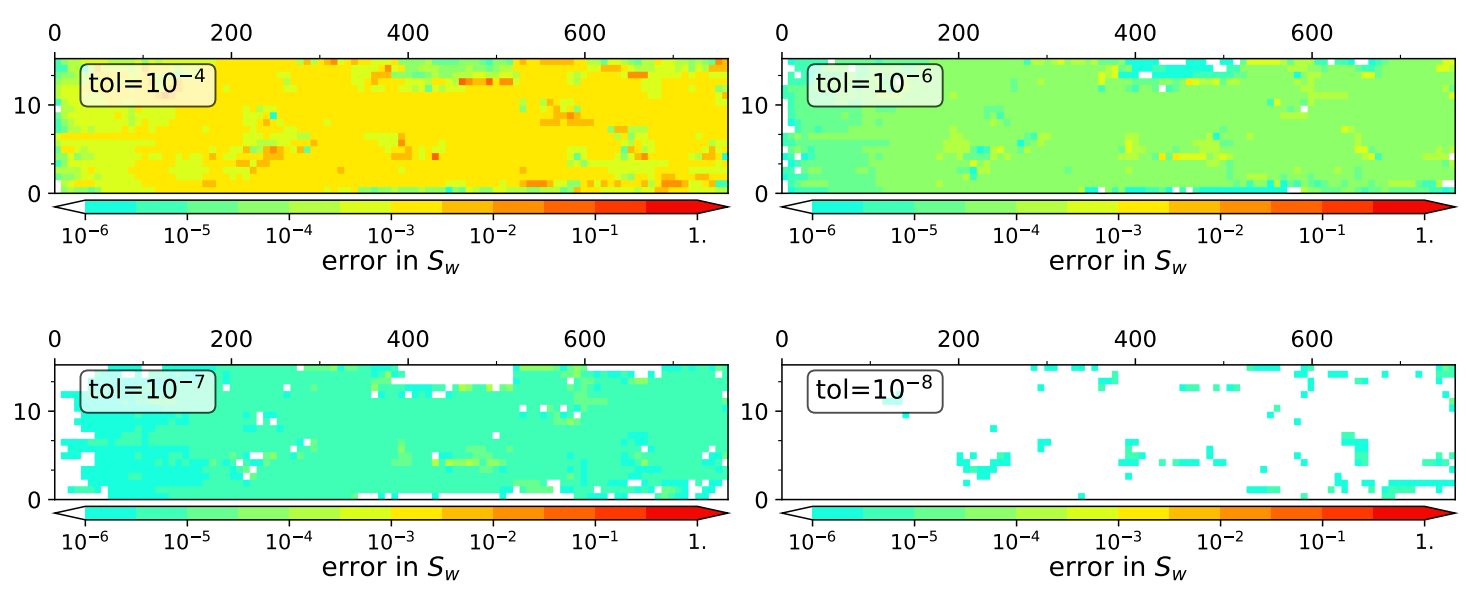

Figure 4.4: Error in saturation distribution after 3 years for case 2 without gravity effects for different tolerance values.
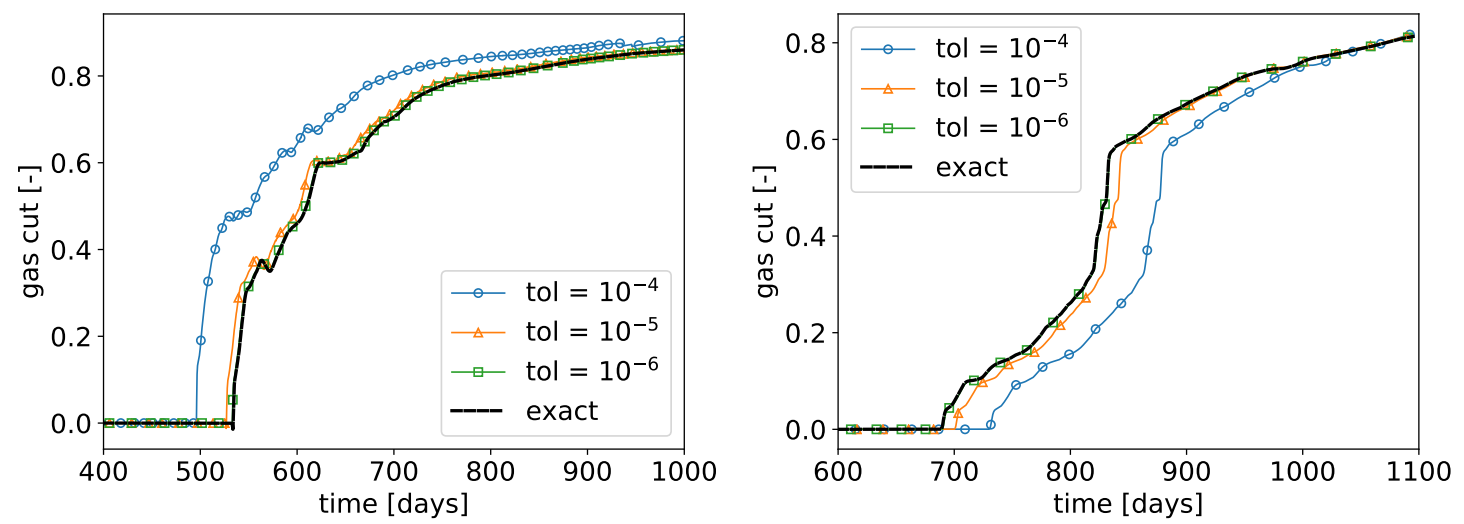

Figure 4.5: Gas production relative to total production in the producer well on the right boundary in case 2 with gravity effect (left) and without gravity effect (right). Markers every 400 time steps.

to the results computed with the exact solver. For a tolerance of $10^{-6}$ where results where indistinguishable from the ones computed with the exact solver, the efficiency is 7. In the simulation with gravity similar efficiency values have been measured. In Table 4.3 the simulation times for the exact solver and the iterative one are shown.

Gravity with complex permeability distribution may produce an accumulation effect in the error as can be observed in Figures 4.3 and 4.4 but, from the analysis of gas production with respect total production (gas cut) curves (Figure 4.5), this effect has almost no impact in global results e.g., in flow production. 


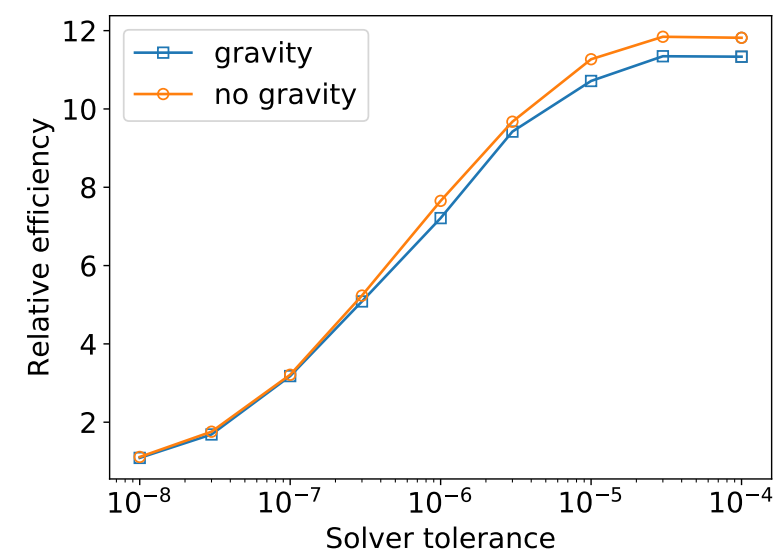

Figure 4.6: Efficiency of iterative solver for case 2 with ILU(0) as preconditioner.

Table 4.3: Computational times for case 2 with and without gravity, $p$ corresponds to the pressure and $S$ to the saturation solver computational time.

\begin{tabular}{rrrr|rrr|rr}
\multicolumn{4}{c}{ IMPES - Exact } & \multicolumn{1}{c}{ IMPES - tol= $10^{-6}$} \\
\hline & time $(\mathrm{s})$ & $p(\mathrm{~s})$ & $S(\mathrm{~s})$ & time $(\mathrm{s})$ & $p(\mathrm{~s})$ & $S(\mathrm{~s})$ & \#Iter & speedup \\
\hline Gravity & 138 & 133 & 3.2 & 19 & 14 & 3.2 & 8426 & 7.3 \\
No gravity & 142 & 137 & 3.2 & 19 & 15 & 3.2 & 8426 & 7.4 \\
\hline
\end{tabular}

\subsubsection{Case 3. Banded plug}

This case is extracted form the works of Hoteit and Firoozabadi [2008] and Yang et al. [2016]. We consider a 2D heterogeneous domain composed of horizontal layers with alternate absolute permeability oriented along the main flow direction, permeability distribution is shown in Figure 4.7. The simulation is a plug configuration, wetting phase is injected uniformly across the left boundary at a rate of $0.11 \mathrm{PV} /$ year and flow is produced on the right boundary at fixed pressure. No flow condition is imposed in top and bottom boundaries. The domain is initially saturated with the non wetting phase. Values for residual saturation are $S_{r, w}=S_{r, n}=0$.

This case is used to validate the capillary pressure implementation. For that purpose the logarithmic capillary pressure model (2.31), with and without permeability dependency, is used:

$$
p_{c}\left(S_{e f f}\right)=-B_{c} \log \left(S_{e f f}\right)
$$

with $S_{\text {eff }}$ as defined in (2.21) and $B_{c}$ : 


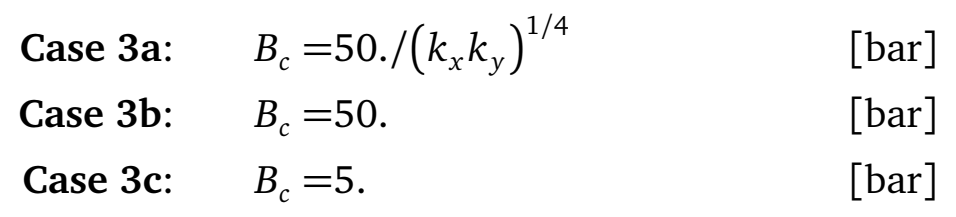

Capillary models with permeability dependency lead to discontinuities in saturation distribution. The model can accurately reproduce this flow feature as can be observed in Figure 4.7 (top right) where wetting phase is accumulated in the interface between different permeability layers.
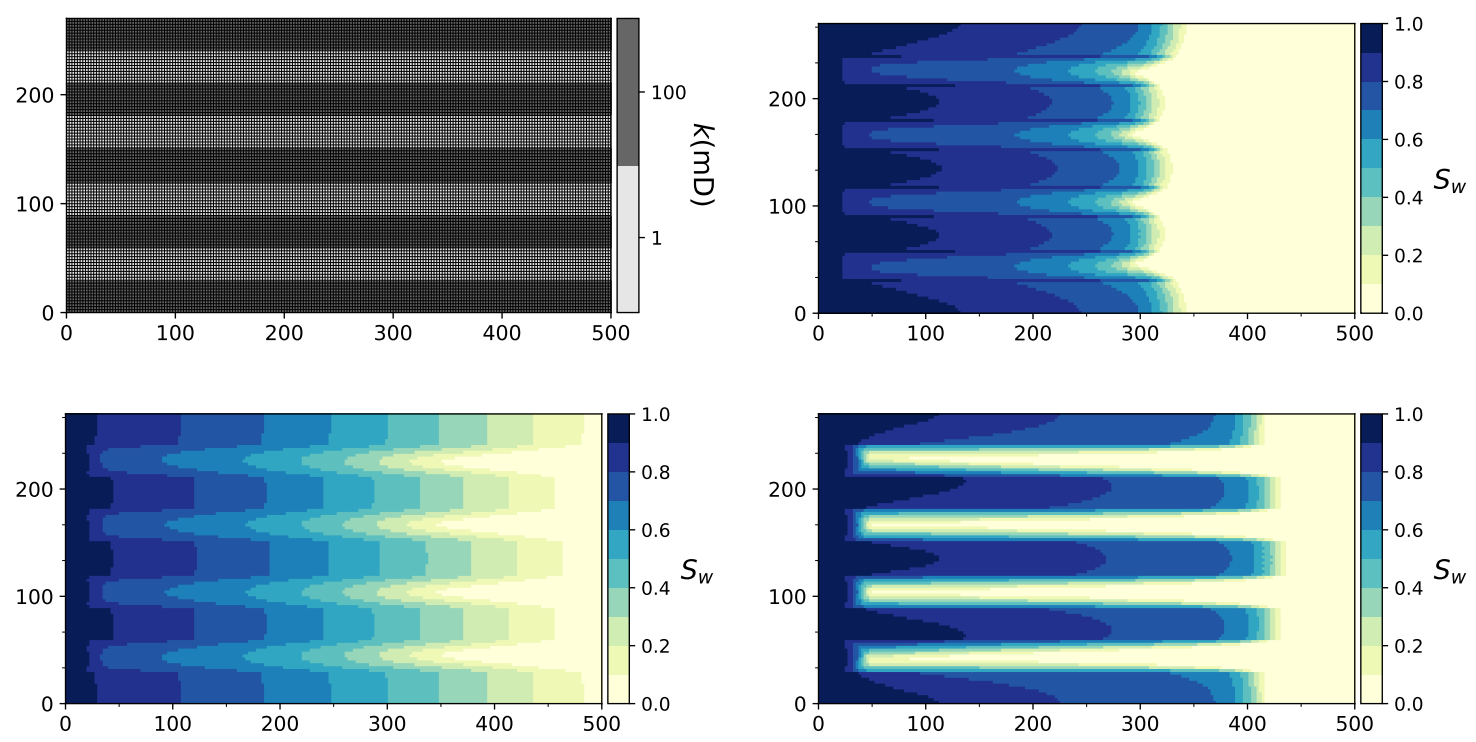

Figure 4.7: Case 3. Top-left: Absolute permeability distribution. Wetting phase saturation distribution after 0.5 PVI for case 3a (top-left), 3b (bottom-left) and 3c (bottomright). Domain size of $500 \times 270 \mathrm{~m}^{2}$ on a $200 \times 100$ mesh.

Results shown in Figure 4.7 are in good agreement with the work of Hoteit and Firoozabadi [2008].

It is important to remark the difference in saturation field with a constant capillary pressure and a permeability dependent model. In Figure 4.7 saturation distributions for three different capillary pressure correlations can be compared.

An error analysis has been carried out for the case 3a with permeability dependent capillary pressure. As the configuration is symmetric, symmetry is expected both in solution and error distribution. Results are shown in Figure 4.8. For tolerance values greater than $10^{-5}$ error accumulated near the inlet leads to unphysical values in saturation but the shape of the distribution and the front location are still accurate. In 

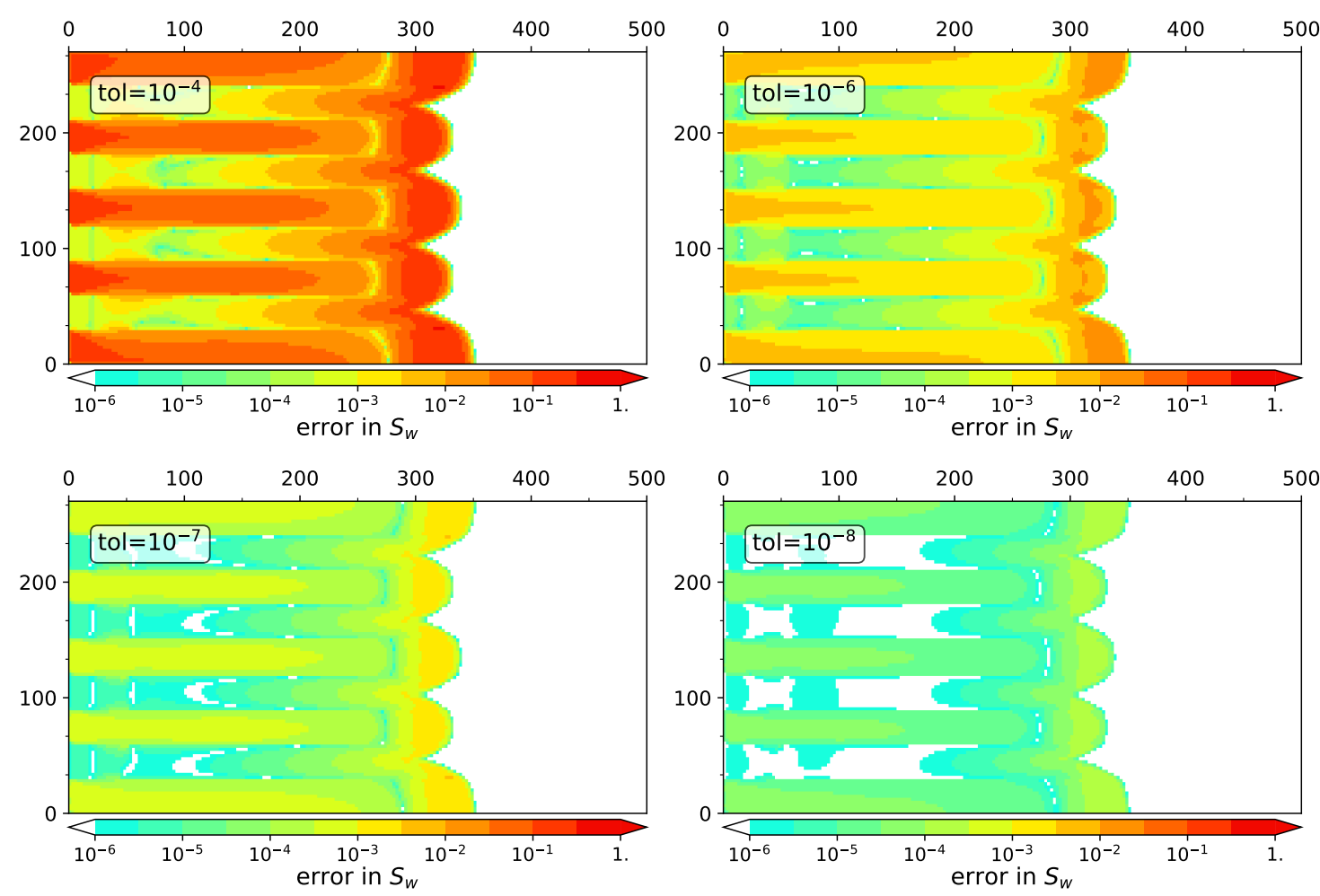

Figure 4.8: Case 3a. Error in wetting phase saturation for different values of solver tolerance at $0.5 \mathrm{PVI}$.

Figure 4.9 the iterative solver efficiency as function of the solver tolerance is plotted. It is shown that for tolerances of $10^{-6}$ computational time can be reduced by a factor of 10 while retaining enough accuracy for most of the applications. Even for low tolerances, iterative solver still outperforms the direct one.

Table 4.4: Computational times for case 3a. $p$ corresponds to the pressure and $S$ to the saturation solver computational time.

\begin{tabular}{rrrr|rrr|rr}
\multicolumn{4}{c}{ IMPES - Exact } & \multicolumn{1}{c}{ IMPES - tol $=10^{-6}$} \\
\hline & time (s) & $p(\mathrm{~s})$ & $S(\mathrm{~s})$ & time $(\mathrm{s})$ & $p(\mathrm{~s})$ & $S(\mathrm{~s})$ & \#Iter & speedup \\
\hline Case 3a & 1075 & 1043 & 21 & 98 & 67 & 21 & 6337 & 11 \\
\hline
\end{tabular}

\subsubsection{Case 4. Heterogeneous plug}

This case is extracted from the work of Yang et al. [2016]. A 2D heterogeneous domain with patches of different permeabilities is considered. Three different configurations 


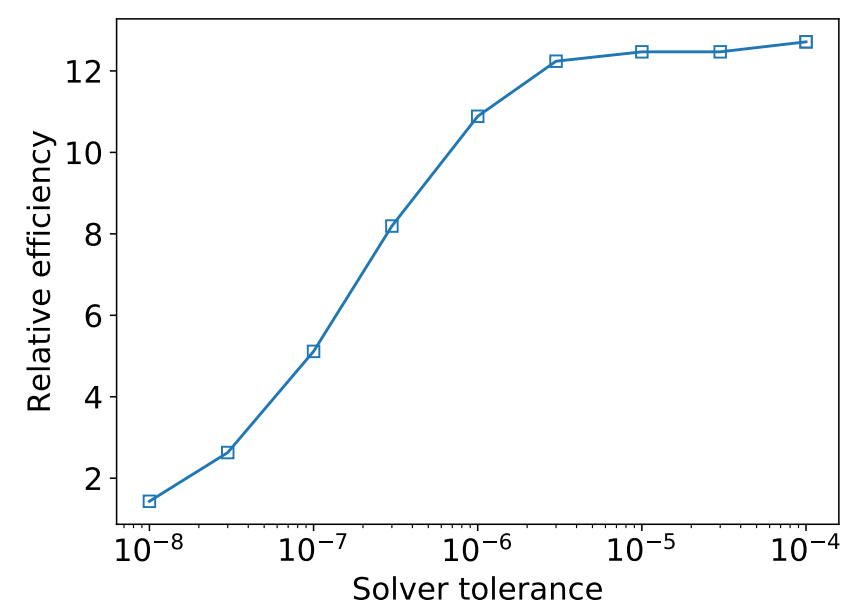

Figure 4.9: Relative efficiency of iterative solver in case 3a with ILU(0) preconditioners.

are tested. Porosity is considered uniform with a value of $\phi=0.2$. In the three configurations, the media is initially fully saturated with non wetting phase and wetting phase is injected uniformly across the left boundary with injection rates described in Table 4.5. Pressure outflow condition is imposed at the right boundary and walls are set at the top and bottom boundaries. In all the cases the permeability is isotropic. The domain in the three configurations has dimensions of 300x150 m and discretised in $100 \times 50$ points.

In case 3 , capillary model has been tested in a domain with quasi $1 \mathrm{D}$ flow, now three permeability configurations with 2D flow will be analysed.

Table 4.5: Parameters for case 4.

\begin{tabular}{lccc}
\hline & Case 4a & Case 4b & Case 4c \\
\hline$n, m$ & 2 & 2 & 2 \\
$S_{r, 1}, S_{r, 2}$ & 0 & 0 & 0 \\
$k_{r, 1}, k_{r, 2}$ & 1. & 1. & 1. \\
$B_{c}\left[\mathrm{bar} \cdot \mathrm{mD}^{1 / 2}\right]$ & 70 & 30 & 100 \\
$\mu_{1}[\mathrm{~Pa} \cdot \mathrm{s}]$ & $0.45 \cdot 10^{-3}$ & $0.25 \cdot 10^{-3}$ & $0.5 \cdot 10^{-3}$ \\
$\mu_{2}[\mathrm{~Pa} \cdot \mathrm{s}]$ & $1 . \cdot 10^{-3}$ & $1 . \cdot 10^{-3}$ & $1.10^{-3}$ \\
Injection rate [PVI/year] & 0.15 & 0.11 & 0.2 \\
\hline
\end{tabular}

In Figure 4.10 the permeability field and the saturation distribution with stream lines of total velocity after 0.5 PVI are shown for the case 4a. Discontinuities in saturation are observed in zones with changes in permeability. Results are in good agreement 
with the work of Yang et al. [2016].
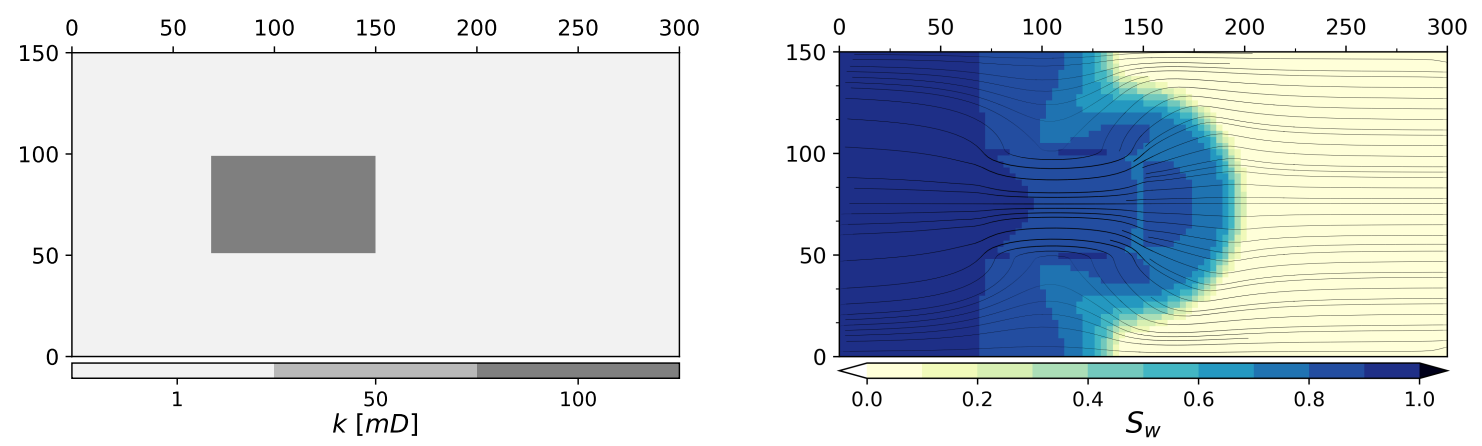

Figure 4.10: Case 4-a. Left: Absolute permeability distribution. Right: Wetting phase distribution after 0.5 PVI with capillary effects. Domain size of $300 \times 150 \mathrm{~m} \times \mathrm{m}$ on a $100 \times 50$ mesh.
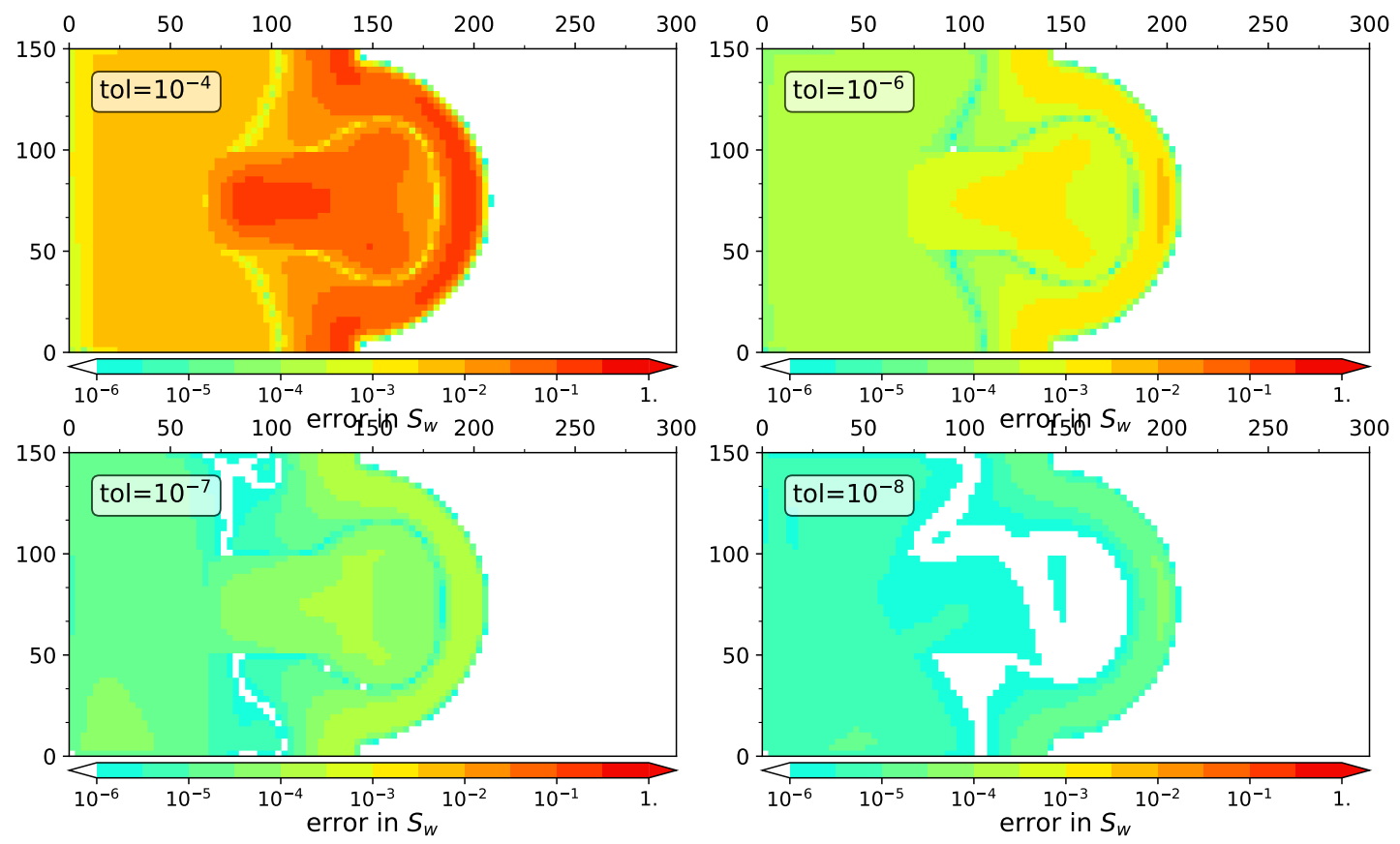

Figure 4.11: Case 4-a. Error in wetting phase saturation for different values of solver tolerance.

In Figure 4.11 the error in saturation distribution referenced to the solution computed with a direct solver is shown for different tolerances. It is important to remark that, despite the large errors for high tolerances the solution is free of oscillations and the overall flow features are well reproduced. Error in saturation fronts is big because 
of the jump effect. Small errors in the position of a front are amplified by the presence of a shock in the saturation distribution.

Case $4 \mathrm{~b}$ has a more complex permeability distribution than case $4 \mathrm{a}$. Saturation after 0.5 PVI is shown in Figure 4.12. The error for different tolerances is represented in 4.13 .
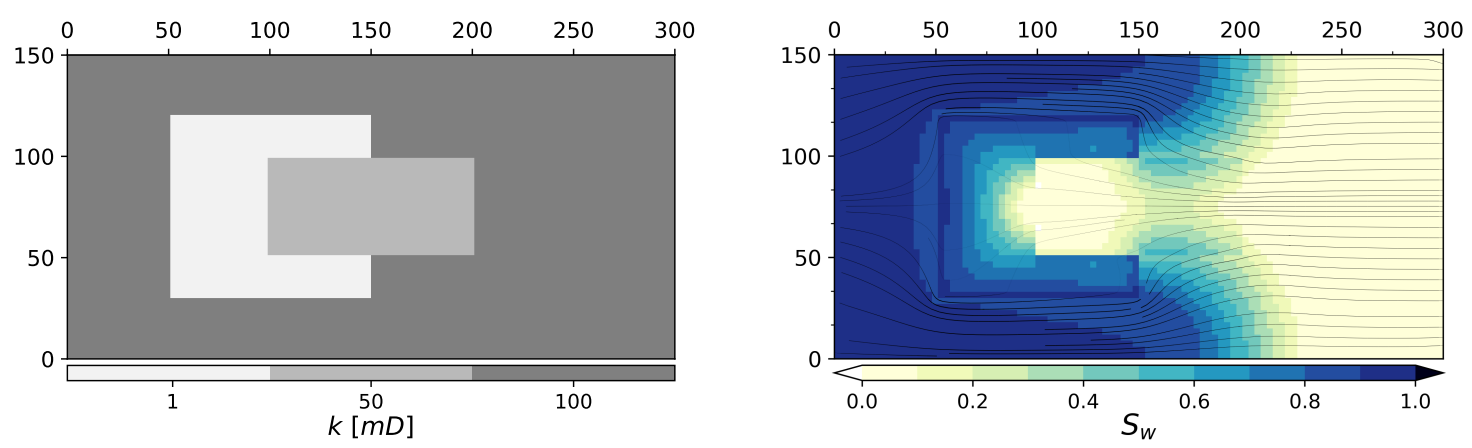

Figure 4.12: Case 4-b. Left: Absolute permeability distribution. Right: Wetting phase distribution after 0.5 PVI with capillary effects. Domain size of $300 \times 150 \mathrm{~m} \times \mathrm{m}$ on a $100 \times 50$ mesh.
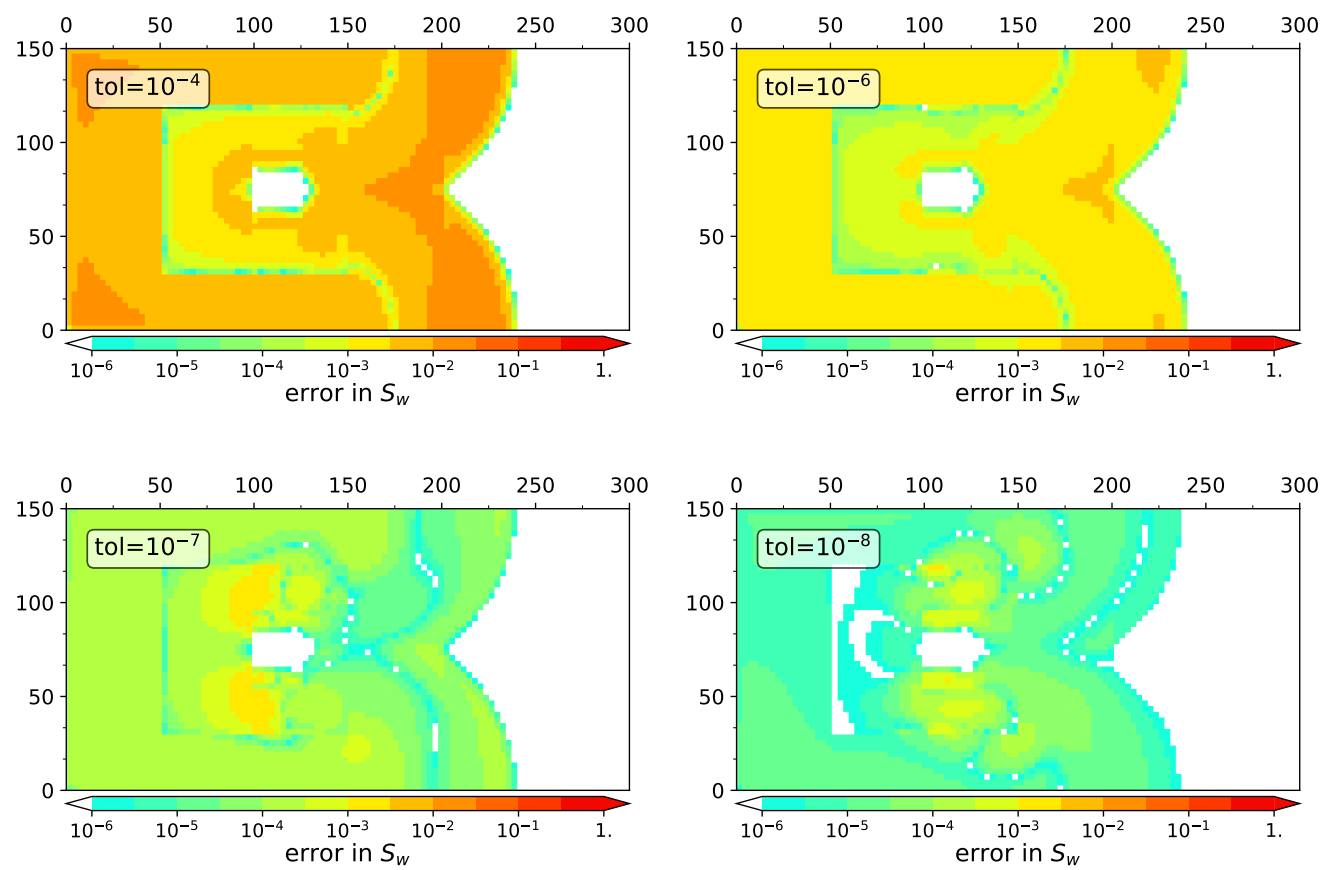

Figure 4.13: Case 4-b. Error in wetting phase saturation for different values of solver tolerance.

In case $4 \mathrm{c}$, Figure 4.14, low permeability obstacles are placed to force $2 \mathrm{D}$ flow. In 
the error plot, Figure 4.15, some oscillations can be observed close to the front at $y=$ 175. These oscillations in the error are associated to small variations in saturation near jumps where saturation is close to the residual saturation. Those small variations are amplified by the logarithmic behaviour of the capillary pressure model that becomes singular for $S_{w}=S_{w, r}$. These oscillations have no apparent effects in the saturation or pressure distributions but can affect the convergence of the iterative solver. This effect can be reduced by limiting maximum capillary pressure value or the minimum $S_{\text {eff }}$ in the relative permeability model (2.31) to avoid unphysical values in capillary pressure.
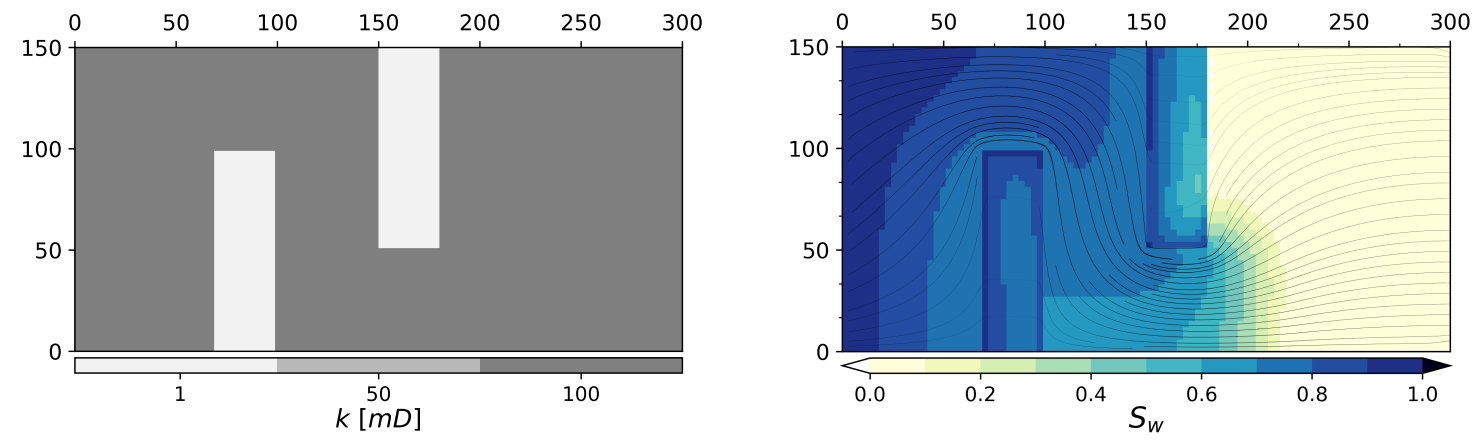

Figure 4.14: Case 4-c. Left: Absolute permeability distribution. Right: Wetting phase distribution after 0.5 PVI with capillary effects. Domain size of $300 \times 150 \mathrm{~m} \times \mathrm{m}$ on a $100 \times 50$ mesh.

Impact of tolerance on the efficiency of the iterative solver is similar to the other analysed scenarios (see Figure 4.16). Iterative solver outperforms the direct one for tolerances above $10^{-8}$ up to a factor of 10 for tolerances above $10^{-5}$. For all the analysed tolerances, including the highest ones, the solution is bounded an reproduce the main features of the flow. The times needed for the pressure and the saturation solver for every case are listed in Table 4.6.

Table 4.6: Computational times and speedup for cases 4a, 4b and 4c, $p$ corresponds to the pressure and $S$ to the saturation solver computational time.

\begin{tabular}{rrrr|rrr|rr}
\multicolumn{8}{c}{ IMPES - Exact } & \multicolumn{1}{c}{ IMPES - tol= $10^{-5}$} \\
\hline & time $(\mathrm{s})$ & $p(\mathrm{~s})$ & $S(\mathrm{~s})$ & time $(\mathrm{s})$ & $p(\mathrm{~s})$ & $S(\mathrm{~s})$ & \#Iter & speedup \\
\hline Case 4a & 102 & 98 & 2.4 & 10.4 & 7.1 & 2.4 & 2747 & 9.8 \\
Case 4b & 143 & 138 & 3.3 & 17.8 & 12.9 & 3.3 & 3861 & 8.0 \\
Case 4c & 228 & 221 & 4.3 & 24.7 & 17.0 & 4.3 & 6143 & 9.2 \\
\hline
\end{tabular}



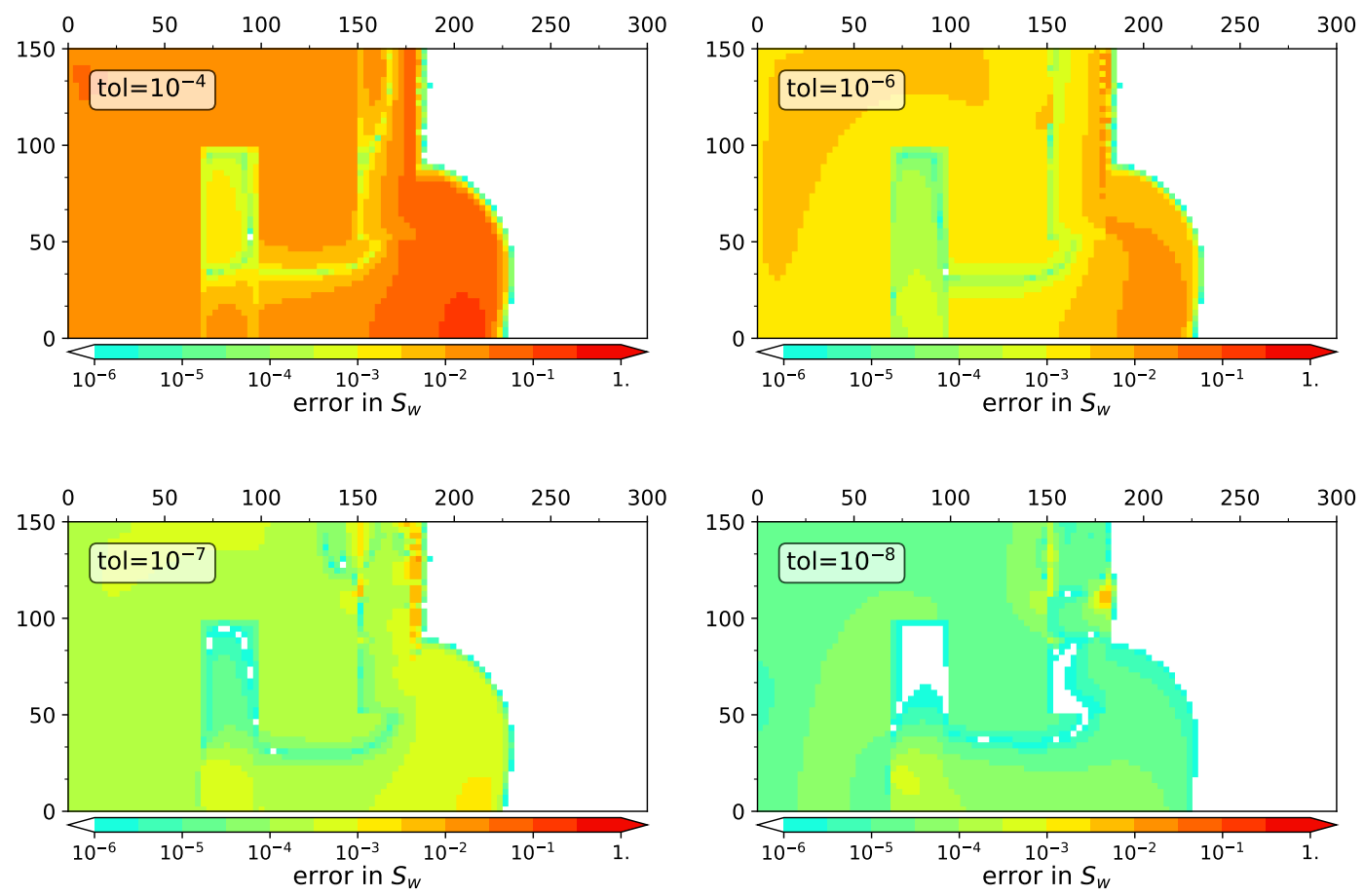

Figure 4.15: Case 4-c. Error in wetting phase saturation for different values of solver tolerance.

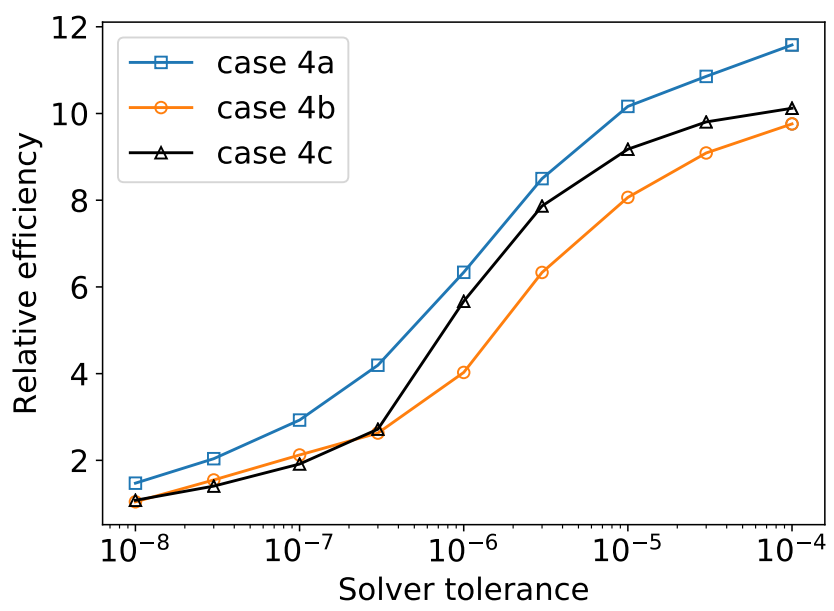

Figure 4.16: Efficiency of iterative solver in case 4 for different tolerances with ILU(0) as preconditioner. 


\subsubsection{Case 5. Five-spot}

This case is a 2D five-spot simulation. This configuration consists on a square domain with four wells placed in the corners of the domain and a fifth well in the middle. Flow is injected in the central well and produced in the other four or vice versa. This configuration is widely used in the oil industry because is the minimum representative configuration required to simulate a matrix of injectors and producers in the secondary oil recovery phase. In this phase gas or water is injected between producer wells to displace the remaining reservoir oil and increase the oil production rate. When a lowviscosity fluid (air or water) displaces a high-viscosity one (oil) and both fluids are immiscible, the interface between the two fluids is unsteady and the fluid with lower viscosity tends to advance in narrow paths in a process called viscous fingering. This process affects drastically the efficiency of the oil displacement. This case will show the ability of the solver to accurately reproduce the viscous fingering phenomenon.

Permeability distribution for this case has been computed with a random porous media generator, using several passes of random noise addition and Gaussian filtering. Wells are surrounded by permeable zones to avoid high unphysical pressures when fixed flow rate is imposed. Water is injected from the four squares of the domain, which is initially saturated with oil. Fluid properties and relative permeability parameters are listed in Table 4.7. Flow is produced from a well in the centre of the domain. Permeability distribution is shown on Figure 4.17. Injection rate is fixed for each injector to $10^{-5} \mathrm{~m}^{2} / \mathrm{s}$. The problem is solved using an uniform grid of $100 \times 100$ points.

Table 4.7: Case 5 parameters.

\begin{tabular}{ccccccccc}
\hline$n$ & $m$ & $S_{r, 1}$ & $S_{r, 2}$ & $k_{r, 1}$ & $k_{r, 2}$ & $\mu_{1}$ [Pa·s] & $\mu_{2}[\mathrm{~Pa} \cdot \mathrm{s}]$ & $P_{c}[\mathrm{bar}]$ \\
\hline 2 & 2 & 0.1 & 0.1 & 1. & 1. & $10^{-2}$ & $10^{-3}$ & 0. \\
\hline
\end{tabular}

In previous test cases the impact of gravity and capillary pressure in the iterative solver efficiency has been presented. In this case the impact of radial flow is analysed. In presence of punctual flow sources, pressure has a logarithmic behaviour close to the injection point and velocities grow with the inverse of the distance to source in this zone. A proper behaviour of the proposed method in this kind of problems is crucial for solving efficiently the near wellbore region.

In the saturation distribution in Figure 4.17 the viscous fingering phenomena can be seen [Sajjadi and Azaiez, 2012], fingers of less viscous phase are formed connecting the injector wells with the producer in the middle of the domain. This phenomena is of high interest for oil industry because of the reduction in the efficiency of oil 

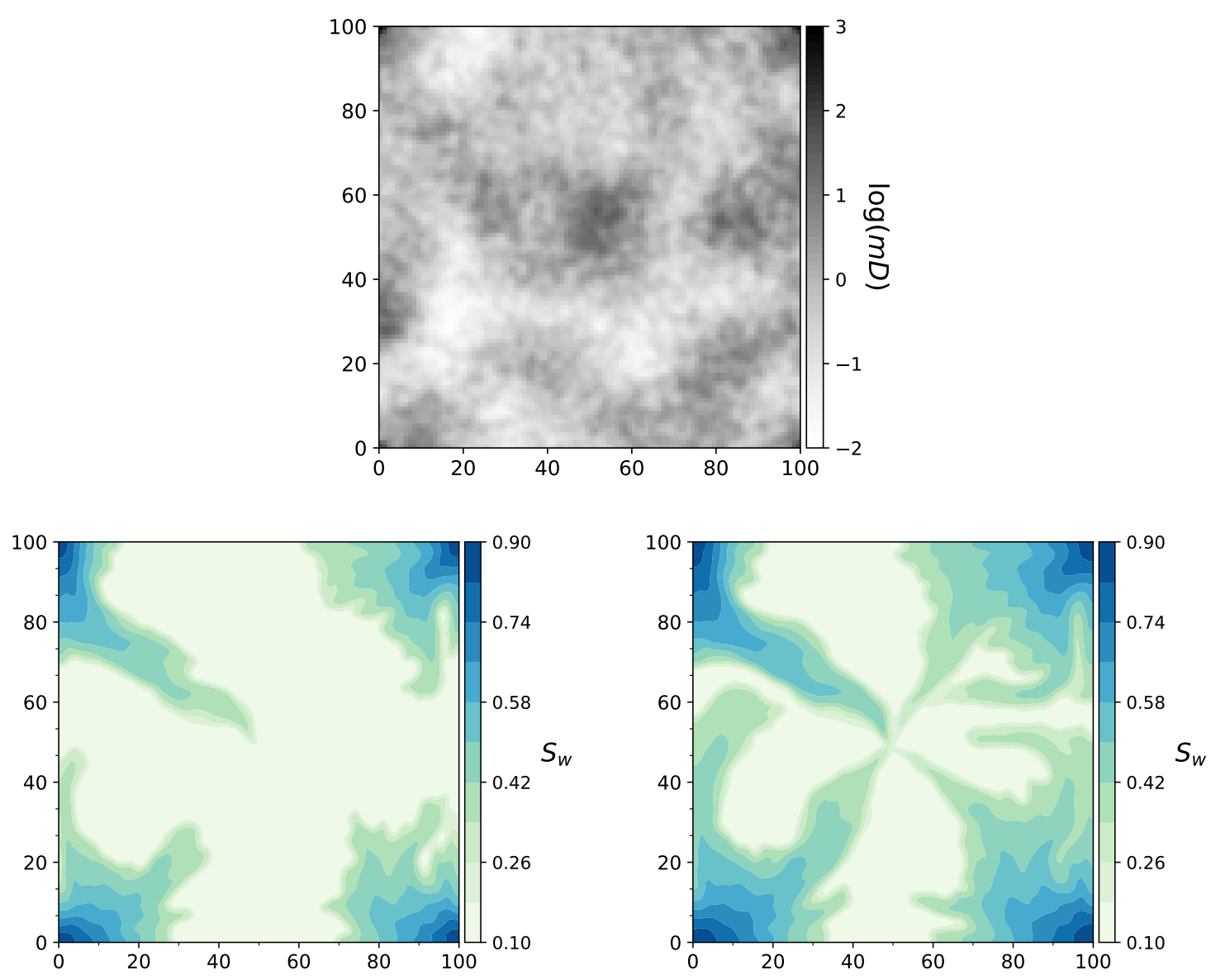

Figure 4.17: Case 5. Top: Absolute permeability distribution. Wetting phase distribution at water breakthrough for the first injector (left) and for the four injector wells (right). Domain size of $100 \times 100 \mathrm{~m} \times \mathrm{m}$ with $100 \times 100 \mathrm{mesh}$.

displacement, thus, an accurate solution of this problems is a key aspect for a reservoir solver.

In the vicinity of wells, pressure distribution varies rapidly and small changes in the saturation field will induce intense modifications in the pressure field in the entire domain. In addition, fingers are unstable and error in pressure field associated with solver tolerance and accumulated error in saturation distribution can lead to a change in the flow configuration. This effect can be noticed in Figure 4.18, for tolerances above $10^{-5}$ the finger flow structure is not well resolved, while for tolerances below $10^{-6}$ the saturation distribution is indistinguishable from the reference solution. The effect of the unresolved finger structure can be observed, in the production plot in Figure 4.19, smoother fronts lead to a more effective oil displacement reflected in a 

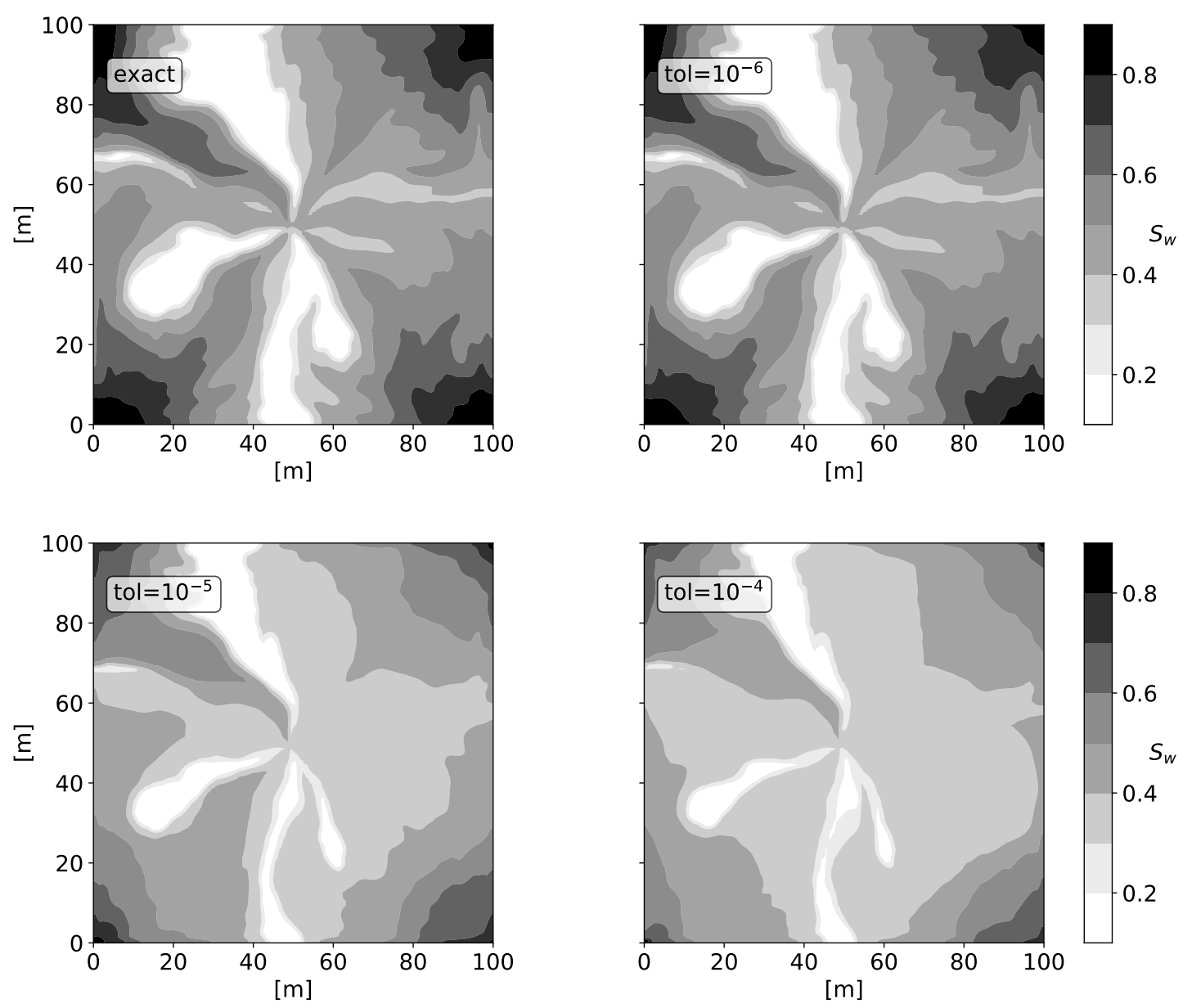

Figure 4.18: Finger structure in saturation distribution in case 5 after 1 year of injection for different solver tolerances. Domain size of $100 \times 100 \mathrm{~m} \times \mathrm{m}$ with $100 \times 100$ mesh. 
lower water cut ${ }^{1}$ value for long simulation times.
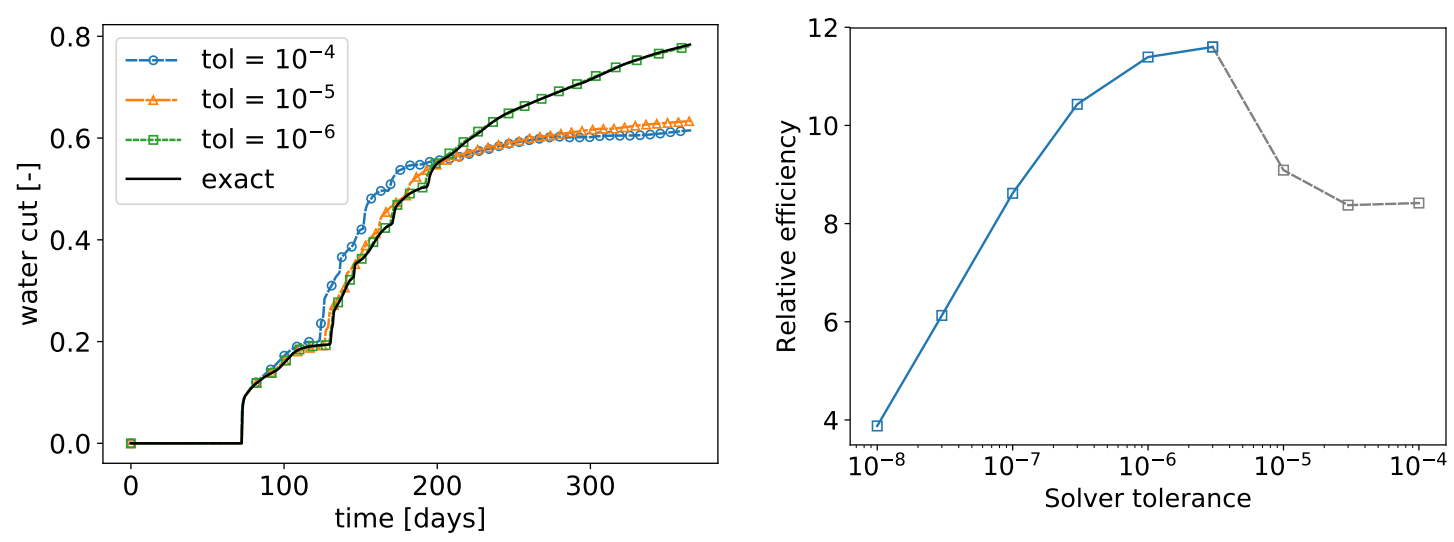

Figure 4.19: Impact of tolerance in case 5. Left: Water cut for different tolerance values. Right: Iterative solver efficiency, grey dashed line show points where the flow configuration is wrongly predicted

In this case, efficiency is higher for low tolerances than in the other analysed cases. The radial configuration of the flow limits the time step, improving the efficiency of the iterative solver due to better initial guesses. The exception is the time close to the breakthroughs, when the water front reaches the vicinity of the wells. In this region the pressure varies rapidly and small changes in mobilities associated to changes in saturation will induce global modifications in the pressure field. In Figure 4.19-right the efficiency plot is shown, and efficiencies greater than 10 have been measured, the details of the computational times are listed in Table 4.8. For tolerances greater than $10^{-5}$ the flow configuration changes, as have been mentioned before, and the efficiency curve is incoherent.

Table 4.8: Computational times and speedup for case 5. $p$ corresponds to the pressure and $S$ to the saturation solver computational time.

\begin{tabular}{rrrr|rrr|rr}
\multicolumn{4}{c}{ IMPES - Exact } & \multicolumn{1}{c}{ IMPES - tol $=10^{-6}$} \\
\hline & time $(\mathrm{s})$ & $p(\mathrm{~s})$ & $S(\mathrm{~s})$ & time $(\mathrm{s})$ & $p(\mathrm{~s})$ & $S(\mathrm{~s})$ & \#Iter & speedup \\
\hline Case 5 & 4425 & 4281 & 91 & 393 & 251 & 91 & 57900 & 11.3 \\
\hline
\end{tabular}

\footnotetext{
${ }^{1}$ Water cut: The ratio of water produced compared to the volume of total liquids produced
} 


\subsubsection{Case 6. SPE10 dataset 2}

This simulation has been performed with the 3D version of the simulator, developed for Repsol during this thesis. Some quantitative results are shown to prove the ability of the methodology to deal with long run simulation on industrial size problems. The permeability distribution of the reservoir is shown in Figure 4.20, fast variations in the permeability field up to 8 orders of magnitude are present. In Figure 4.21 the distribution of porosity is shown. This tricky configuration, including null porosity regions and sharp permeability variations are well resolved by the numerical algorithm showing no oscillations or unphysical variable magnitudes.

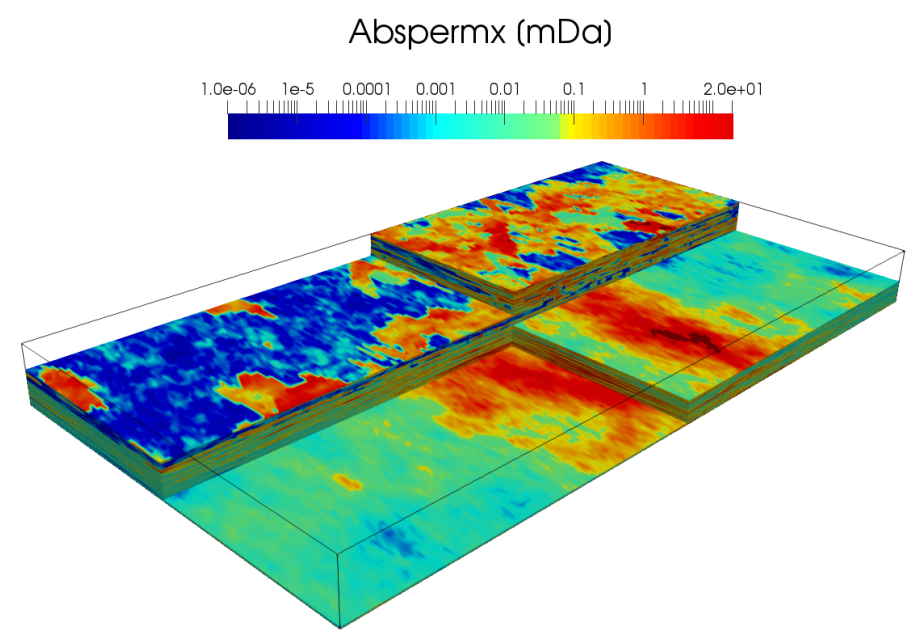

Figure 4.20: Permeability distribution for the SPE 10 dataset 2. Cuts showing different domain layers.

The simulation setup consists on fives wells. One injector in the center of the domain in which water in injected at a rate of $5000 \mathrm{bbl} /$ day and four producers with a fixed pressure of 4000 psi ( 275.8 bar). The domain is saturated with oil with irreducible water saturation. Fluid properties are listed in Table 4.9. Rock and fluid compressibility, as well as the dependence of the viscosity with pressure are neglected in this simulation.

Table 4.9: Case 6 fluid properties.

\begin{tabular}{ccccccccc}
\hline$n$ & $m$ & $S_{r, 1}$ & $S_{r, 2}$ & $k_{r, 1}$ & $k_{r, 2}$ & $\mu_{1}[\mathrm{~Pa} \cdot \mathrm{s}]$ & $\mu_{2}[\mathrm{~Pa} \cdot \mathrm{s}]$ & $\boldsymbol{g}\left[\mathrm{m} / \mathrm{s}^{2}\right]$ \\
\hline 2 & 2 & 0.2 & 0.2 & 1. & 1. & $3 \cdot 10^{-3}$ & $0.3 \cdot 10^{-3}$ & 0 \\
\hline
\end{tabular}

One thousand days of injection have been simulated. The pressure distribution and the position of the water front after 35 days of simulation is shown in Figure 4.22. 


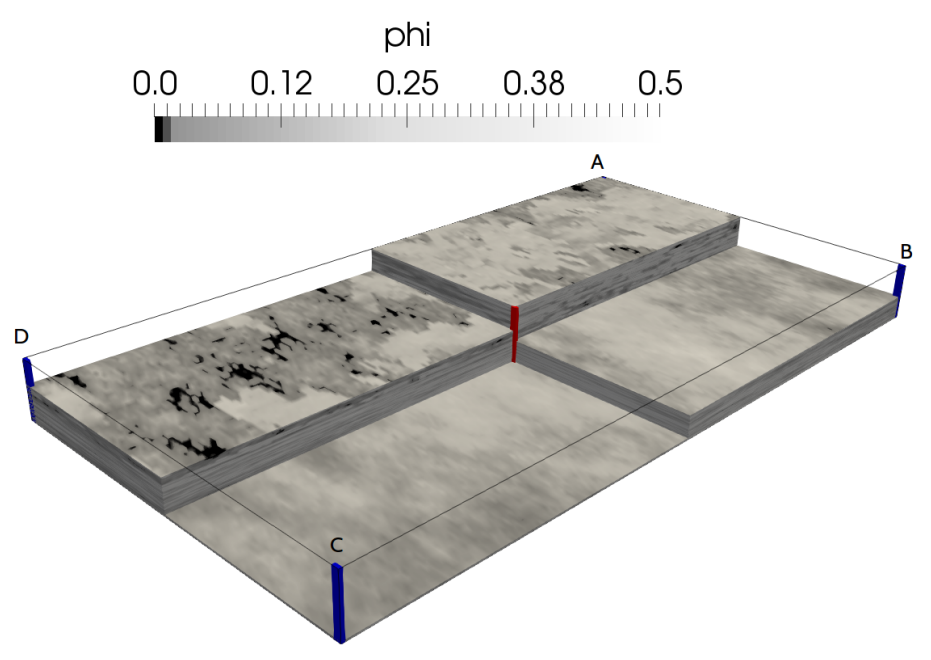

Figure 4.21: Porosity distribution for the SPE 10 dataset 2. Cuts showing different domain layers. The locations of the injector (red) and producers wells (blue) are marked in the figure.

The water cut history for the four producers is plotted in Figure 4.23. No oscillation or unphysical variable magnitude have been observed. Results are in good agreement with the published results [Christie and Blunt, 2001] despite of the simplifications in the simulation mentioned before.

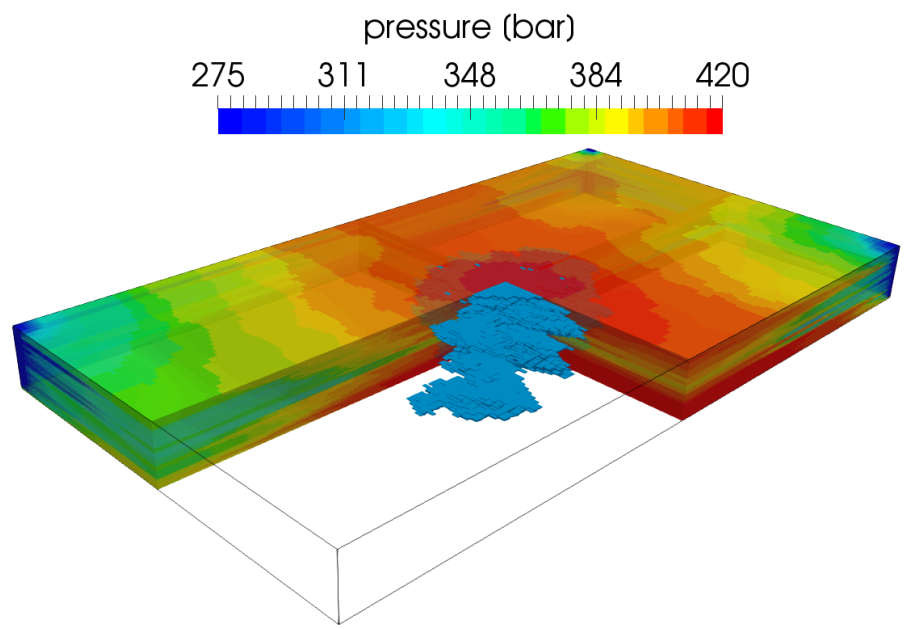

Figure 4.22: SPE10 dataset 2. Pressure distribution and water front position after 35 days of simulation. 


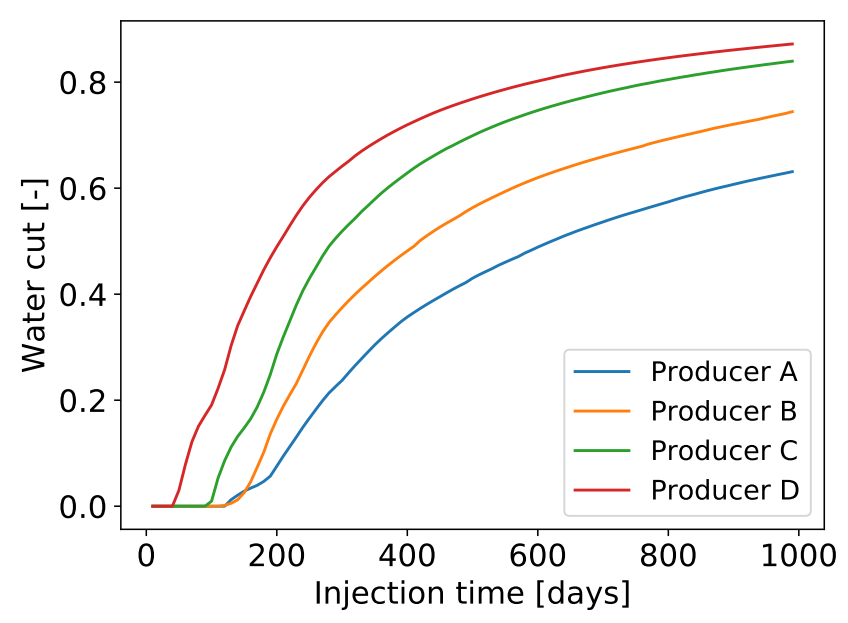

Figure 4.23: SPE10 dataset 2. Water cut history for the four producers.

\subsection{Analysis of iterative solver}

\subsubsection{Impact of preconditioner}

The success of iterative linear solvers depends highly on the preconditioner used. Depending on the problem, some preconditioners will outperform others but there is, generally, no best one. For this solver, 3 preconditioners have been analysed. The monitor variable of convergence is the divergence of total velocity per time step, as defined in (3.38). This variable, used as error monitor, is almost problem independent as shown in Section 4.1.

In Figure 4.24 the convergence history for the GMRES is shown. For a fixed time step the pressure equation has been solved using a fixed number of GMRES iterations for the three different preconditioners analysed. In all the cases, the pressure distribution from the previous time step has been used as initial guess. On the one hand, the left plot shows that from the point of view of the number of iterations, the most expensive the preconditioner, the fastest the convergence. On the other hand, when comparing the total solution time, the cost of computing and applying the preconditioner comes into play. Now for higher tolerances, cheaper preconditioners present lower solution times while for lower tolerances the expensive preconditioners perform the best.

It should be noticed (Figure 4.24-right) that the three methods outperform the direct one for the case analysed, where only one order of magnitude is needed to converge from the initial guess to get the desired divergence tolerance. This behaviour is similar in the other test cases as can be drawn from the efficiency plots for the 

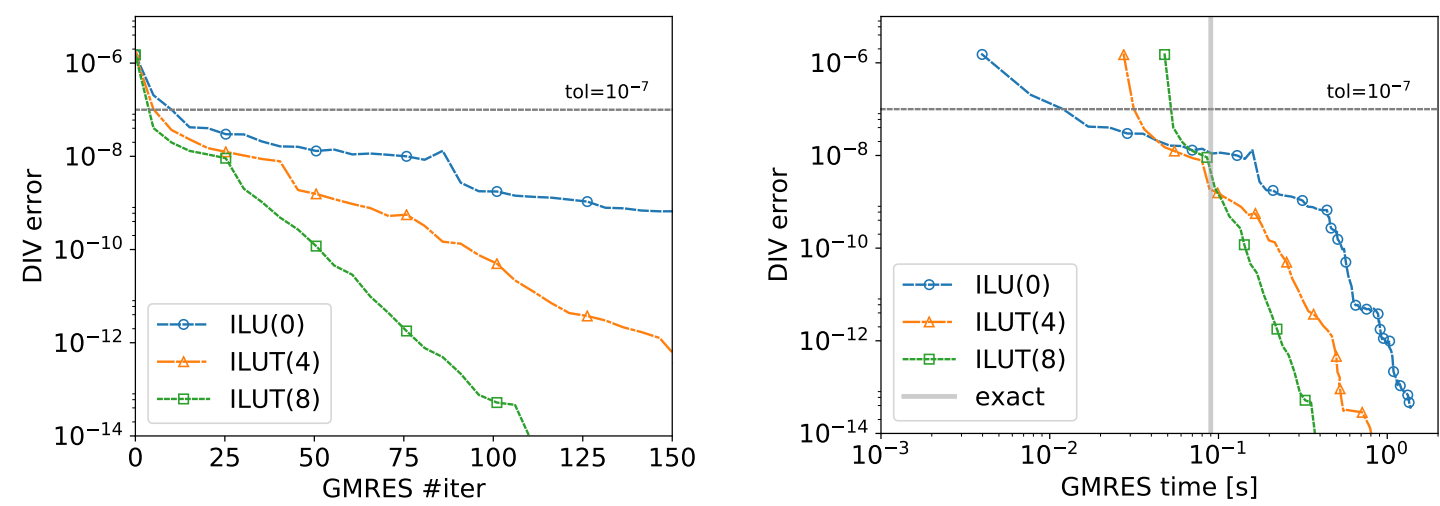

Figure 4.24: Convergence history for the GMRES linear solver for three different preconditioners. On $y$-axis, the error in total velocity divergence associated with the GMRES solution and on $x$-axis number of GMRES iteration (left) and GMRES solution time (right). Markers every 25 iterations. Analysis corresponds to case 3 after 0.2 PVI with a tolerance of $10^{-7}$.

Case 2

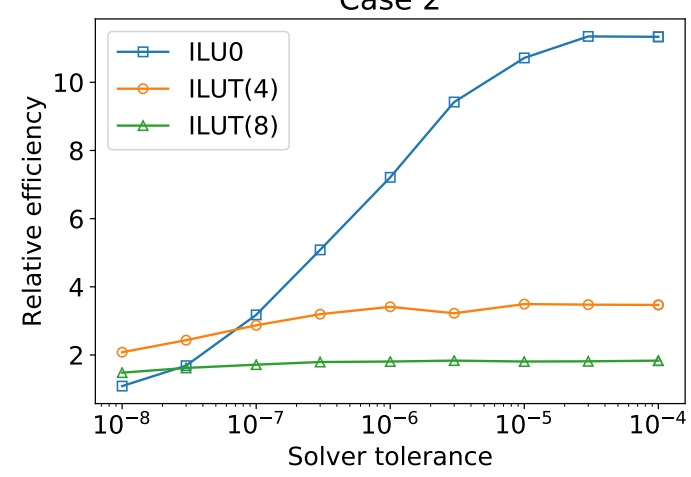

Case 3

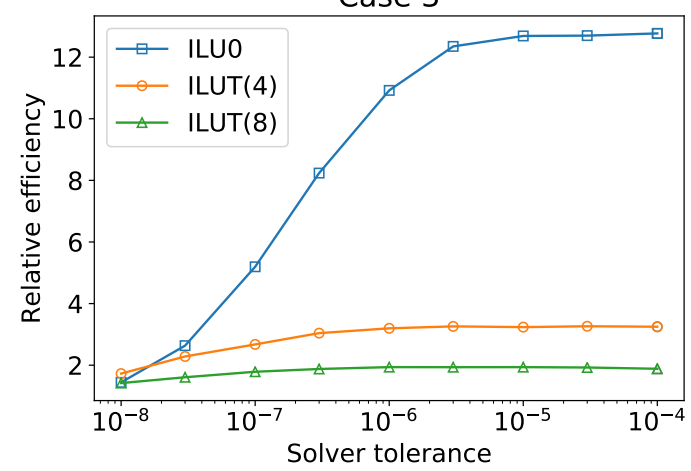

Figure 4.25: Efficiency of iterative solver for different preconditioners function of the divergence tolerance.

different preconditioners shown in Figure 4.25

Both GMRES-ILUT(8) and GMRES-ILUT(4) present an approximately constant cost with respect to the Solver tolerance, as most of the computational time is spent in the computation of the preconditioner, i.e., the effect of the number of iterations of the GMRES is small. On the contrary, GMRES-ILU(0) presents a highly variable computational cost as time is not spent in the computation of the preconditioner (it is cheap) and therefore the relative effect of the number of iterations of the GMRES is bigger 


\subsubsection{GMRES restart}

GMRES algorithm becomes impractical when the Krylov subspace size, $m$, grows. For that reason GMRES can be restarted after $m$ iterations using the last approximation as initial guess. This strategy accelerates the operations required during the GMRES iteration but can stagnate convergence if the system matrix is ill conditioned and not positive definite, which is normally the case for the system arising from the pressure equation. The stagnation can be mitigated using a good preconditioner.
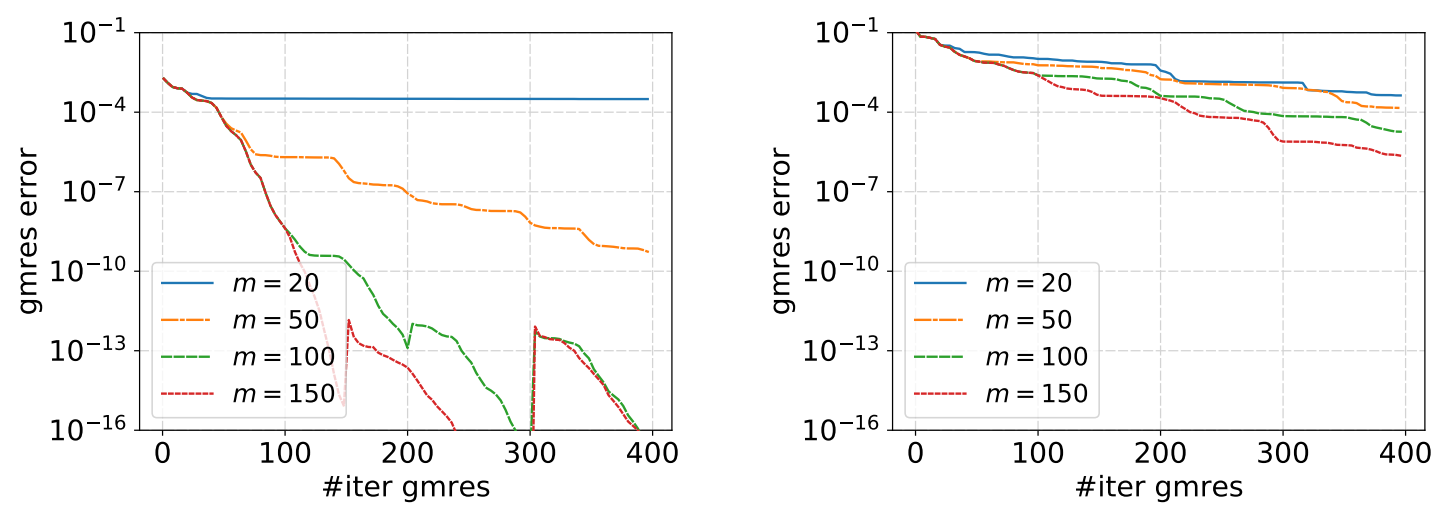

Figure 4.26: Impact of the restart iteration $m$ in the convergence of the GMRES for case $4 \mathrm{a}$ (left) and case(5) using ILU(0) preconditioner.
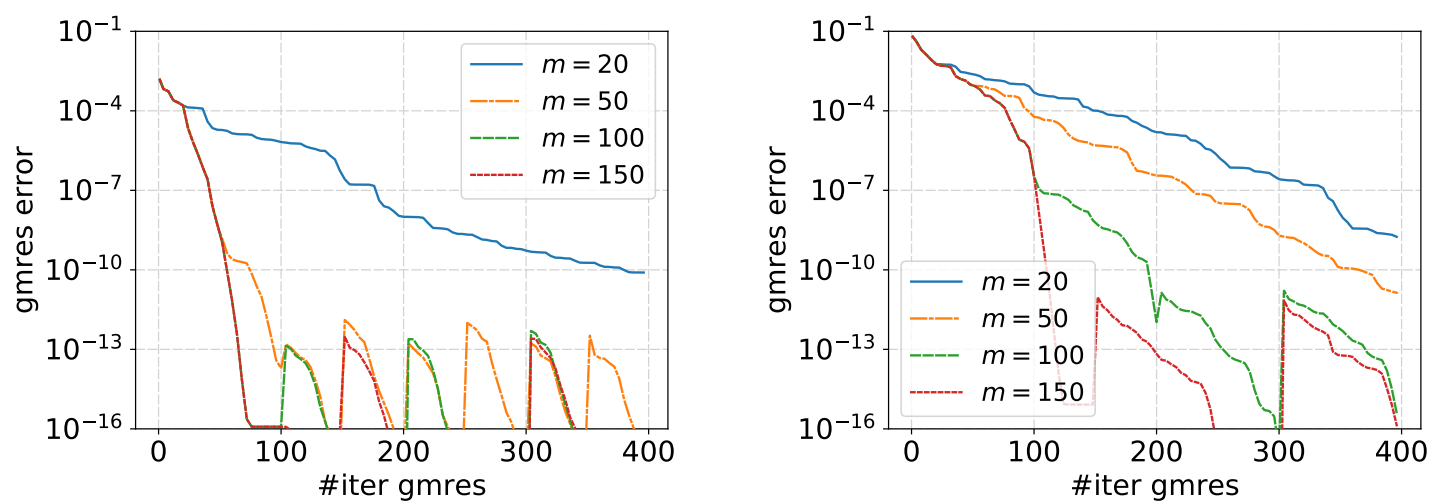

Figure 4.27: Impact of the restart iteration $m$ in the convergence of the GMRES for case 4a (left) and case(5) using ILUT(4) preconditioner.

Some test cases have been carried out to analyse the impact of restart in the convergence of GMRES. From the results shown in Figures 4.26 and 4.27, typical restart 
values below 50 can easily decelerate the convergence or even stagnate it when ILU(0) or ILUT are used, for this reason a value of 150 has been chosen for all test cases in this work. 


\section{chapter 5}

\section{MULTIPHASE 2D IMPES SOLVER}

The solver introduced in this chapter, simulates 2D, 2-phases, immiscible, non reactive flow through porous media. The $2 \mathrm{D}$ code presented here, is an academic version of the industrial version developed for the company Repsol during this thesis. This code is implemented for validation and optimization of the physical models, algorithms and numerical methods used in the industrial version. In the following sections, the implementation of the methodology described in previous chapters is detailed. The solver is programmed in Fortran 2008 and uses the VTK format for input/output.

The media is defined using a rock file where porosity, permeabilities and wells are described. Boundary conditions, fluid properties, relative permeability as well as other simulation parameters are supplied using a control file.

Mesh is provided within the rock file. The solver uses a rectangular cartesian grid with uniform or variable spacing. The type of the boundary conditions is given with the simulation kind defined in the control file. Three different configurations are available: pressure plug, flux plug and wells.

Pressure plug In this simulation pressure and saturation are imposed in the inflow (left boundary), pressure in the outflow (right boundary) and no flow condition is imposed in the top and bottom boundaries.

Flux plug In this simulation constant flow for each phase is imposed in the inflow (left boundary) and pressure in the outflow(right boundary). No flow condition is imposed in the top and bottom boundaries.

Wells In this simulation symmetry/no-flow condition is imposed in the four boundaries. An arbitrary number of wells can be defined in the rock file. Wells are described 
in the configuration file and can be defined as pressure wells, where pressure is fixed or flux wells, where a fixed flux for each phase is given.

\subsection{Input/Output files}

The format for the input output data files is VTR due to compatibility issues. This format is used natively by the visualization software Paraview and is part of the VTK (Visualization Tool Kit project). A thorough search revealed no available libraries for input/output VTR format in Fortran and those have been developed for this thesis.

\subsubsection{File format}

VTR format is based on XML standard. Files are ASCII plain text and store the data associated to the variables as well as the grid information. The format and the library support cell-centred and vertex-centered data of kinds double and single precision floats and integers. An example of a rock file is listed below. This file includes the grid description ( $x$ and $y$ distributions) and the values of the absolute permeability tensor (AbspermX and AbspermY), porosity and markers. The marker identifies the point as porous domain (marker $=0$ ) or well (marker $>0$ ). The rock described in the example file is shown on Figure 5.1.

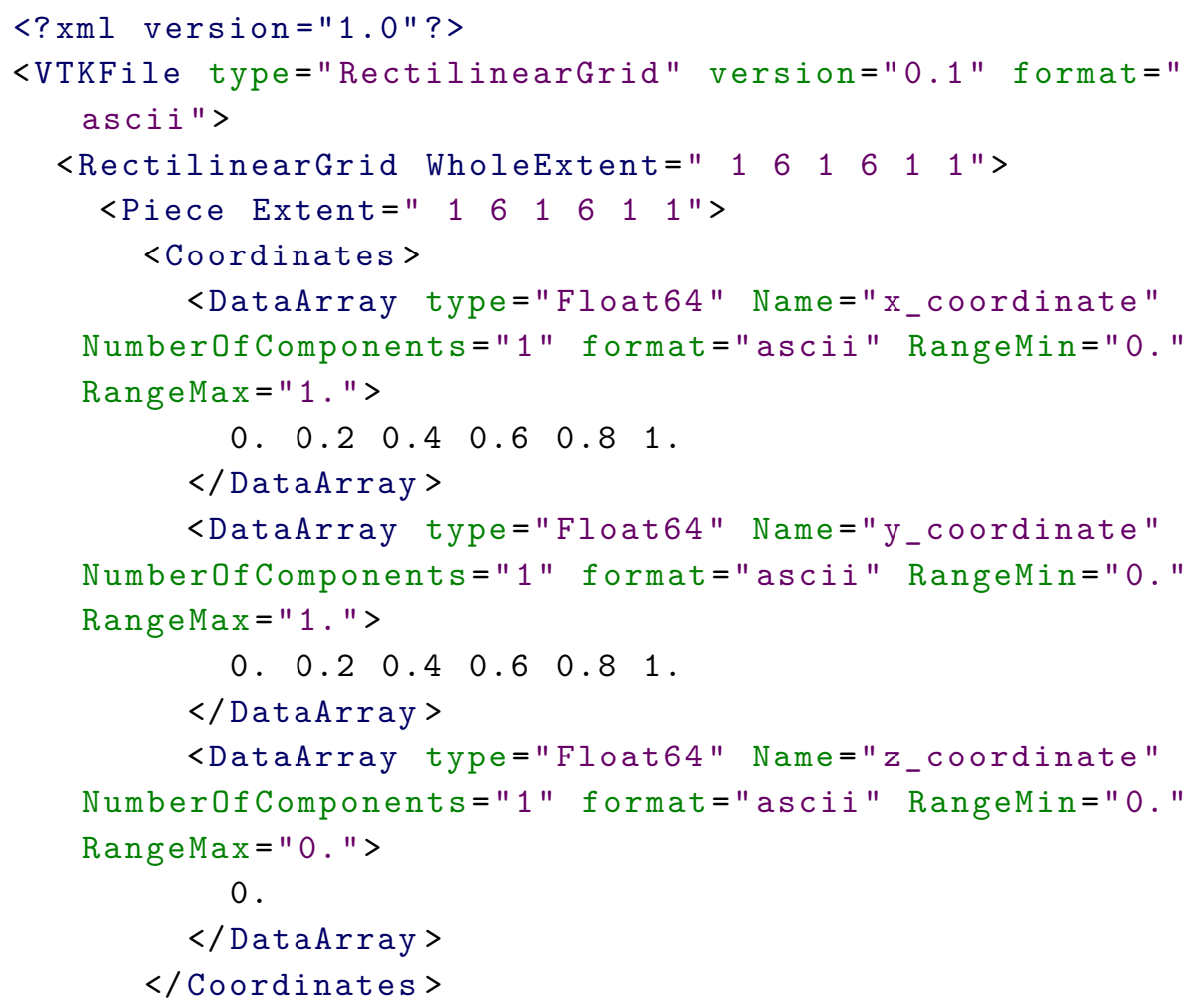




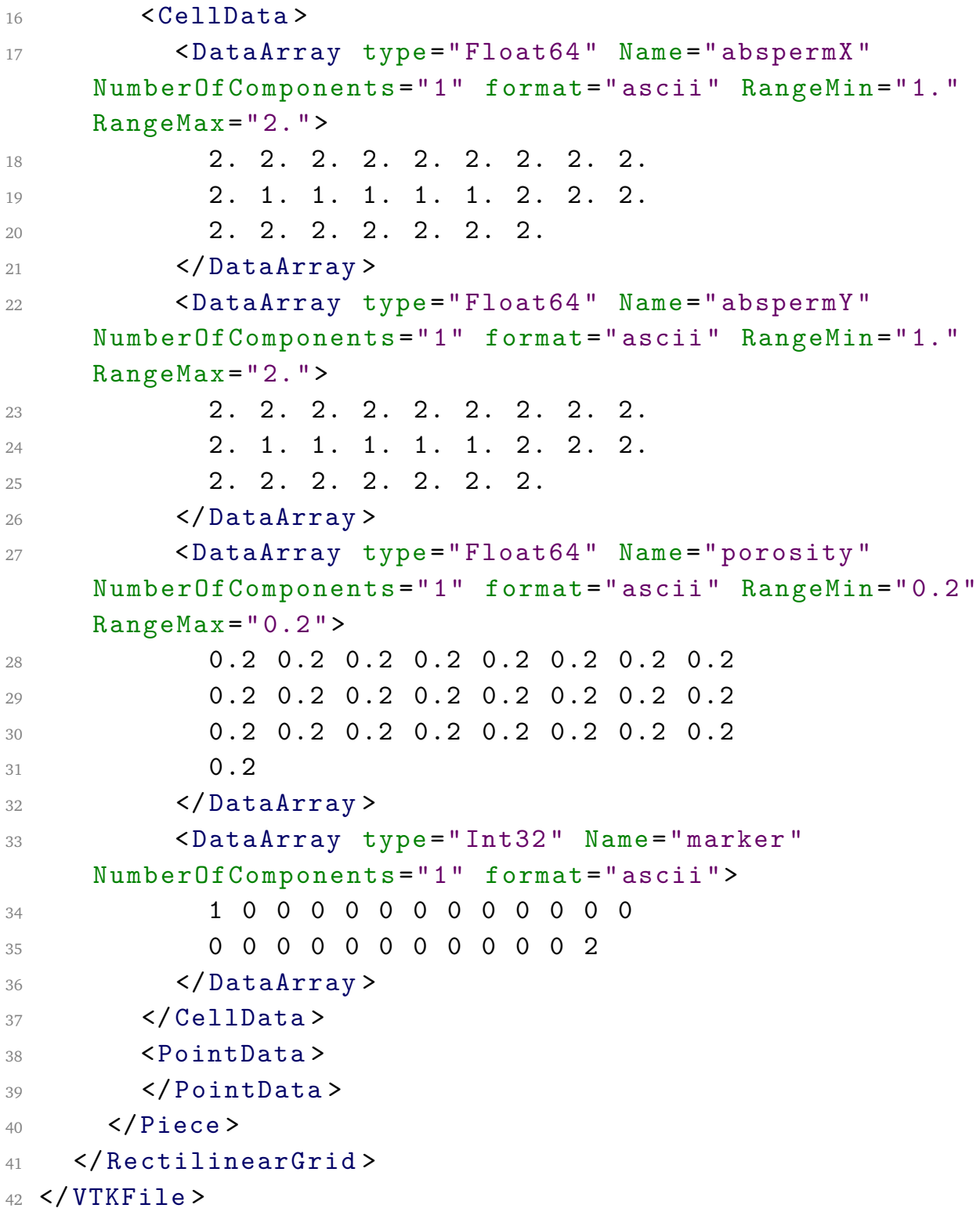

Listing 5.1: Rock file example

\subsection{Implementation methodology}

One of the goals of the simulation code is to serve as a base for further development, for that reason, the code has been designed using a modular structure implemented with modern object-oriented Fortran 2008. The sketch of the code is shown in Figure 5.2. A brief explanation of the main blocks of the code is given below. 


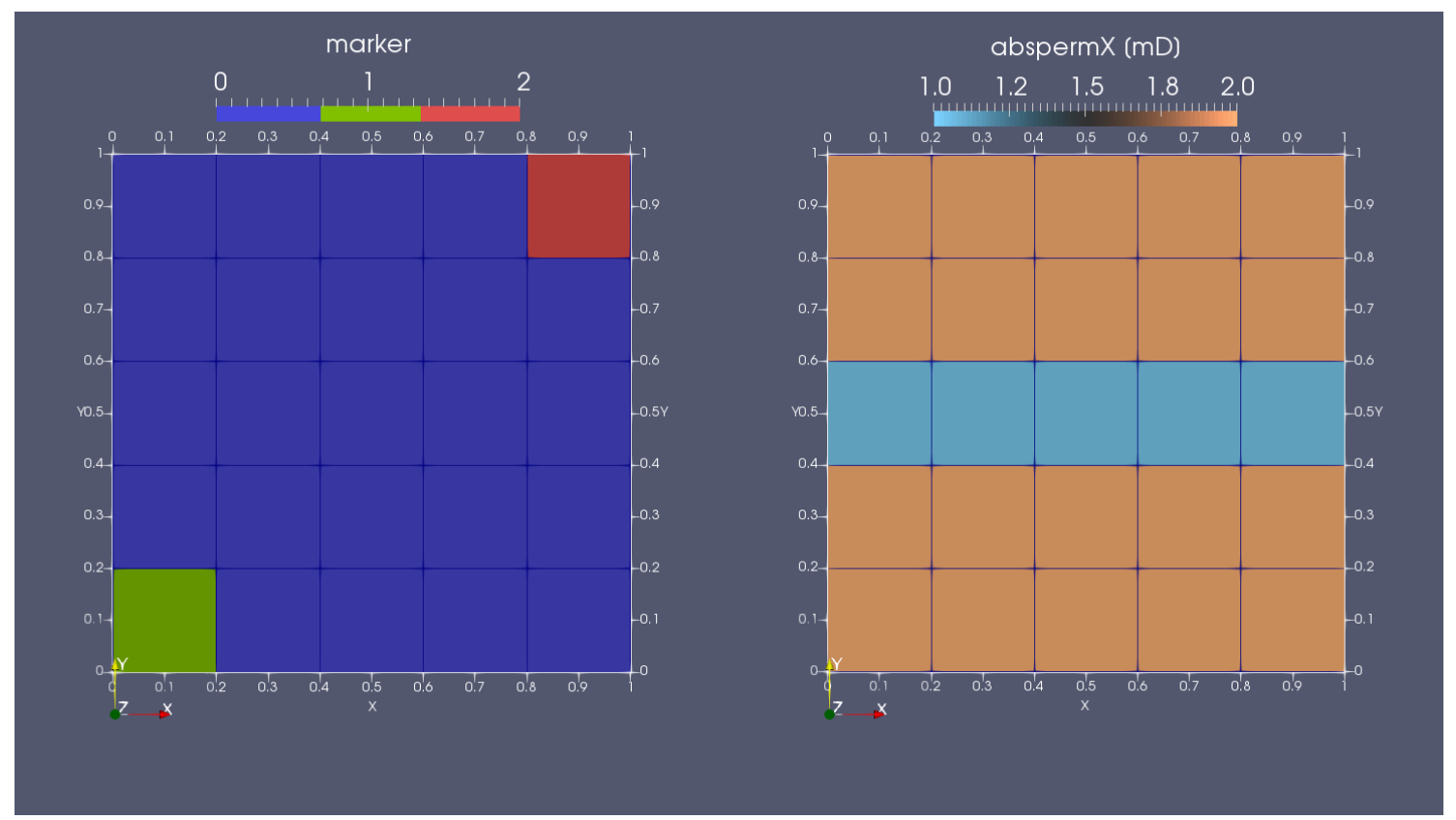

Figure 5.1: Rock file example visualized with Paraview.

Simulation module This is the main block, where the objects instances of the classes described below are created.

Config class This class contains all the configuration parameters and the method for reading it from the parameter file.

Data class This class contains all the data structures required by the solver. Data is organised using structure-of-arrays paradigm (see Appendix A). Each variable is stored in a 2D array. The class includes a method for reading the rock file which provides rock data as well as the grid and problem dimensions. Point data, such as pressure or saturation; face data, like mobilities or fluxes; control data, such as time steps or tolerances and monitoring variables are defined in this class.

IMPES solver Here, the temporal integration is implemented. Each time step, successive calls to the pressure solver, saturation solver and mobilities update are made according to the IMPES algorithm.

Mobilities In this module the procedures for updating the relative permeabilities and computing the face mobilities are implemented, these procedures are invoked by the IMPES solver. 


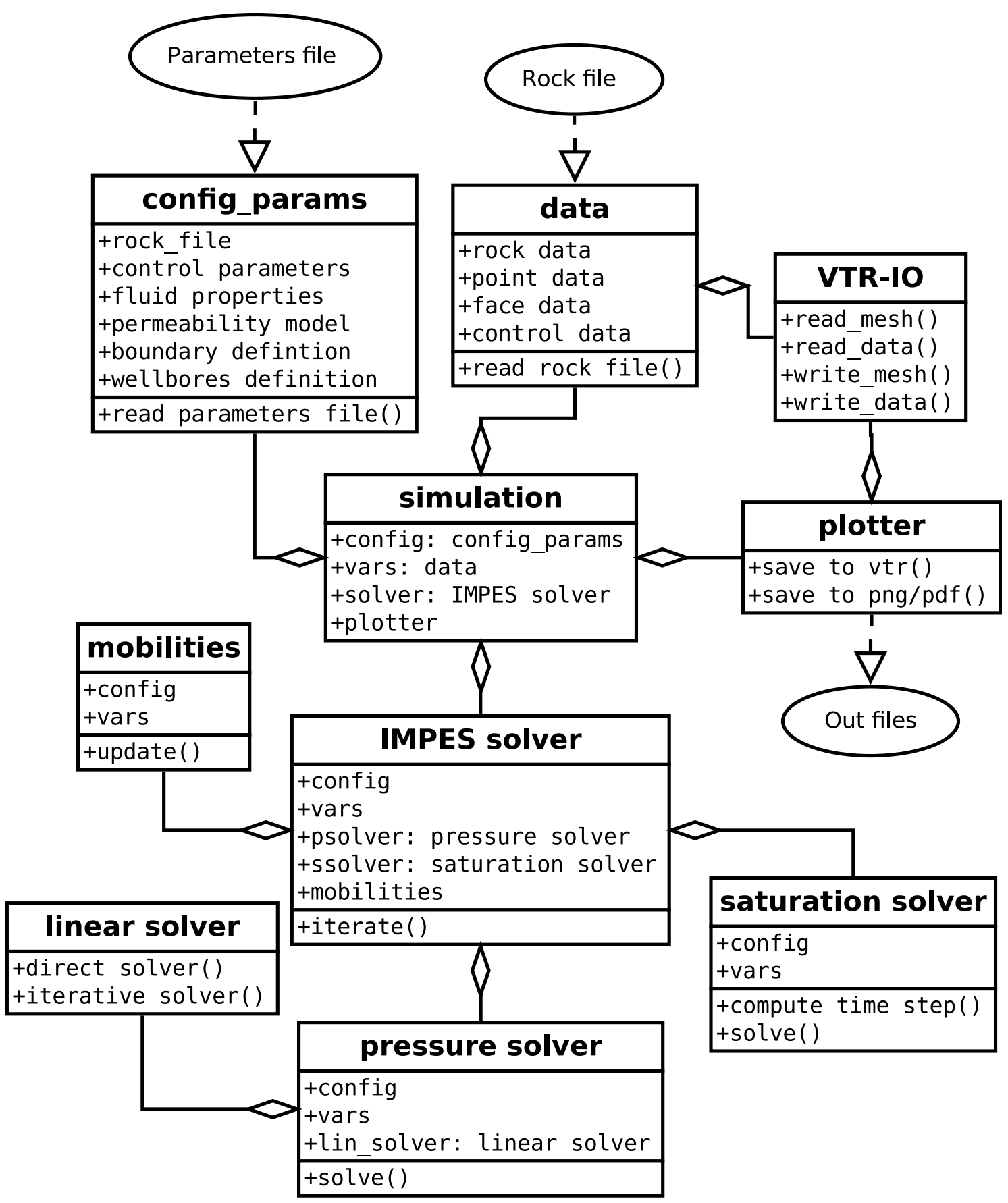

Figure 5.2: Simplified diagram of the Darcy2D code. 
Saturation solver This class contains the routines that compute face fluxes and time step required for updating the saturation field.

Pressure solver This class includes the code for computing the system matrix derived form the discretisation of the pressure equation and the appropriate boundary conditions. On each call, the matrix is assembled and the linear system is solved invoking the linear solver.

Linear solver This class acts as a wrapper for the MKL routines for solving sparse linear systems [Intel Corporation, 2017]. A direct solver based on PARDISO [Amestoy et al., 2001] and a iterative solver based on RCI-FGMRES with several preconditioner options are implemented.

VTR-IO This module contains the routines needed for reading and writing files in VTR format.

Plotter In this module the routines that generate the ouput of the code are defined. Results can be produced in VTR format or directly into pdf/png or live-plot through the included python-matplolib wrapper.

\subsubsection{Sparse matrix storage}

From the discretisation of the pressure equation (2.32), the matrix of the linear system has a pure penta-diagonal structure, associated to the 2D 5-point finite differences scheme. The matrix is extremely sparse for typical sized problems, with only five non zero elements per row. In this scenario the use of sparse storage and algebra is required to be able to handle industrial scale simulations. For example, using dense algebra a matrix associated to a problem defined on a mesh of size $200 \times 200$ would require $40000 \times 40000$ elements $\times 8$ Byte per elemet $=12.8 \mathrm{~GB}$ of RAM, only for the matrix storage. In the same case, using sparse algebra will require, for a naive non compressed storage, $40000 \times 5$ elements $\times 8$ Byte per elemet $+40000 \times 2$ indices $\times$ 4 Byte per index $=1.92 \mathrm{MB}$, roughly ten thousandth of the memory needed in the dense case.

There are some strategies for storing sparse matrices in a compact (compressed) form. In this code, one of the most used formats, the CSR (Compressed Sparse Row) ${ }^{1}$ is used. This format is supported by the majority of sparse linear algebra libraries (e.g. MKL [Intel Corporation, 2017], , MUMPS [Amestoy et al., 2001], PETSc [Balay et al., 2016], etc).

\footnotetext{
${ }^{1}$ This format is sometimes referred as Compressed Row Storage (CRS)
} 
Compressed Sparse Storage (CSR) This format is valid for every non zero elements distributions since there is no assumption about the sparsity pattern and does not stores any unnecessary elements.

A matrix $M$ of size $m \times n$ and $N N Z$ non zero values is defined using three vectors(A,IA,JA) where:

- A is a vector of length $N N Z$ that contains all the non zero the values of $\boldsymbol{M}$ sorted by rows (row-major order).

- IA is of length $m+1$ and is defined recursively, $\operatorname{IA}(1)=1$ and $\operatorname{IA}(i)=\operatorname{IA}(i-1)+$ number of non zero elements in the $i-1$ row.

- JA is of length $N N Z$ and contains the column indices of each non zero element in the matrix $M$.

The values of the row $i$ in $M$, stored in A, can be accessed using the vector IA in the following way: $\mathrm{A}(\operatorname{IA}(i): \operatorname{IA}(i+1)-1)$ and to the corresponding columns indices: $\operatorname{JA}(\operatorname{IA}(i): \operatorname{IA}(i+1)-1)$, thus, vector IA is often referred as row pointer or row ptr. An example of the format is given below:

$$
\begin{aligned}
M & =\left(\begin{array}{lllll}
1 & 0 & 0 & 0 & 0 \\
2 & 1 & 1 & 0 & 0 \\
0 & 0 & 1 & 3 & 0 \\
0 & 0 & 0 & 0 & 4
\end{array}\right) \\
\mathrm{A} & =(1,2,1,1,1,3,4) \\
\mathrm{IA} & =(1,2,5,7,8) \\
\mathrm{JA} & =(1,1,2,3,3,4,5)
\end{aligned}
$$

\subsubsection{Parallelisation}

The implementation of the solver described in previous sections uses two different parallelisation strategies. On the one hand loops over points or faces are parallelised using OpenMP as well as the maximum or minimum of an array that uses reductions under OpenMP. On the other hand, the linear solver uses the multithread version of MKL-Pardiso, for the direct solver, and the multithreaded version of the FGMRES, for the iterative one, both included in the MKL Library. 
Explicit loops Most of the computational cost of the explicit saturation solver is related to loops over all the points or faces in the domain with no interdependencies. This kind of problems, known as embarrassingly parallel will present a high parallelization efficiency. To improve the efficiency of the implementation, nested loops due to the iteration over $x$ and $y$ dimensions can be collapsed to work as one bigger loop, reducing the overheads associated to the threads creation/destruction. A typical OpenMP point-loop is listed below:

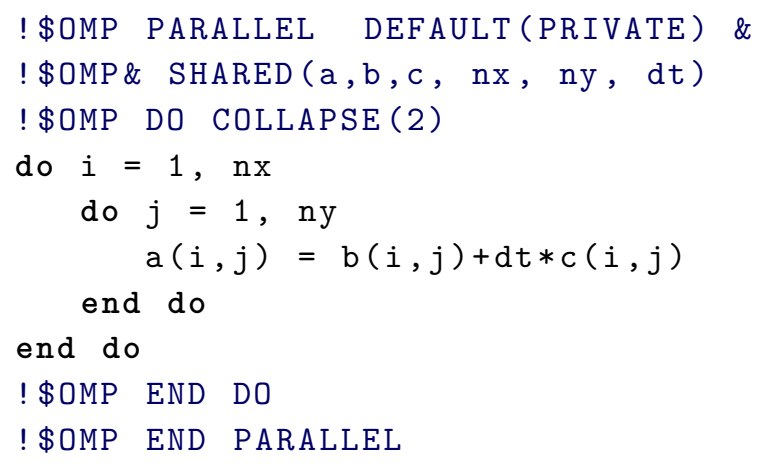

Listing 5.2: OpenMP nested loop example

Reductions Operations such as sum, min, max or average, are known as reduction operations, taking as input an array of values an retuning a single scalar. OpenMP can lead with this kind of operations using the REDUCTION clause. The syntax used in the code for a min and a max reduction is listed below:

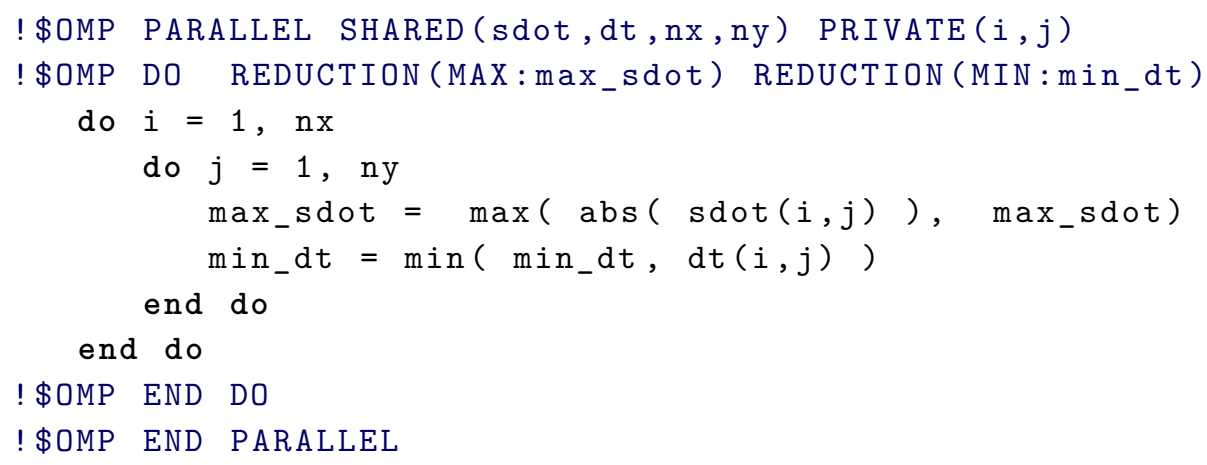

Listing 5.3: OpenMp reduction example

Linear solver The parallelisation of this part of the code relies on the multi-thread version of MKL. Pardiso [Schenk and Gärtner, 2004], the direct solver uses a parallelisation at operation level (multithread vector operations) and at algorithm level, obtaining a modest efficiency with the sizes involved in the analysed cases. FGMRES uses only multithread vector operations but the algorithms are serial. Multithread 
vector operations are only efficient when the overheads associated to the thread creation/destruction are negligible compared to the cost of the serial operation which only occurs with huge arrays. With the sizes of the problems proposed in this work, the parallel performance of the iterative linear solver is poor.

Results of scalability are shown in Figure 5.3. As can be observed, the parallel performance of the mobilities computation, based on explicit loops, is nearly ideal. The speedup of the saturation solver which includes explicit loops, reductions an serial operations drops as the number of CPUs increases. In addition, loops in saturation solver are very light, with operations involving only a couple of additions or multiplications. This kind of loops are typically memory bounded, where speed is limited by memory bandwidth instead of by CPU speed, taking almost no advantage of parallelisation in shared memory architectures. This effect is studied in Appendix A.
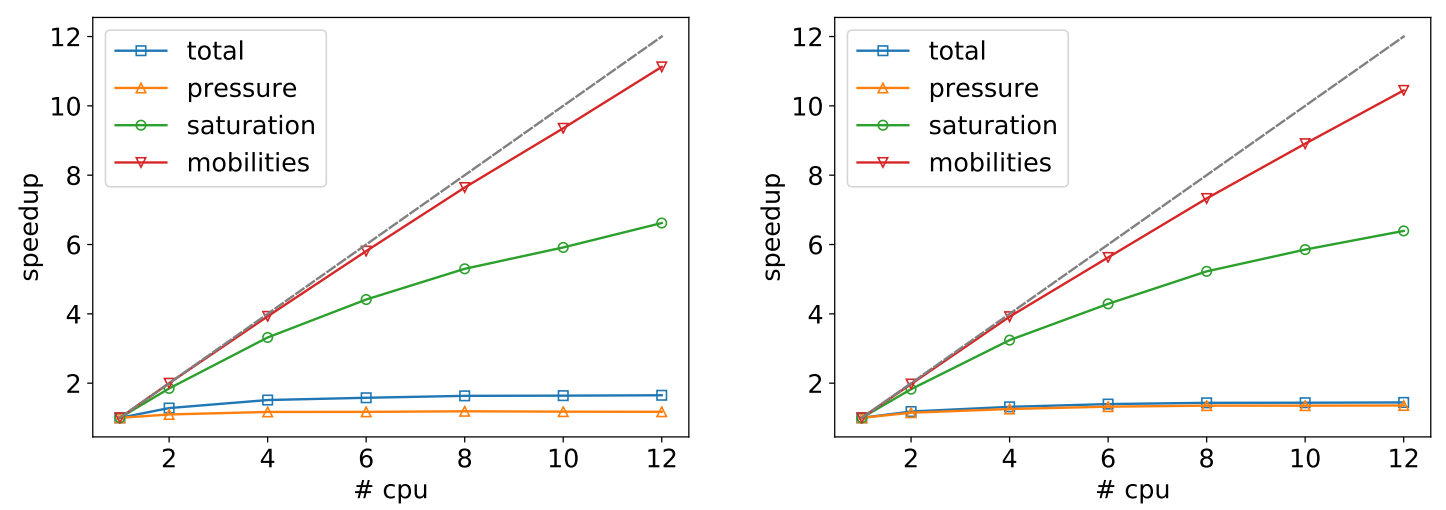

Figure 5.3: Scalability for case 3 with tol $=10^{-7}$ (left) and direct solver (right). 



\section{CONCLUSIONS}

An improved methodology to simulate the multiphase immiscible flow through porous media in the near wellbore region has been developed.

The method is very efficient to solve problems where temporal accuracy is required. The developed numerical scheme takes advantage of the time step restriction of the IMPES method to reduce the computational cost of the implicit pressure solver by using an iterative linear solver. Furthermore, the relation between the iterative linear solver tolerance and the accuracy of the solution has been studied. The validity of the developed strategy to improve the performance of the method without compromising the accuracy of the solution has been shown for complex problems including wells, gravity and capillary effects.

An improvement in computational time of up to an order of magnitude with respect to the conventional IMPES has been measured in a wide range of simulations. This reduction is similar to the one obtained with fully implicit methods for some configurations, but with higher temporal accuracy and less memory requirements. These features can be relevant in some applications of high interest such as detailed near wellbore simulations or transient processes.

The GMRES based solver has been tested in a wide range of scenarios to prove the robustness of the mathematical and numerical models. One of the main drawbacks when dealing with iterative solvers is the unknown efficiency and the problem dependency. Through a wide variety of tests, it has been proven that, despite showing variable performance, the algorithm is robust and outperforms other reported strategies.

In addition, this thesis has been used to apply modern object-oriented Fortran to an industrial code. The methodologies, as well as modules and libraries developed are already been used in others ongoing projects.

The 2D Darcy solver presented in this work is the initial step of a new line in the research group in the field of multiphase flow in porous media. The solver is the base for further development including compressibility, reactive flows and compositional models. 

Appendices 



\section{appendix $A$}

\section{DATA STRUCTURE. AOS VS SOA}

One of the first decisions to make when coding a CFD algorithm is the chose of the data structure. Usually, this kind of codes use a certain number of variables associated with the discretisation points and/or the discretisation faces. There are, mainly, two criteria in these cases: defining an structure that stores all the data associated with a point or a face and declare an array of this kind (Array of Structures, AOS), or defining an array for each variable (Structure of Arrays, SOA). Apart from the coding syntax, the main difference between these two approaches is the memory layout of the data. In the first case, all the data associated with a point is contiguous in memory and in the second case, it is the data associated to a variable the one that is contiguous. Traditionally, when programming for CPU, memory bandwidth has not been an issue, but modern processors with tens of cores and vectorization capabilities have increased drastically the computation performance and the memory throughput can become a bottleneck. If memory bandwidth is the limiting factor, the data structure becomes determinant in the overall code performance.

In serial codes, memory bandwidth is not the limiting factor due to the size and hierarchy of cache memory in modern processors but, when using multi-thread parallelisation and vectorization, the computational capability increases and thus the requirement of memory throughput.

For measuring the impact of the data structure in the code performance the program listed below has been used. The code consist on a variable update typically found in a 2D finite differences algorithm, coded for a AOS and a SOA structures. Every single update operation has a very low arithmetical cost (only addition and multiplications). The number of point updates per second is computed as a measure of the efficiency. The program is compiled with Intel Fortran Compiler 2017 with the following optimization flags:

ifort -03 -xavx -parallel -qopt-report=2 
Tests have been carried out on a workstation with dual Intel(R) Xeon(R) CPU E5-2630 @ $2.30 \mathrm{GHz}$ (with Turbo Boost and Hyperthreading disabled) and 32 GB RAM DDR3 @ 1333MHz.

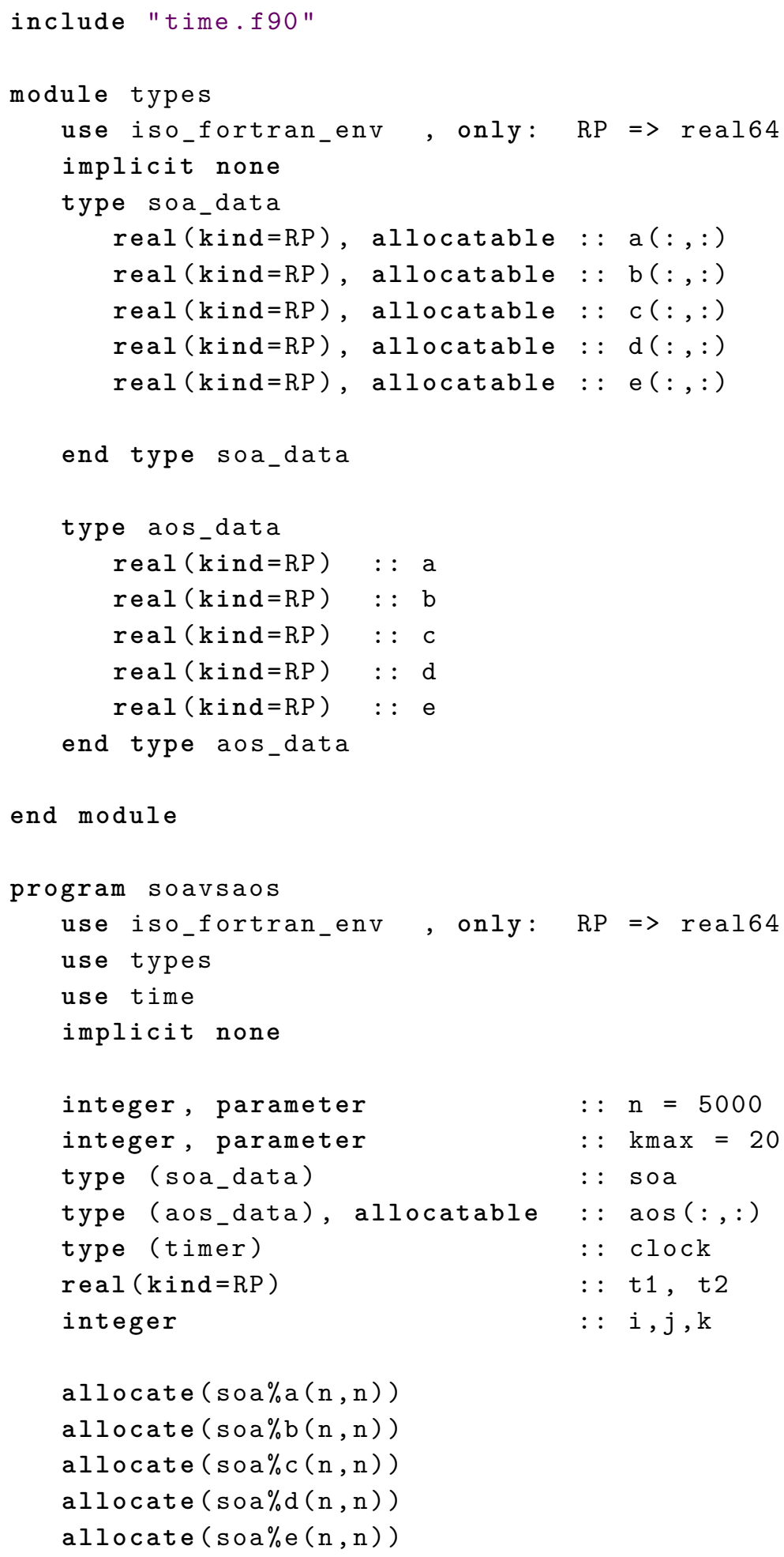




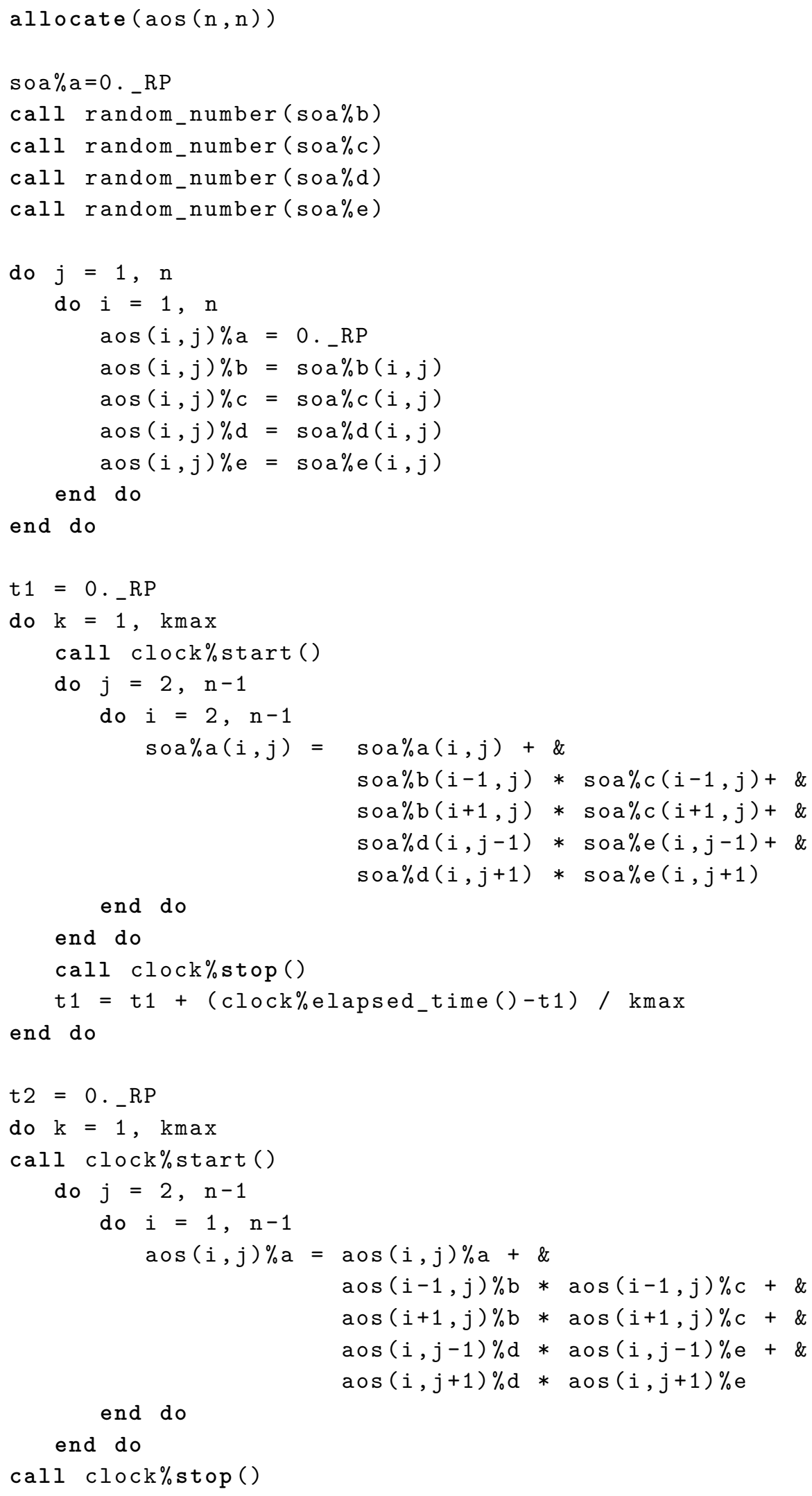




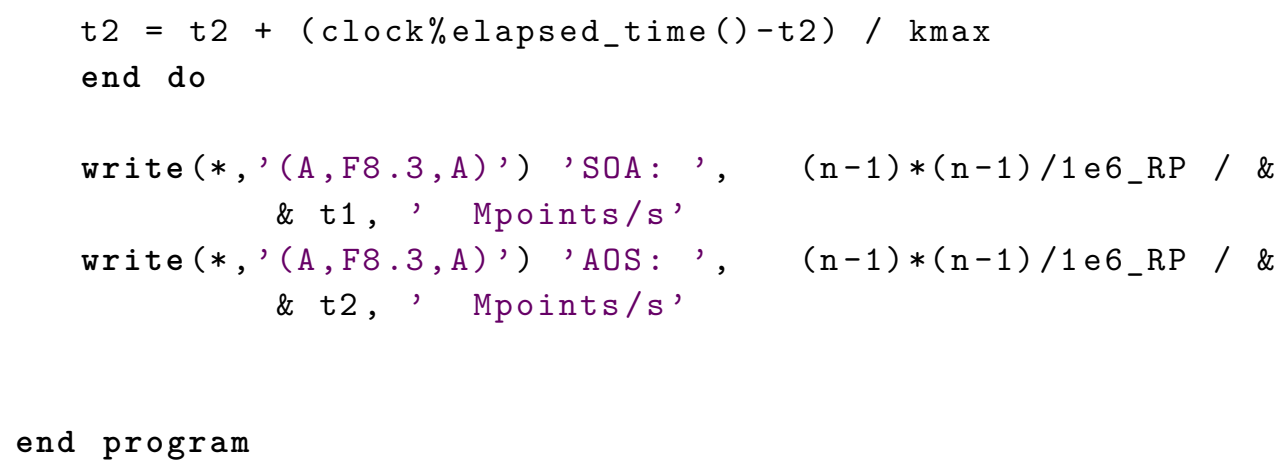

Listing A.1: SOA vs AOS timming code

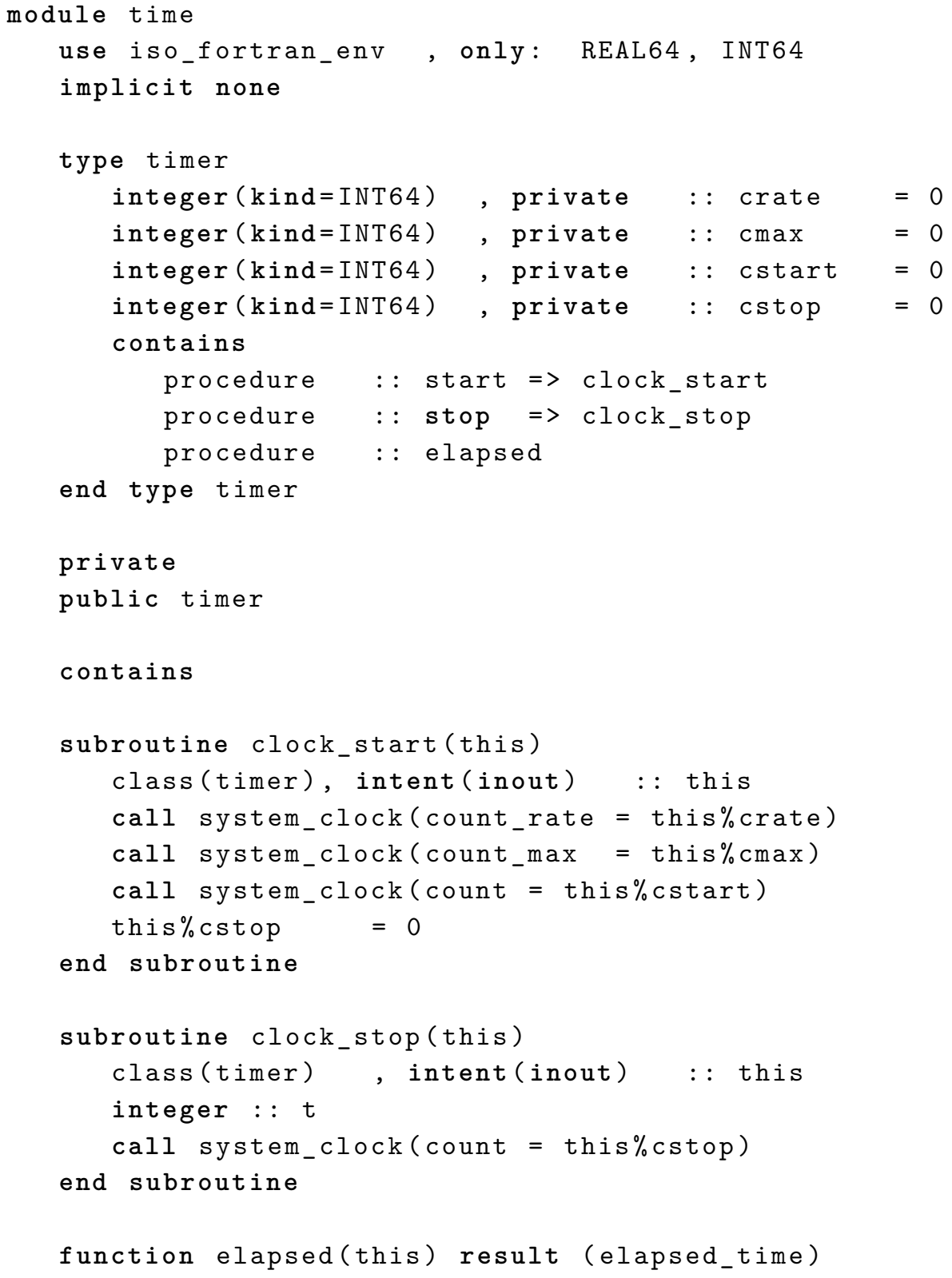




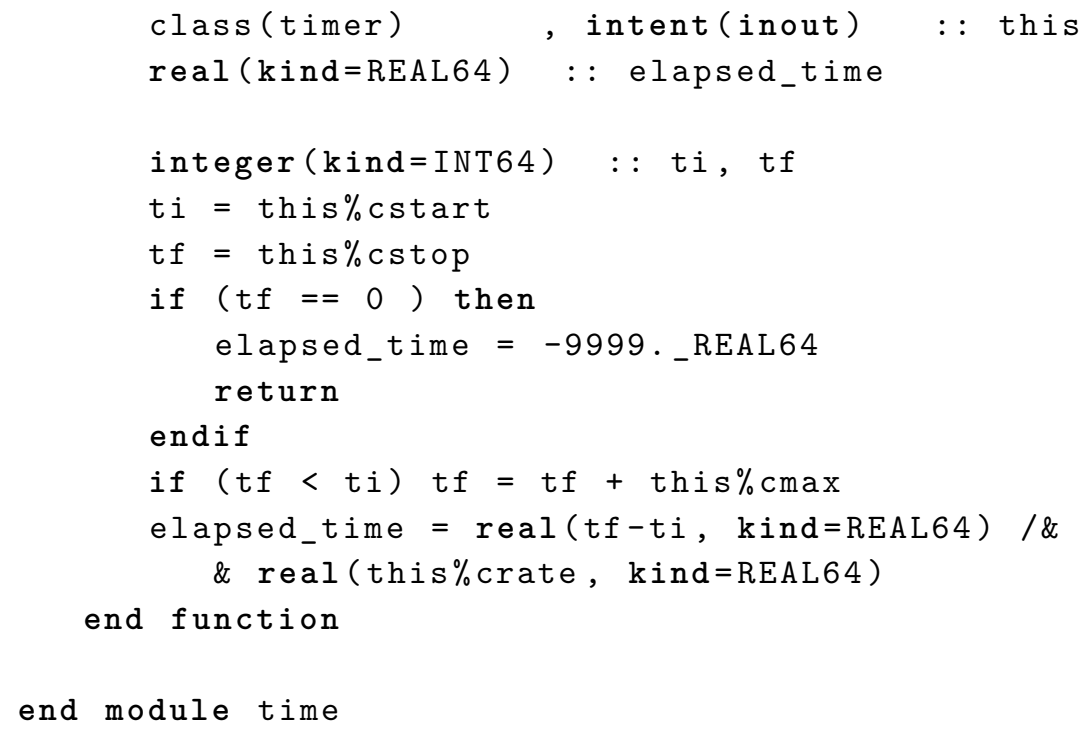

Listing A.2: time.f90

The flag -xavx tells the compiler to use, when possible, vector instructions, -parallel activates the automatic multi-thread paralellisation and -qopt-report=2 generates a compilation report. The report indicates that the main loops at lines 65 and 81 are parallelised and vectorized automatically by the compiler.

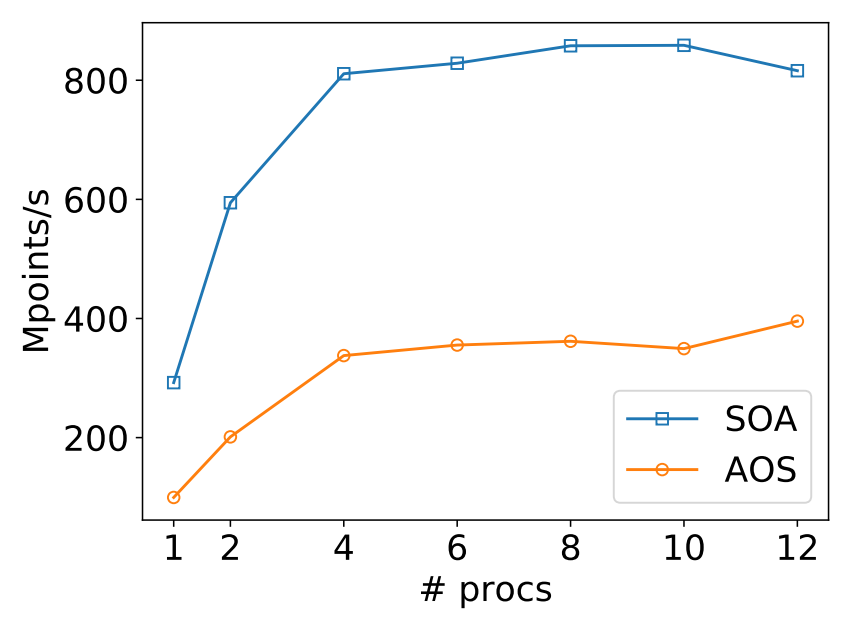

Figure A.1: Scalability of point updates per second for the two different approaches for the multiplication loop. On y-axis, million of point updates per second.

The performance results for each strategy, AOS and SOA are plotted in Figure A.1. As can be observed, the performance of the SOA strategy outperforms by a factor of 3 the AOS strategy for this kind of loops. It can be noticed as well, that the memory bandwidth becomes the computation bottleneck with more than 4 processors, this 
fact has been checked using Intel VTune profiler that indicate that the main loops are memory bounded with more than 4 processors. In Figure A. 2 the memory bandwidth from the profile is plotted. It is observed that the SOA strategy needs less memory write operations due to a better cache utilization, increasing the effective memory throughput.

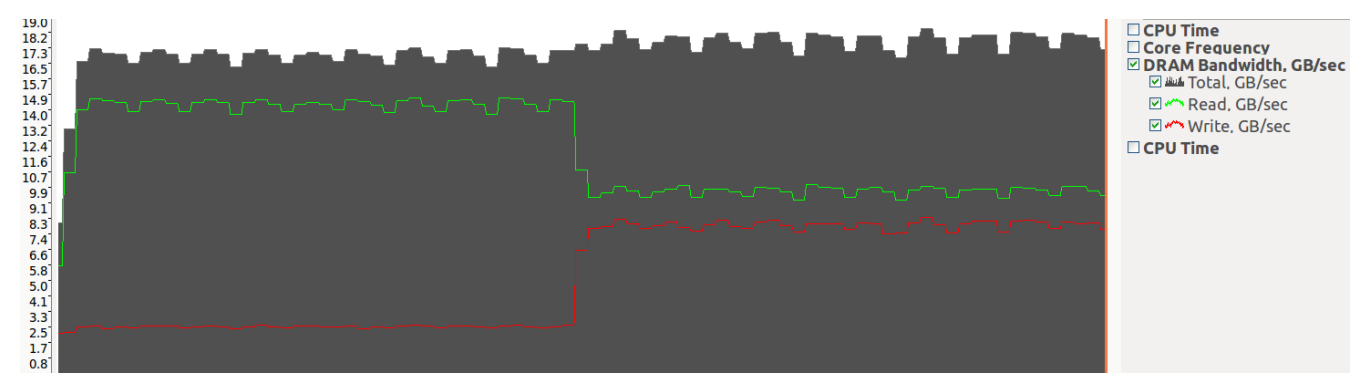

Figure A.2: Memory bandwidth utilization during test code execution Green line corresponds to read operations and red line to write operations. Left half correspond to the SOA loop and right half to the AOS loop. Profiled with Intel Vtune Amplifier XE 2017.

Another kind of loops with high arithmetic cost per computation are also present in the algorithm. In these loops memory bandwidth is not the limiting resource due to a more efficient cache use. For measuring the efficiency os this kind of loops, the multiplication operation in the main loops (lines 65 and 81) is substituted by an exponentiation ( $\sim 25$ times more costly). In Figure A.3 the scalability with the exponentiation operation is shown. The number of updates per second is reduced due to the extra cost of the loop operation but the scalability is near ideal for both strategies and no signs of memory bounding are appreciated.

Based on the results shown in this chapter, the SOA strategy has been chosen because of the better performance shown in both light and heavy arithmetic loops. 


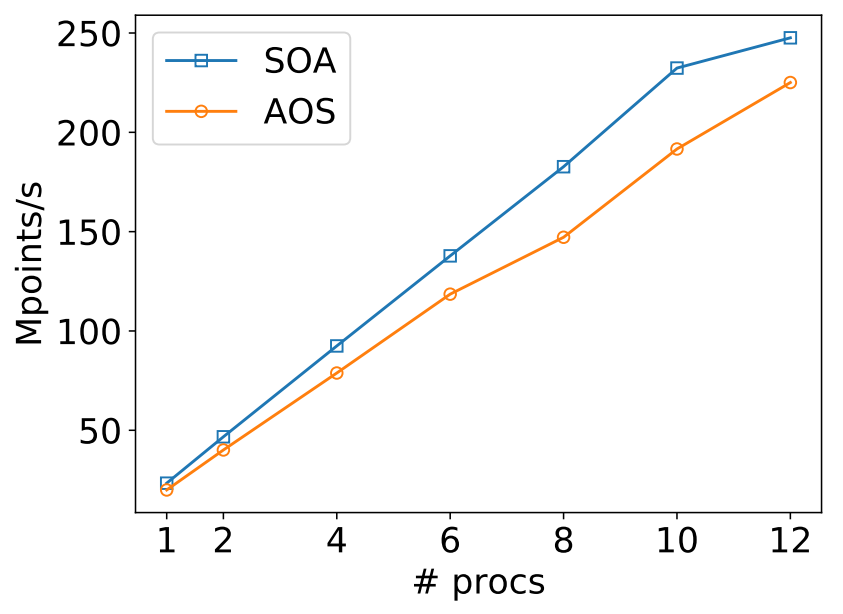

Figure A.3: Scalability of point updates per second for the two different approaches for the exponentiation loop. 



\section{appendix $B$}

\section{FORTRAN - PYTHON PLOTTING}

Fortran lacks of native utilities for graphical data representation. For this purpose, python and Matplotlib [Hunter, 2007] have been used. Matplotlib is a flexible, highly configurable python library that produces high quality figures for publication in multiple formats as well as configurable and interactive screen plots.

Data for plotting is written by Fortran code using stream unformatted files, included in the Fortran 2003 standard. Stream files do not include any records termination (which, depending on the platform, may be line-feeds, or carriage-returns, or both). These files can be read easily from python using numpy [van der Walt et al., 2011]. A minimal working example for writing-reading-plotting is shown below. In Code B.1 the Fortran side of the interface is listed. The python side of the code is shown in Code B.2.

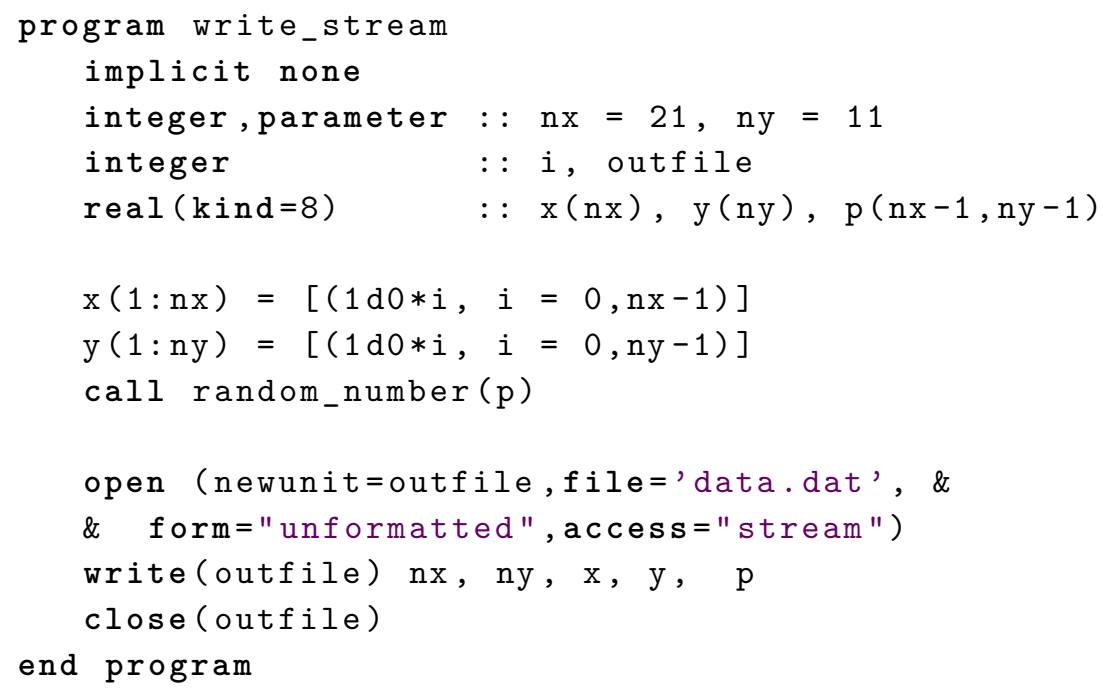

Code B.1: Minimal working example of stream writting in fortran. 


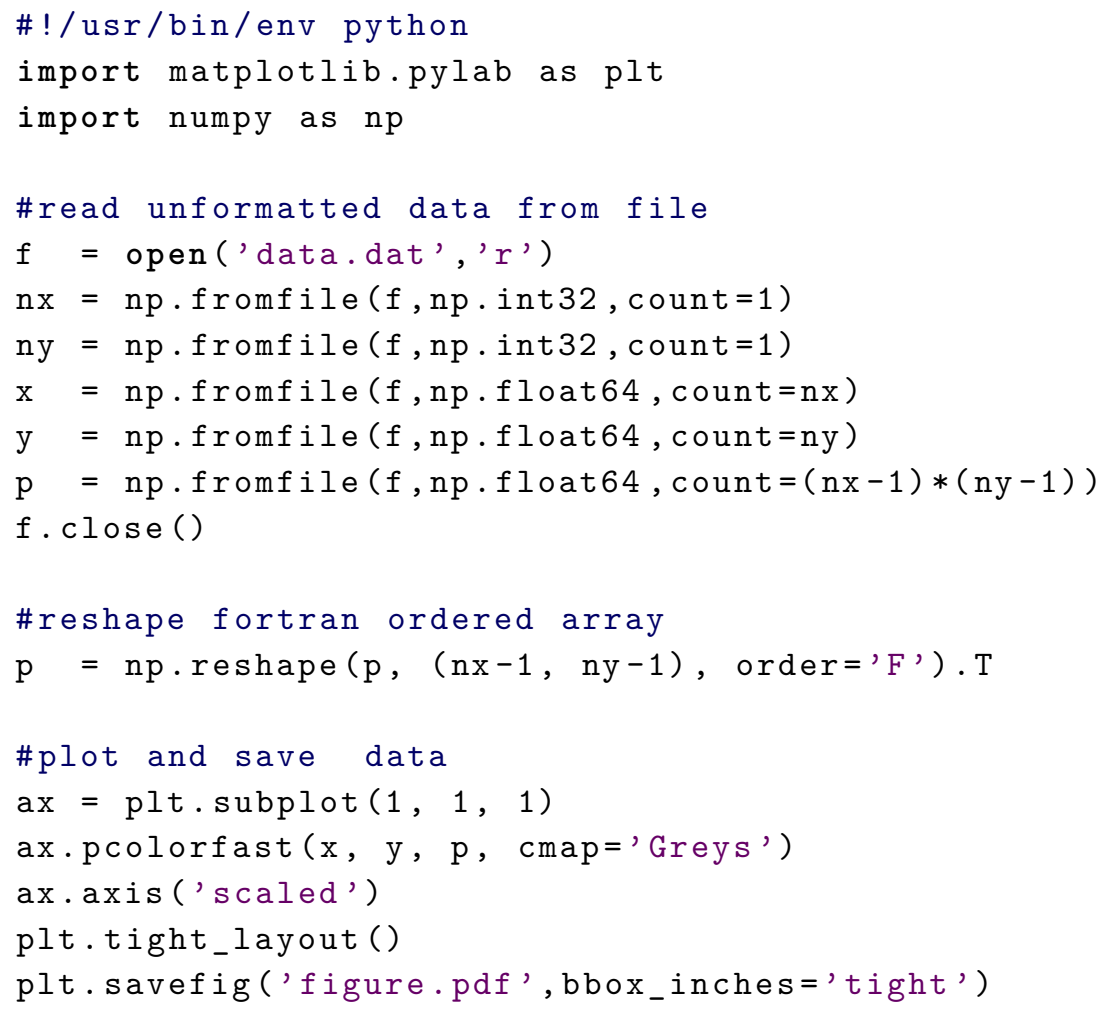

Code B.2: Minimal working example of python code for reading and plotting Fortran stream data.

This Fortran-python interface for plotting has been used for generating the majority of the plots in this work. Plots can be generated directly from the Fortran program using a system call to execute the python script with:

call execute_command_line('path/to/python/plotter').

The example code listed in this appendix generates a 2D random field on a cartesian cell centered grid of size $20 \times 10$. The plot generated can be seen in Figure B.1.

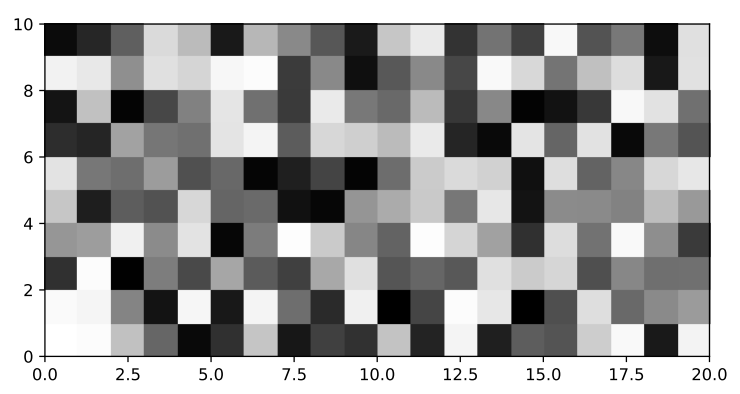

Figure B.1: Figure generated with the Fortran-Python code listed in this section. 


\section{BIBLIOGRAPHY}

[Aarnes et al., 2007] Aarnes, J. E., Gimse, T., and Lie, K.-A. (2007). An introduction to the numerics of flow in porous media using Matlab, pages 265-306. Springer Berlin Heidelberg, Berlin, Heidelberg.

[Amarnath, 1999] Amarnath, A. (1999). Enhanced Oil Recovery Scoping Study. Electric Power Research Institute (EPRI).

[Amestoy et al., 2001] Amestoy, P. R., Duff, I. S., Koster, J., and L'Excellent, J.-Y. (2001). A fully asynchronous multifrontal solver using distributed dynamic scheduling. SIAM Journal on Matrix Analysis and Applications, 23(1):15-41.

[Balay et al., 2016] Balay, S., Abhyankar, S., Adams, M. F., Brown, J., Brune, P., Buschelman, K., Dalcin, L., Eijkhout, V., Gropp, W. D., Kaushik, D., Knepley, M. G., McInnes, L. C., Rupp, K., Smith, B. F., Zampini, S., Zhang, H., and Zhang, H. (2016). Petsc web page. http://www.mcs.anl.gov/petsc.

[Barrett et al., 1994] Barrett, R., Berry, M., Chan, T. F., Demmel, J., Donato, J., Dongarra, J., Eijkhout, V., Pozo, R., Romine, C., and der Vorst, H. V. (1994). Templates for the solution of linear systems: building blocks for iterative methods, 2nd edition. SIAM, Philadelphia, PA.

[Bear, 1972] Bear, J. (1972). Dynamics of fluids in porous media. Dover Civil and Mechanical Engineering Series. Dover.

[Bear and Bachmat, 1990] Bear, J. and Bachmat, Y. (1990). Introduction to modeling of transport phenomena in porous media. Theory and Applications of Transport in Porous Media. Springer Netherlands, 1 edition.

[Boek and Venturoli, 2010] Boek, E. S. and Venturoli, M. (2010). Lattice-boltzmann studies of fluid flow in porous media with realistic rock geometries. Computers \& Mathematics with Applications, 59(7):2305-2314. 
[Bowers and Katsube, 2002] Bowers, G. and Katsube, T. (2002). The role of shale pore structure on the sensitivity of wire-line logs to overpressure. AAPG Memor, 76:43-60.

[Brekke et al., 1993] Brekke, K., Johansen, T., and Olufsen, R. (1993). A new modular approach to comprehensive simulation of horizontal wells. In SPE Annual Technical Conference and Exhibition, Houston, Texas. Society of Petroleum Engineers.

[Brooks and Corey, 1964] Brooks, R. and Corey, A. (1964). Hydraulic properties of porous media. Colorado State University Hydrology Papers. Colorado State University.

[Brown and Walker, 1997] Brown, P. N. and Walker, H. F. (1997). GMRES on (nearly) singular systems. SIAM J. Matrix Anal. Appl., 18(1):37-51.

[Buckley and Leverett, 1942] Buckley, S. E. and Leverett, M. C. (1942). Mechanism of fluid displacement in sands. Trans, AIME, 146(1):107-116.

[Cense and Berg, 2009] Cense, A. W. and Berg, S. (2009). The vicous-capillary parados in 2-phase flow in porous media. In International Symposium of the Society of Core Analysts.

[Chen, 2001] Chen, Z. (2001). Degenerate two-phase incompressible flow. Journal of Differential Equations, 171(2):203 - 232.

[Chen et al., 2004] Chen, Z., Huan, G., and Li, B. (2004). An improved impes method for two-phase flow in porous media. Transport in Porous Media, 54(3):361-376.

[Christie and Blunt, 2001] Christie, M. and Blunt, M. (2001). Tenth spe comparative solution project: a comparison of upscaling techniques. In SPE Reservoir Simulation Symposium. Society of Petroleum Engineers.

[Coats, 2003] Coats, K. (2003). IMPES stability: the CFL limit. SPE Journal, 8(03):291-297.

[Coats et al., 1969] Coats, K. H., of Texas., U., of Engineering., C., and Institutes., E. (1969). Elements of reservoir simulation. University of Texas, [Austin, TX].

[Collins and Nghiem, 1992] Collins, D. and Nghiem, L. (1992). An efficient approach to adaptive-implicit compositional simulation with an equation of state. SPE Reservoir Engineering, 7(May):259-264. 
[da Silva et al., 2016] da Silva, R. S., Lyra, P. R. M., Willmersdorf, R. B., and de Carvalho, D. K. E. (2016). A higher resolution edge-based finite volume method for the simulation of the oil-water displacement in heterogeneous and anisotropic porous media using a modified IMPES method. International Journal for Numerical Methods in Fluids, 82(12):953-978.

[Dawson et al., 1997] Dawson, C. N., Klie, H., Wheeler, M. F., and Woodward, C. S. (1997). A parallel, implicit, cell-centered method for two-phase flow with a preconditioned Newton-Krylov solver. Computational Geosciences, 1(1997):215249.

[Ding, 2011] Ding, D. (2011). Coupled simulation of near-wellbore and reservoir models. Journal of Petroleum Science and Engineering, 76(1-2):21-36.

[Dumkwu et al., 2012] Dumkwu, F. A., Islam, A. W., and Carlson, E. S. (2012). Review of well models and assessment of their impacts on numerical reservoir simulation performance. Journal of Petroleum Science and Engineering, 8283:174-186.

[Ewing, 1983] Ewing, R. (1983). 1. problems arising in the modeling of processes for hydrocarbon recovery. In Frontiers in Applied Mathematics, pages 3-34-. Society for Industrial and Applied Mathematics.

[Franc et al., 2016] Franc, J., Horgue, P., Guibert, R., and Debenest, G. (2016). Benchmark of different CFL conditions for IMPES. Comptes Rendus Mécanique, 344(10):715 - 724.

[Hornung and Jäger, 1991] Hornung, U. and Jäger, W. (1991). Diffusion, convection, adsorption, and reaction of chemicals in porous media. Journal of Differential Equations, 92(2):199 - 225.

[Hoteit and Firoozabadi, 2008] Hoteit, H. and Firoozabadi, A. (2008). Numerical modeling of two-phase flow in heterogeneous permeable media with different capillarity pressures. Advances in Water Resources, 31(1):56-73.

[Hunter, 2007] Hunter, J. D. (2007). Matplotlib: A 2d graphics environment. Computing In Science \& Engineering, 9(3):90-95.

[Hurtado et al., 2006] Hurtado, F., Maliska, C., and Silva, A. (2006). A variable timestep strategy for accelerating the IMPES solution algorithm in reservoir simulation. Proceedings of the XXVII Iberian Latin American Congress on Computational Methods in Engineering. Belém, Brasil: UFPA, (September):14. 
[Intel Corporation, 2017] Intel Corporation (2017). Developer reference for Intel ${ }^{\circledR}$ Math Kernel Library - Fortran.

[Karlo et al., 2009] Karlo, D., Carvalho, E. D., Soares, R., Roberto, P., and Lyra, M. (2009). A 3-d edge-based higher order finite volume procedure for the simulation of two phase flow of oil and water in porous media using a modified impes. Proceedings of COBEM 2009.

[Kou and Sun, 2010a] Kou, J. and Sun, S. (2010a). A new treatment of capillarity to improve the stability of impes two-phase flow formulation. Computers \& Fluids, 39(10):1923 - 1931.

[Kou and Sun, 2010b] Kou, J. and Sun, S. (2010b). On iterative IMPES formulation for two phase flow with capillarity in heterogeneous porous media. International Journal of Numerical Analysis and ..., 1(1):20-40.

[Kuzmin, 2006] Kuzmin, D. (2006). On the design of general-purpose flux limiters for finite element schemes. i. scalar convection. Journal of Computational Physics, 219(2):513 - 531.

[Künze et al., 2013] Künze, R., Lunati, I., and Lee, S. H. (2013). A multilevel multiscale finite-volume method. Journal of Computational Physics, 255:502-520.

[Lee et al., 2011] Lee, T., Leok, M., and McClamroch, N. H. (2011). Geometric numerical integration for complex dynamics of tethered spacecraft. Proceedings of the 2011 American Control Conference, (March):1885-1891.

[Leeftink et al., 2015] Leeftink, T., Latooij, C., and Rossen, W. (2015). Injectivity errors in simulation of foam eor. Journal of Petroleum Science and Engineering, 126:26-34.

[Leverett, 1941] Leverett (1941). Capillary behavior in porous solids. Trans, AIME, (142):159-172.

[Liu et al., 2016] Liu, H., Wang, K., Chen, Z., Luo, J., Deng, H., and He, Y. (2016). Numerical simulation of multi-phase flow in porous media on parallel computers.

[Mcdowell et al., 2016] Mcdowell, A., Zarrouk, S. J., and Clarke, R. (2016). Modelling viscous fingering during reinjection in geothermal reservoirs. Geothermics, 64:220-234. 
[Muccino et al., 1998] Muccino, J. C., Gray, W. G., and Ferrand, L. A. (1998). Toward an improved understanding of multiphase flow in porous media. Reviews of Geophysics, 36(3):401-422.

[Nessyahu and Tadmor, 1990] Nessyahu, H. and Tadmor, E. (1990). Non-oscillatory central differencing for hyperbolic conservation laws. Journal of Computational Physics, 87(2):408 - 463.

[Oberkampf and Roy, 2010] Oberkampf, W. L. and Roy, C. J. (2010). Verification and validation in scientific computing. Cambridge University Press, New York, NY, USA, 1st edition.

[Panfilov et al., 2012] Panfilov, M., Ghesmoune, M., and Abadpour, A. (2012). Method of negative saturation and interface stabilization for multiphase compositional flow in porous media. In ECMOR XIII - 13th European Conference on the Mathematics of Oil Recovery.

[Pavone, 1992] Pavone, D. (1992). Observations and correlations for immiscible viscous-fingering experiments. SPE Reservoir Engineering, 7(2):187-194.

[Peaceman, 1978] Peaceman, D. (1978). Interpretation of well-block pressures in numerical reservoir simulation. Society of Petroleum Engineers Journal, 18(3):183-194.

[Pourafshary et al., 2009] Pourafshary, P., Varavei, A., Sepehrnoori, K., and Podio, A. (2009). A compositional wellbore/reservoir simulator to model multiphase flow and temperature distribution. Journal of Petroleum Science and Engineering, 69(1-2):40-52.

[Riaz and Meiburg, 2003] Riaz, A. and Meiburg, E. (2003). Three-dimensional miscible displacement simulations in homogeneous porous media with gravity override. Journal of Fluid Mechanics, 494:95-117.

[Saad, 2003] Saad, Y. (2003). Iterative Methods for Sparse Linear Systems: Second Edition. Society for Industrial and Applied Mathematics.

[Saad and Schultz, 1986] Saad, Y. and Schultz, M. H. (1986). GMRES: A generalized minimal residual algorithm for solving nonsymmetric linear systems. SIAM J. Sci. Stat. Comput., 7(3):856-869.

[Sajjadi and Azaiez, 2012] Sajjadi, M. and Azaiez, J. (2012). Thermo-viscous fingering in heterogeneous media. In SPE Heavy Oil Conference Canada. Society of Petroleum Engineers. 
[Schenk and Gärtner, 2004] Schenk, O. and Gärtner, K. (2004). Solving unsymmetric sparse systems of linear equations with pardiso. Future Generation Computer Systems, 20(3):475 - 487. Selected numerical algorithms.

[Sheldon et al., 1959] Sheldon, J. W., Zondeck, B., and Cardwell, W. J. (1959). One dimensional, incompressible, noncapillary two-phase fluid flow in porous media. Trans, AIME, 216:290-296.

[Shi et al., 2015] Shi, J., Huang, L., Li, X., and Sepehrnoori, K. (2015). Production forecasting of gas condensate well considering fluid phase behavior in the reservoir and wellbore. Journal of Natural Gas Science and Engineering, 24:279-290.

[Simmons, 2008] Simmons, C. T. (2008). Henry darcy (1803-1858): Immortalised by his scientific legacy. Hydrogeology Journal, 16(6).

[Stone and Garder Jr., 1961] Stone, H. L. and Garder Jr., A. O. (1961). Analysis of gas-cap or dissolved-gas drive reservoirs. T. SPE AIME, 1(2).

[Sun et al., 2009] Sun, P., Xue, G., Wang, C., and Xu, J. (2009). Fast numerical simulation of two-phase transport model in the cathode of a polymer electrolyte fuel cell. Commun. Comput. Phys., 6(1):49-71.

[Sweby, 1984] Sweby, P. K. (1984). High resolution schemes using flux limiters for hyperbolic conservation laws. SIAM Journal on Numerical Analysis, 21(5):9951011.

[Tan and Kalogerakis, 1991] Tan, T. B. and Kalogerakis, N. (1991). A fully implicit, three-dimensional, three-phase simulator with automatic history-matching capability. Proc. of the 11TH SPE Reservoir Simulation Symp. (Anaheim, California), pages 35-46.

[Todd et al., 1972] Todd, M., O'Dell, P., and Hirasaki, G. (1972). Methods for increased accuracy in numerical reservoir simulators. Society of Petroleum Engineers Journal, 12(6):515-530.

[van der Walt et al., 2011] van der Walt, S., Colbert, S. C., and Varoquaux, G. (2011). The NumPy array: A structure for efficient numerical computation. Computing in Science Engineering, 13(2):22-30.

[Van Genuchten, 1980] Van Genuchten, M. T. (1980). A closed-form equation for predicting the hydraulic conductivity of unsaturated soils. Soil science society of America journal, 44(5):892-898. 
[Webb, 2006] Webb, S. W. (2006). Gas transport mechanisms, chapter 2, pages 5-26. Springer Netherlands, Dordrecht.

[Whitaker, 1986] Whitaker, S. (1986). Flow in porous media i: A theoretical derivation of Darcy's law. Transport in Porous Media, 1(1):3-25.

[Wu and Qin, 2009] Wu, Y.-s. and Qin, G. (2009). A generalized numerical approach for modeling multiphase flow and transport in fractured porous media. Communications in Computational Physics, 6(1):85-108.

[Yang et al., 2016] Yang, H., Yang, C., and Sun, S. (2016). Active-set reduced-space methods with nonlinear elimination for two-phase flow problems in porous media. SIAM Journal on Scientific Computing, 38(4):B593-B618.

[Zeng and Grigg, 2006] Zeng, Z. and Grigg, R. (2006). A criterion for non-Darcy flow in porous media. Transport in Porous Media, 63(1):57-69. 


